\title{
Morphological description and comparison of the dental remains from Atapuerca-Sima de los Huesos site (Spain)
}

\author{
María Martinón-Torres ${ }^{\mathrm{a}, *}$, José María Bermúdez de Castro ${ }^{\mathrm{a}}$, Aida Gómez-Robles ${ }^{\mathrm{a}}$, Leyre Prado-Simón \\ Juan Luis Arsuaga ${ }^{\mathrm{b}}$ \\ ${ }^{a}$ National Research Center on Human Evolution (CENIEH), Paseo Sierra de Atapuerca, s/n, 9002 Burgos, Spain \\ ${ }^{\mathrm{b}}$ Centro Mixto UCM-ISCIII de Evolución y Comportamiento Humanos, CMonforte de Lemos 5, Pabellón 14, 28029 Madrid, Spain
}

Keywords:

Atapuerca

Sima de los Huesos

Homo heidelbergensis

Homo neanderthalensis

Teeth

Dental morphology

\begin{abstract}
A B S T R A C T
The systematic excavation of the Sima de los Huesos (SH) site in Sierra de Atapuerca (Burgos, Spain) has yielded the largest hominin collection worldwide for the Middle Pleistocene. The dental sample now consists of more than 500 teeth that provide exceptional opportunities to define the dental morphological pattern of a Middle Pleistocene population as well as develop hypotheses about the origins of the Neanderthals. The dental collection has now increased to over 533 specimens ( 525 permanent and 8 deciduous teeth), necessitating new morphological assessments. Thus, we present a detailed morphological description of the SH permanent dentition recovered up to 2007, accomplishing comparisons with European Middle Pleistocene hominins, Neanderthals, and early and contemporary Homo sapiens. We find that SH dentitions present all the morphological traits that, either in their degree of expression, frequency, or particular combination, are usually considered as typical of Homo neanderthalensis. This study ratifies the deep roots of the Neanderthal lineage in the Middle Pleistocene of Europe. In addition, SH teeth are morphologically "more Neanderthal" than other penecontemporaneous Middle Pleistocene samples such as Mauer or Arago, and even more derived than some classic Neanderthal samples. Thus, our study would not sustain the linearity of the accretion process hypothesized for the origins of the Neanderthals, and we suggest that other evolutionary models and scenarios should be explored for the Middle and Upper Pleistocene of Europe. We propose that more than one hominin lineage may have coexisted during the Middle Pleistocene in Europe.
\end{abstract}

\section{Introduction}

Publications on hominin dental samples from Sima de los Huesos (Sierra de Atapuerca, Spain) during the last two decades have been abundant and diverse, addressing a varied range of topics such as systematics and phylogeny (e.g., Bermúdez de Castro, 1986, 1987, 1988, 1993; Bermúdez de Castro et al., 2003a, 2004a, 2009; Martinón-Torres, 2006; Martinón-Torres et al., 2006, 2007a,b; Gómez-Robles et al., 2007, 2008), sexual dimorphism (e.g., Bermúdez de Castro et al., 1993, 2001; Rosas et al., 2002), paleoecology (e.g., Bermúdez de Castro et al., 1988, 2003b; PérezPérez et al., 2003; Lozano-Ruiz et al., 2004; Lozano et al., 2008), paleodiet (Pérez-Pérez et al., 1999), paleodemography (Bermúdez de Castro and Nicolás, 1997; Bermúdez de Castro et al., 2004b,c), pathology (Bermúdez de Castro and Díez, 1995; Bermúdez de
Castro and Pérez, 1995; Cunha et al., 2004), and development (Bermúdez de Castro and Nicolás, 1995; Bermúdez de Castro and Rosas, 2001; Bermúdez de Castro et al., 2003c). However, since the last detailed morphological study was carried out (Bermúdez de Castro, 1993), the number of dental specimens that have been discovered has increased from 133 to 525, mandating new assessments of these samples and promoting tests of new hypotheses regarding human evolution. Because variation in dental form is highly heritable, paleoanthropologists consider teeth the "safe box" of the genetic code; thus, teeth may be more useful than other skeletal elements for assessing the affinities of extant and extinct human populations (e.g., Turner, 1969; Irish, 1993; Irish and Guatelli-Steinberg, 2003; Martinón-Torres et al., 2007b).

The Atapuerca-Sima de los Huesos site is a small cavity of $8 \mathrm{~m}^{2} \times 4 \mathrm{~m}^{2}$ that belongs to the Cueva Mayor-Cueva del Silo karst system, from which $80 \%$ of the entire Middle Pleistocene Homo fossil record has been recorded. To date, the human fossil assemblage recovered from this locality comprises approximately 6500 remains assigned to at least 28 separate individuals (Bermúdez de 
Castro et al., 2004a,b,c). Previous radiometric studies (U-series) of a $14 \mathrm{~cm}$ thick in situ speleothem overlying the fossil-bearing level provided an age estimate of $>350$ ka for the SH hominins (Bischoff et al., 2003). The recent analysis of another speleothem that overlies this level provided a minimum age of $530 \mathrm{ka}$ (Bischoff et al., 2007). The presence of an archaic form of Ursus deningeri, Panthera leo cf. fossilis, and Clethrionomys acrorhiza are in agreement with these dates and would suggest that SH corresponds to Marine Isotope Stage (MIS) 15/16 (500-600 ka) (García and Arsuaga, 2010). The fact that all the fossils were deposited during the same sedimentation episode (Bischoff et al., 1997), together with their relative homogeneity, supports the hypothesis that all the individuals belong to the same biological population. This site has been put forth as the first hominin evidence of anthropic accumulation possibly associated with a symbolic ritual, which is based on the discovery of a handaxe with exceptional characteristics (Carbonell et al., 2003). Thus, the SH fossils have become the most representative sample for the Middle Pleistocene worldwide, providing an incomparable opportunity to address intra-population variability (e.g., Arsuaga et al., 1997a; Lorenzo et al., 1998; Martinón-Torres et al., 2006; Gómez-Olivencia et al., 2007; Gómez-Robles et al., 2007, 2008, 2011a; Gómez-Olivencia, 2009). In addition, this locality provides the best and possibly earliest example of a hominin population with clear Neanderthal traits (e.g., Bermúdez de Castro, 1986; Arsuaga et al., 1991, 1993, 1997b,c); and contributes extraordinary data to the debate on the origins of Homo neanderthalensis. According to Arsuaga et al. (1997c: 276), "Middle Pleistocene Europeans and Neandertals represent the same 'evolutionary' species, an ancestral-descendant sequence of populations without rupture of the reproductive continuity." In this context, SH hominins have been assigned to Homo heidelbergensis (Arsuaga et al., 1991, 1993, 1997c; Bermúdez de Castro et al., 2004b), understood as a chronospecies of the European Neanderthal lineage (Arsuaga et al., 1991, 1993, 1997c). Thus, $H$. heidelbergensis could be defined by a combination of primitive traits (plesiomorphies) not found in later Neanderthals, incipient Neanderthal-like traits (mesomorphies), and typical Neanderthal traits (apomorphies) (Arsuaga et al., 1997c). It has also been suggested that many of the so-called typical Neanderthal traits are not Neanderthal autapomorphies but primitive features that, because of their high frequencies and degrees of expression, and their distinctive combination, become typical of $H$. neanderthalensis species (e.g., Patte, 1962; Franciscus and Trinkaus, 1995; Bailey, 2002a; Martinón-Torres et al., 2006; Bailey et al., 2009; Hublin, 2009). Thus, the origin of the Neanderthal lineage would imply increasing frequencies of Neanderthal traits throughout time, favored by isolation due to glacial conditions (Arsuaga et al., 1993; Hublin, 1996, 1998; Dean et al., 1998). This is the basis for the formulation of the "accretion model," in which Neanderthal distinctions would accumulate gradually in populations (Dean et al., 1998; Hublin, 1998), so that earlier Middle Pleistocene populations should be "less Neanderthal" than later populations. However, the present chronological and fossil evidence indicates that the Neanderthal morphological pattern may not have appeared quite as progressively and ordered, as has been suggested, and therefore other models should also be explored (Bermúdez de Castro et al., 2009; Dennell et al., 2010). In this context, we present a detailed and updated description and comparison of the Sima de los Huesos dental sample, and we explore their degree of Neanderthal affinity by comparing them to Homo sapiens, $H$. neanderthalensis, and other Middle Pleistocene samples from Europe.

The present study focuses on the morphology of the permanent dentition. Previous studies have revealed that the SH hominins showed a remarkable imbalance between the size of their lower and upper anterior (Il-P3) and posterior (P4-M3) teeth in relation to other Pleistocene hominins and modern humans (Bermúdez de Castro, 1993). These studies have also pointed out that SH hominins had mandibular posterior teeth that were smaller in absolute terms than those of Plio-Pleistocene hominins (Bermúdez de Castro, 1986) and were comparable in size to those of modern humans, apart from other traits such as similar intermolar size ratios and the frequent absence of a hypoconulid (Bermúdez de Castro and Nicolás, 1995; Gómez-Robles, 2010; Gómez-Robles et al., 2011a). This pattern of dental reduction could be related to the absolutely and relatively delayed development of posterior teeth in later Homo species. The decrease of the rate of cell proliferation, which affected the later-forming crown regions to a greater extent, may be the biological process responsible for the general and differential dental size reduction in this group, and thus, it might reflect ontogenetic changes (Bermúdez de Castro and Nicolás, 1995; Bermúdez de Castro et al., 2003c; Bermúdez de Castro and Rosas, 2001). As a result of the large sample size now available, and because our morphological comparison focuses on taxonomic and phylogenetic factors, wehave decided to present metric comparisons in a separate paper with special attention to the biological processes that were responsible for the dental reduction. Consequently, ref erences to the size of molar cusps in this paper are based on qualitative ranked scales (see Methods Section), and not the measures themselves. Still, the buccolingual (BL) and mesiodistal (MD) diameters of each tooth and the mean and standard deviation for each tooth class for the Sima de los Huesos sample are also provided, although they will not be compared.

\section{Materials}

\section{The $\mathrm{SH}$ dental sample}

The total dental sample from the SH site up to 2007 comprises 525 permanent and 8 deciduous teeth (Table 1, SOM Table 1). Since the last detailed morphological study conducted in 1993 (Bermúdez de Castro, 1993), the dental collection has increased from 133 to 533 specimens, and, therefore, a new morphological assessment was deemed necessary.

Analyses of maxillae, mandibles, and isolated teeth provide the best method for obtaining the minimum number of individuals (MNI) represented in the sample. We considered the fit of isolated teeth in their corresponding alveoli, tooth size, morphology (antimeres), wear, the nature of the interproximal and occlusal wear facets, and matching hypoplastic enamel defects with alterations in the perikymata spacing in other teeth from the same individual. The evaluation of MNI has been ongoing since the first excavation season (Bermúdez de Castro, 1986; Bermúdez de Castro and Nicolás, 1997). With this increased hypodigm, our latest assessment is that the MNI is 28 (Bermúdez de Castro et al., 2004a), leaving 101 isolated teeth unassigned. With this conservative assessment, the individual formerly identified as XXXI is now designated as individual XXVIII (Table 2).

Table 1

Summary table of the Sima de los Huesos dental sample by tooth class.

\begin{tabular}{cc}
\hline Maxillary $(\mathrm{N})$ & Mandibular $(\mathrm{N})$ \\
\hline $\mathrm{I}^{1}: 31$ & $\mathrm{I}_{1}: 30$ \\
$\mathrm{I}^{2}: 28$ & $\mathrm{I}_{2}: 32$ \\
$\mathrm{C}^{\prime}: 30$ & $\mathrm{C}_{3}: 31$ \\
$\mathrm{P}^{3}: 29$ & $\mathrm{P}_{3}: 36$ \\
$\mathrm{P}^{4}: 28$ & $\mathrm{P}_{4}: 37$ \\
$\mathrm{M}^{1}: 29$ & $\mathrm{M}_{1}: 42$ \\
$\mathrm{M}^{2}: 30$ & $\mathrm{M}_{2}: 41$ \\
$\mathrm{M}^{3}: 31$ & $\mathrm{M}_{3}: 40$ \\
\hline
\end{tabular}


Table 2

Summary table of the SH individuals with their age at death, sex estimation, and preserved teeth. L: left, R: right, Md: mandibular, Mx: maxillary. Age at death estimation and sex estimation are taken from Bermîdez de Castro et al. (2004a).

\begin{tabular}{|c|c|c|c|}
\hline Individual & $\begin{array}{c}\text { Age at } \\
\text { death (yrs) }\end{array}$ & Sex & Teeth \\
\hline 1 & $16-18$ & $\mathrm{~F}$ & All Md except both $\mathrm{P}_{3}$ S. \\
\hline II & $12.5-14.5$ & - & $\begin{array}{c}\text { Md: all except both } \mathrm{M}_{3} \mathrm{~s} \text {. } \\
\mathrm{Mx} \text { : both } \mathrm{I}^{1} \mathrm{~s} \text {, both } \mathrm{I}^{2} \mathrm{~s} \text {, and } \mathrm{LC} \text {. }\end{array}$ \\
\hline III & $15-17$ & $\mathrm{~F}$ & $\begin{array}{l}\text { Md: all except } \mathrm{RI}_{1}, \mathrm{RP}_{4} \text {, and both } \mathrm{M}_{3} \mathrm{~s} \text {. } \\
\mathrm{Mx} \text { : both } \mathrm{P}^{4} \mathrm{~s} \text {, both } \mathrm{M}^{1} \mathrm{~s} \text {, and } \mathrm{LM}^{2} \text {. }\end{array}$ \\
\hline IV & $26-32$ & $\mathrm{~F}$ & $\begin{array}{l}\text { Md: all except both } \mathrm{I}_{1} \mathrm{~s} \text {, both } \mathrm{I}_{2} \mathrm{~s} \text {, } \\
\text { both } \mathrm{Cs} \text {, and } \mathrm{RM}_{1} \text {. } \\
\mathrm{Mx} \text { : all except both } \mathrm{I}^{1} \mathrm{~s} \text {, both } \mathrm{I}^{2} \mathrm{~s} \text {, } \\
\text { both } \mathrm{Cs} \text {, both } \mathrm{P}^{3} \mathrm{~s} \text {, and } \mathrm{LP}^{4} \text {. }\end{array}$ \\
\hline $\mathrm{V}$ & +35 & - & All $\mathrm{Mx}$ except $\mathrm{RI}^{1}, \mathrm{RI}^{2}$, and LC. \\
\hline VI & $16-18$ & $\mathrm{~F}$ & Md: both $\mathrm{P}_{3} \mathrm{~s}, \mathrm{LP}_{4}, \mathrm{LM}_{1}, \mathrm{LM}_{2}$, and $\mathrm{LM}_{3}$. \\
\hline VII & $24-30$ & M & $\begin{array}{l}\text { Md: all except } \mathrm{LM}_{2} \text { and } \mathrm{RM}_{3} \text {. } \\
\mathrm{Mx} \text { : all except both } \mathrm{I}^{2} \mathrm{~s} \text {. }\end{array}$ \\
\hline VIII & $18-20$ & M & $\mathrm{Mx}: \mathrm{LP}^{4}, \mathrm{LM}^{1}$, and $\mathrm{LM}^{2}$ \\
\hline IX & $3-4$ & - & Md: RdC. \\
\hline $\mathrm{x}$ & $15-17$ & $\mathrm{~F}$ & $\mathrm{Md}: \mathrm{RC}, \mathrm{RP}_{4}$, both $\mathrm{M}_{1} \mathrm{~s}$, and $\mathrm{LM}_{2}$ \\
\hline $\mathrm{XI}$ & $13-15$ & $\mathrm{~F}$ & $\begin{array}{c}\text { Md: both } \mathrm{I}_{2} \mathrm{~s}, \mathrm{LC}, \mathrm{RP}_{3}, \mathrm{RP}_{4} \text {, both } \mathrm{M}_{1} \mathrm{~s} \text {, } \\
\text { and both } \mathrm{M}_{2} \mathrm{~s} \text {. } \\
\mathrm{Mx} \text { : } \mathrm{RC}^{\prime} \text {, both } \mathrm{P}^{3} \mathrm{~s} \text {, both } \mathrm{P}^{4} \mathrm{~s} \\
\text { both } \mathrm{M}^{1} \mathrm{~s}, \mathrm{RM}^{2} \text {, and } \mathrm{RM} 3 .\end{array}$ \\
\hline XII & $17-19$ & M & $\begin{array}{l}\text { Md: All except both } \mathrm{I}_{1} \mathrm{~S} \text { and } \mathrm{LP}_{4} \text {. } \\
\mathrm{Mx} \text { : All except } \mathrm{RI}^{1}, \mathrm{RP}^{4} \text {, and } \mathrm{RM}^{2} \text {. }\end{array}$ \\
\hline XIII & +35 & - & Md: $\mathrm{LM}_{2}$ \\
\hline XIV & $12.5-14.5$ & - & Md: $\mathrm{LP}_{4}$, both $\mathrm{M}_{1} \mathrm{~s}$, and both $\mathrm{M}_{2} \mathrm{~s}$. \\
\hline XV & $17-19$ & $\mathrm{~F}$ & $\begin{array}{l}\text { Md: All except } R l_{1} \text {, both } \mathrm{P}_{3} \mathrm{~S} \\
\mathrm{LM}_{1}, \mathrm{LM}_{2} \text {, and } \mathrm{LM}_{3} \text {. }\end{array}$ \\
\hline XVI & $12.5-14.5$ & - & $\begin{array}{l}\mathrm{Md} \text { : All except } \mathrm{Rl}_{1}, \mathrm{LI}_{2}, \mathrm{LM}_{2} \text {, and } \mathrm{LM}_{3} \text {. } \\
\mathrm{Mx} \text { : All except } \mathrm{RC}, \mathrm{LM}^{1} \text {, and } \mathrm{RM}^{3} \text {. }\end{array}$ \\
\hline XVII & $20-25$ & - & $\mathrm{Mx}: \mathrm{RP}^{3}, \mathrm{RP}^{4}, \mathrm{RM}^{1}$, and $\mathrm{RM}^{2}$ \\
\hline XVIII & $9.5-11.5$ & M & $\begin{array}{l}\text { Md: All plus } \mathrm{Rm}_{2} \text {. } \\
\text { Mx: All plus both } \mathrm{m}^{2} \mathrm{~s} \text {. }\end{array}$ \\
\hline XIX & $16-18$ & $\mathrm{~F}$ & $\begin{array}{l}\text { Md: } \mathrm{LM}_{1} \text {, both } \mathrm{M}_{2} \mathrm{~s} \text {, and both } \mathrm{M}_{3} \mathrm{~S} \text {. } \\
\mathrm{Mx}: \mathrm{RM}^{1} \text {, both } \mathrm{M}^{2} \mathrm{~s} \text {, and both } \mathrm{LM}^{3} \mathrm{~s} \text {. }\end{array}$ \\
\hline$x x$ & $12.5-14.5$ & M & $\begin{array}{l}\text { Md: All except } \mathrm{Rl}_{1}, \mathrm{LI}_{2} \text {, and both } \mathrm{M}_{3} \mathrm{~s} \text {. } \\
\mathrm{Mx} \text { : All except } \mathrm{RP}^{4} \text { and both } \mathrm{M}^{3} \mathrm{~s} \text {. }\end{array}$ \\
\hline XXI & +35 & M & $\begin{array}{l}\text { Md: All except both } \mathrm{I}_{1} \mathrm{~s} \text {, both } \mathrm{I}_{2} \mathrm{~s} \text {, } \\
\text { and both } \mathrm{Cs} \text {. }\end{array}$ \\
\hline XXII & $20-26$ & M & $\begin{array}{l}\text { Mx: All except both } \mathrm{I}^{2} \mathrm{~s} \text { and both } \mathrm{Cs} \text {. } \\
\text { Md: All except LC. } \\
\text { Mx: All except both } \mathrm{RI}^{1} \mathrm{~s} \text { and LC. }\end{array}$ \\
\hline XXIII & $14-16$ & $\mathrm{~F}$ & Md: All except $\mathrm{LI}_{1}$ \\
\hline XXIV & $12.5-14.5$ & - & $\begin{array}{l}\text { Md: All except } \mathrm{LI}_{1}, \mathrm{RC} \text {, and both } \mathrm{P}_{4} \mathrm{~S} \text {. } \\
\mathrm{Mx} \text { : RC and } \mathrm{RM}^{1} \text {. }\end{array}$ \\
\hline$X X V$ & $11-13$ & $\mathrm{~F}$ & Md: All except $\mathrm{LM}_{2}, \mathrm{LM}_{3}$, plus both $\mathrm{m}_{2} \mathrm{~s}$. \\
\hline XXVI & $16-18$ & $\mathrm{~F}$ & $\begin{array}{l}\text { Md: both } M_{1} s \text {, both } M_{2} s \text {, and } R_{3} \text {. } \\
M x: P^{3} \text {, both } M^{1} s \text {, both } M^{2} s \\
\text { and both } M^{3} s \text {. }\end{array}$ \\
\hline XXVII & $20-26$ & M & $\begin{array}{l}\text { Md: All except both } \mathrm{I}_{1} \mathrm{~s} \text {, both } \mathrm{I}_{2} \mathrm{~S} \text {, } \\
\text { and both } \mathrm{Cs} \text {. }\end{array}$ \\
\hline XXVIII & $24-30$ & $\mathrm{~F}$ & $\begin{array}{l}\mathrm{Md} \text { : All except } \mathrm{RI}_{2}, \mathrm{RC}, \mathrm{LP}_{3} \text {, and both } \mathrm{P}_{4} \mathrm{~s} \text {. } \\
\mathrm{Mx} \text { : Both } \mathrm{I}^{1} \mathrm{~s}, \mathrm{LI}^{2} \text {, both } \mathrm{Cs}, \mathrm{LM}^{2} \text {, and } \mathrm{LM}^{3} \text {. }\end{array}$ \\
\hline
\end{tabular}

Estimation of sex for some of these 28 individuals was performed by considering the variability of the teeth and mandible samples (Rosas et al., 2002; Bermúdez de Castro et al., 2004b,c). Estimation of the age of immature individuals was based on dental development (Bermúdez de Castro et al., 2004b,c), and we employed methods based on rates of tooth-wear in our estimation of age at death for the adult individuals (Bermúdez de Castro et al., 2003b, 2004b,c).

When teeth are in situ they retain the inventory number of the maxilla/mandible to which they belong. When mandibles are composed of more than one fragment, teeth may possess the inventory number of each of the fragments. However, the majority of the teeth have been assigned their own inventory number because they were initially recovered as isolated teeth, even though they were later assigned to their respective mandibular and maxillary specimens (SOM Table 1). The association of isolated teeth with their respective mandible/maxilla was performed by JMBC based on how well they fit into alveolar sockets, correspondence with the interproximal contact facets of adjacent teeth, and symmetry between antimeres.

\section{Comparative sample}

To explore the affinity of individuals in this sample with Neanderthals, the Sima de los Huesos teeth were compared to other hominin fossils from the Middle Pleistocene of Europe (H. heidelbergensis), a wide sample of Neanderthal fossils ( $H$. neanderthalensis), and a $H$. sapiens collection including a contemporary sample and several early, anatomically modern human specimens (fossil $H$. sapiens). The taxon $H$. heidelbergensis was used to refer to the European Middle Pleistocene populations. The separation between $H$. neanderthalensis and $H$. heidelbergensis was based on chronological criteria, assigning to $H$. neanderthalensis those specimens belonging to the Upper Pleistocene. This comparative sample consisted mostly of original fossils, but with some high quality replicas also included (see Table 3 ).

\section{Methods}

\section{Protocol for describing samples}

Observations were made on the original specimens from Sima de los Huesos. A detailed morphological description of the dental remains by tooth class is provided as well as a general morphological comparison with other hominin groups from the Middle and Upper Pleistocene. Wear was assessed following the categories in Molnar (1971). We describe the SH sample by tooth class, providing a summary of the overall morphology of each tooth type and drawing attention to individual specimens that showed any distinctive features.

Descriptions were made based on the assumption that teeth were in their original anatomical position. The descriptive terminology employed herein derives from a number of sources including Carlsen (1987), Tobias (1991), Turner et al. (1991), Scott and Turner (1997), and Martinón-Torres et al. (2007b, 2008). The basic terminology used for features of the dental crowns and the grade-scales is provided in Table 4 .

Table 3

Comparative dental sample.

\begin{tabular}{|c|c|}
\hline Species & Specimens \\
\hline H. heidelbergensis (HEI) ${ }^{a}$ & $\begin{array}{l}\text { Arago }^{\mathrm{b}} \text {; Mauer } \\
\text { Steinheim. }\end{array}$ \\
\hline H. neanderthalensis (NEA) & $\begin{array}{l}\text { Arcy-sur-Cure } \text { (Renne, Hyène, Loup, Bison); }^{\text {La Quina 5; Malarnaud }} \text {; Petit-Puymoyen }{ }^{\mathrm{b}} \text {; } \\
\text { Pinilla del Valle (Madrid, Spain); } \\
\text { Cabezo Gordo; Engis II; Fondo Cattíe; } \\
\text { Saccopastore }{ }^{\mathrm{b}} \text {; Tabun (Tabun } \mathrm{Cl}^{\mathrm{b}} \text { ); Krapina; } \\
\text { Le Moustier 1; Monsempron; Saint-Césaire; } \\
\text { Shanidar; Sidrón }(005,008), \text { Hortus. }\end{array}$ \\
\hline $\begin{array}{l}\text { Contemporary } \\
\text { H. supiens (SAP) }\end{array}$ & $\begin{array}{l}\text { Hispanic-Muslim medieval collection of } \\
\text { San Nicolás (Murcia, Spain) } \\
\text { Mesolithic North African sample } \\
\text { Aïn Meterchem, Gambetta, Aïn Dokkara, Taforalt); } \\
\text { Mesolithic French sample }{ }^{\mathbf{b}} \text { (Téviec and Hoëdic); } \\
\text { Neolithic French sample } \text { (Avize, Dolmens }^{\mathrm{b}} \\
\text { de Bretons, Caverne de L'Homme Mort, Orrouy). }\end{array}$ \\
\hline $\begin{array}{l}\text { Fossil } \\
\text { H. supiens (FSAP) }\end{array}$ & 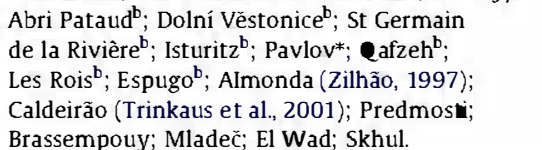 \\
\hline
\end{tabular}

a The Homo heidelbergensis denomination was employed to ref ex exclusively to the European Middle Pleistocene fossils.

b Indicates that observations were made on original fossils. 
Table 4

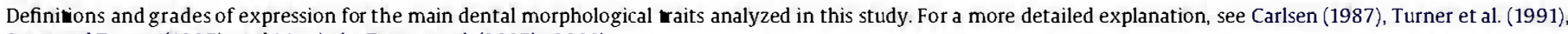
Scott and Turner (1997), and Martinón-Torres et al. (2007b, 2008).

Labial convexity: convexity of the labial surf ace when viewed from theocclusalaspect. A grade 5 was added to score labial convexities in maxillary incisors that are more pronounced than the ASU UI1 labial convexity grade 4 . This trait is scored in $\mathrm{I}^{1}, \mathrm{I}^{2}, \mathrm{I}_{1}$, and $\mathrm{I}_{2}$.

0 . Labial surface is flat

1. Labial surf ace exhibits race convexity.

2. Labial surface exhibits weak convexity.

3. Labial surface exhibits moderate convexity

4. Labial surface exhibits pronounced convexity.

5. Labial surf ace exhibits very strong convexity.

Shovel shape: presence of lingual marginal ridges and degree of expression. This rait is scored in maxillary (grades 0 to 6 ) and mandibular (grades 0 to 4 ) incisors.

0 . Marginal ridges are not expressed.

1. Faint shovel shape. The mesial and distal aspects of the lingual surface can be seen and palpated.

2. Trace of shovel shape. Elevations of the marginal ridges are easily seen.

3. Moderate shovel shape. The marginal ridges are more pronounced and there is a tendency for ridge convergence.

4. Pronounced shovel shape. The marginal ridges are clearly pronounced.

5. Strong shovel shape. There is a strong development of ridges, which almost come into contact at the cingulum

6. Marked shovel shape. Strongest development of the marginal ridges, which invade the lingual surf ace.

Tuberculum dentale: tubercles, ridges, or cusp-like features expressed in the cingular region of the lingual surface. This trait is recorded in $\mathrm{I}^{1}$ (grades 0 to 4 ),

$\mathrm{I}^{2}$ (grades 0 to 6 ), $\mathrm{C}^{\prime}$ (grades 0 to 6 ), $\mathrm{I}_{1}$ (grades 0 to 4 ), $\mathrm{I}_{2}$ (grades 0 to 4 ), and $\mathrm{C}$, (grades 0 to 4 ). Grade 0 refers to a smooth lingual surface and grade 1 in both $\mathrm{I}^{1} \mathrm{~s}$ includes those specimens with a clear basal eminence, regardless of whether or not it presents tubercles and/or crests on its surface.

0 . No expression. Cingular region of the lingual surface is smooth

1. Faint ridging.

2. Trace ridging.

3. Strong ridging

4. Pronounced ridging.

5. Weakly developed cuspule with a free apex.

6. Strong cusp with a free apex.

Canine distal accessory ridge: ridge development in the distolingual fossa, between the tooth apex and the distolingual marginal ridge. This trait is recorded in upper and lower canines.

0 . The ridge is absent

1. The ridge is weakly developed.

2. The ridge is pronounced.

Canine mesial ridge: relative development of the mesial ridge compared to the distal ridge. This trait is recorded in upper and lower canines.

0 . Mesial and distal lingual ridges are the same size. Neither is attached to the tuberculum dentale if present

1. Mesial marginal ridge is larger than the distolingual and it may be weakly attached to the tuberculum dentale.

2. Mesiolingual ridge is much larger and incorporates the tuberculum dentale. (In the upper canines this is called Morris' type.)

Buccal essential crest or ridge: degree of expression and possible bif urcation. This trait is recorded in $\mathrm{P}^{3} \mathrm{~s}$ and $\mathrm{P}^{4} \mathrm{~s}$.

0 . The crest is absent.

1. The crest is single.

2. The crest is bif urcated.

Lingual essential crest or ridge: same scoring as the buccal essential crest.

Premolar distal accessory ridge: ridge development between the tooth apex and the distolingual marginal ridge. This rait is scored in upper and lower premolars.

0 . The ridge is absent

1. The ridge is present

Premolar mesial accessory ridge: ridge development between the tooth apex and the distolingual marginal ridge. This rait is scored in upper and lower premolars.

0 . The ridge is absent.

1. The ridge is present

Transverse crest of premolars: expression of a ransverse crest connecting the main cusps of the premolars. This rait is scored in upper and lower premolars.

0 . The crest is absent. The sagittal fissure is continuous.

1. The crest is weak or it is interrupted by the sagittal fissure.

2. The crest is pronounced or the sagittal fissure is erased.

Metacone: refers to the expression of this cusp. This trait is scored in upper molars.

0 . The metacone is absent

1. An attached ridge is present at the metacone site, but there is no free apex

2. A faint cuspule with a free apex is present.

3. A weak cusp is present

4. The metacone is large.

5. The metacone is very large.

Hypocone: refers to the expression of this cusp. This trait is scored in upper molars. The grade 3.5, as specified in Turner et al. (1991), was excluded.

0 . No hypocone.

1. Faint ridging present at the site.

2. A small cuspule is present

3. A small cusp is present.

4. A large cusp is present

5. A very large cusp is present.

Cusp 5: refers to the expression of the metaconule. This trait is scored in upper molars.

0 . This cusp is absent

1. The distal groove separating the hypocone and the metacone is erased.

2. A small cuspule is present

3. A small cusp is present

4. A medium-sized cusp is present

Crist obliqua: expression of an enamel crest connecting the protocone and the metacone. This trait is scored in upper molars.

0 . The crest is absent or interrupted.

1. There is a continuous crest connecting the protocone and the metacone.

Transverse crest of molars: crest connecting the mesial aspect of the protocone and the paracone. This trait is scored in upper molars.

0 . The crest is absent or interrupted. 
1. The crest is present or continuous.

Carabelli's complex: tubercle of various degrees of expression on the lingual surface of the protocone. This trait is scored in upper molars following ASUDAS

(Turner et aI., 1991).

0 . The mesiolingual aspect of the protocone is smooth

1. A groove is present.

2. A pit is present.

3. A small Y-shaped depression is present

4. A large Y-shaped depression is present.

5. A small cusp without a free apex is present.

6. A medium-sized cusp with an attached apex making contact with the medial lingual groove is present.

7. A large free cusp is present

Parastyle: tubercle of various degrees of expression on the buccal surface of the paracone. This trait is scored in upper molars following ASUDAS (Turner et aI., 1991).

0 . The buccal surface of the paracone and metacone is smooth.

1. A pit is present in or near the buccal groove between the paracone and metacone.

2. A small cusp with an attached apex is present.

3. A medium-sized cusp with a free apex is present.

4. A large cusp with a free apex is present

5. A very large cusp with a free apex is present.

6. An effectively free peg-shaped crown attached to the root of the third molar is present.

Mesial marginal accessory tubercles: presence of tubercles in the mesial marginal ridge of upper molars. Their expression is easily affected by wear.

0 . Tubercles are absent.

1. Tubercles are present.

Lingual cusp variation of the lower premolars: refers to the number of lingual cusps, including main and accessory lingual cusps. Frequently, accessory cusps adopt

the shape of tubercles without a clear tip and are difficult to differentiate from enamel wrinkles.

0 . No lingual cusp. A ridge may be present without a free tip.

1. One lingual cusp. It shows a free apex.

2. One lingual cusp, and a ridge or platform without a free apex, separated by a small fissure.

3. Two lingual cusps.

4. Three lingual cusps.

5. More than three lingual cusps.

Anterior fovea: refers to the expression of a fovea or groove on the anterior occlusal surf ace, distal to the mesial marginal ridge. This trait is scored in lower molars.

0 . Absence of an anterior fovea.

1. Slight linear depression in the mesial marginal complex.

2. Pronounced linear or pit-like depression in the mesial marginal complex.

Middle trigonid crest: refers to the expression of a crest connecting the mesial aspects of the protoconid and the metaconid.

0 . The crest is absent

1. There is a weak crest but it is interrupted by the central groove.

2. There is a continuous bridge-like crest connecting the mesial aspects of the protoconid and the metaconid.

Distal trigonid crest: refers to the expression of a crest connecting the distal aspect of the protoconid and the metaconid.

0 . The crest is absent.

1. The crest is weak or interrupted by the central groove. It may be identified by the expression of a short fissure in the protoconid, parallel to the buccolingual fissure.

2. The crest is conspicuous and/or continuous. If it is interrupted, it may be identified by the expression of a short fissure that crosses both the protoconid and the metaconid and is parallel to the buccolingual fissure.

Deflecting wrinkle: the essential crest of the paraconid is distally deflected. This rait is scored in lower molars.

0 . The paraconid crest is straight.

1. The paraconid crest shows a midpoint constriction or is slightly deflected, but it does not make contact with the hypoconid.

2. The paraconid crest is deflected distally forming an L-shaped ridge and contacts the hypoconid.

Hypoconulid size: refers to the size of the hypoconulid or the $\mathrm{C} 5$ in lower molars.

0 . No occurrence of a hypoconulid.

1. The hypoconulid is present and very small. It may be identified by a distal bif urcation of the central fissure.

2. The hypoconulid is small.

3. The hypoconulid is medium-sized.

4. The hypoconulid is large.

5. The hypoconulid is very large.

C6 (entoconulid or tuberculum sextum): the presence of a hypoconulid is not necessarily required to record a C6. When there is one single distal cusp and it occupies a lingual position, we consider it a C6.

0 . The $\mathrm{C6}$ is absent.

1. The $\mathrm{C} 6$ has the aspect of an enamel wrinkle. This grade also covers the expression of a deep distal fovea in distolingual position.

2. It has a moderate size and it is delimitated by two grooves.

3. The $C 6$ is large.

$C 7$ (met aconulid or tuberculum intermedium): this accessory cusp develops between the metaconid and the hypoconid.

0 . The $\mathrm{C} 7$ is absent.

1. A faint, tipless $\mathrm{C7}$ is present. It may be identified by the bif urcation of the buccolingual fissure.

2. The $\mathrm{C} 7$ is small.

3. The $\mathrm{C7}$ is medium-sized.

4. The $\mathrm{C7}$ is large.

Groove pattem: This trait is scored in lower molars.

1. A Y-pattern is present ( $\mathrm{C} 2$ and $\mathrm{C} 3$ are in contact).

2. A groove pattern different from the Y-pattern is present (X: $\mathrm{C} 1$ and $\mathrm{C} 4$ are in contact, or + : four main cusps are in contact).

Protostylid: tubercle of various degrees of expression on the buccal surface of the lower molars. This trait is scored following ASUDAS (Turner et al., 1991).

0 . No expression of any sort.

1. A pit occurs in the buccal groove.

2. The buccal groove is curved distally.

3. A faint secondary groove extends mesially from the buccal groove.

4. The secondary groove is slightly more pronounced.

5. The secondary groove is stronger and can be easily seen.

6 . The secondary groove extends across most of the buccal surface of cusp 1 . This is considered a weak or small cusp.

7. A cusp with a free apex occurs. 
Our list of dental traits and grades are broadly based on the Arizona State University Dental Anthropology System (ASUDAS; Turner et al., 1991), but we present some modifications to better cover the variability observed in hominin species different from $H$. sapiens. The ASUDAS was developed to cover dental variability in modern populations, but it fails to cover the morphological variability observed in the genus Homo when Pleistocene fossils are included. In somecases, the ASUDAS does not cover the full range of expression that is observed for the analyzed traits (e.g.. the maximum labial convexity of incisors that is registered in the ASUDAS plaques is below the degree of labial convexity that is usually observed in Neanderthals). In other cases, the identification of the intermediate grades of some of the ASUDAS scales and plaques (e.g., variation in the lingual cusps of premolars or canine distal accessory ridge) is conf using or too subjective since hominin species present their own anatomical variations. Thus, in most of the cases we have simplified the grade-scales. Our modified version of the ASUDAS covers the dental variability of the anterior vs. posterior dentition and the maxillary vs. mandibular dentition, allowing for a complete appreciation of the whole dentition.

Along with a morphological description of the samples from the SH site and their comparison by tooth class, the frequencies of the grades of trait expression are also provided. Although both antimeres were analyzed when present, for the frequencies and the comparative analyses we followed the individual count method. including only the antimere with the highest degree of expression for each trait in the case of asymmetry (Turner et al., 1991).

\section{Comparative analyses}

The frequencies for each dental trait by group were determined. This allows for a description of each group based on 90 traits, and a simple qualitative comparison among samples.

To assess the similarity among groups, we performed a phenetic analysis based on 31 dental traits (Table 5). Since the expression of some dental features is correlated among teeth within a dental field (Turner et al., 1991), we only scored the dental trait on one specific tooth rather than all teeth in a class. For descriptive purposes, it is recommended to choose the most stable tooth of each dental field (Dahlberg, 1945), as we expect that it will present the highest expression of that trait. However, for comparative purposes, the focal tooth may be different from the most stable one, as it is recommended to choose the one that shows a more characteristic pattern of variation among the studied groups (Scott and Turner. 1997). In our study, the choice of the focal tooth was broadly based on Scott and Turner's (1997) protocol, with the following exceptions: we have scored the middle and the distal trigonid crests in $\mathbf{M}_{3}$, deflecting wrinkle in $\mathbf{M}_{2}, \mathrm{C} 6$ in $\mathbf{M}_{2}, \mathbf{C 7}$ in $\mathbf{M}_{3}$, and multiple lingual cusps in $\mathrm{P}_{3}$, instead of in the most stable tooth of the dental field, as their frequencies in those dental classes are more informative to differentiate between $H$. sapiens and H. neanderthalensis (see Bailey, 2002a,b; Martinón-Torres, 2006; Bailey et al., 2011). Finally, in order to enlarge the sample size, we have recorded the mesial marginal accessory tubercles and the transverse crest in $\mathrm{M}^{3}$ and the $\mathrm{C5}$ in $\mathrm{M}^{2}$, since these traits are easily worn out in the $\mathrm{M}^{2}$ and the $\mathrm{M}^{1}$, respectively.

The comparative phenetic analysis among populations was based on the mean measure of divergence (MMD) of C.A.B. Smith (Berry and Berry, 1967), a commonly used distance statistic in dental studies (e.g.. Irish, 1998; Bailey, 2002b; Irish and GuatelliSteinberg, 2003; but see Irish, 2010, for its limitations). We employed the MMD statistic program written by R.C. Williams. MMD analysis provides a measure of phenetic similarity based on the selected suite of dental traits, but it requires that the dental traits are dichotomized into categories of either present or absent.
Table 5

Iist of dental traits included in the MMD analysis and the breakpoint for determining absence/presence.

\begin{tabular}{|c|c|c|}
\hline Dental trait & Tooth & Presence \\
\hline Ia bial convexity & $1^{2}$ & Grades $3-5$ \\
\hline Dental tubercle & $1^{2}$ & Grades 3-6 \\
\hline Shovel shape & $\mathrm{I}^{1}$ & Grades 3-6 \\
\hline Mesial canine ridge & $\mathrm{C}$ & Grades 1-2 \\
\hline Distal accessory ridge & $C^{\prime}$ & Grades $1-2$ \\
\hline Buccal essential crest & $p^{3}$ & Grade 2 \\
\hline Lingual essential crest & $p^{d}$ & Grade 2 \\
\hline Transverse crest & $\mathrm{p}^{3}$ & Grade 2 \\
\hline Mesial accessory ridge & $\mathrm{P}^{3}$ & Grade 1 \\
\hline Distal accessory ridge & $\mathrm{p}^{3}$ & Grade 1 \\
\hline Metacone & $M^{2}$ & Grades 3-5 \\
\hline Hypocone & $M^{2}$ & Grades 3-5 \\
\hline $\mathrm{C} 5$ & $M^{2}$ & Grades $3-4$ \\
\hline Crista obliqua & $M^{2}$ & Grade 1 \\
\hline Transverse crest & $M^{3}$ & Grade 1 \\
\hline Marginal accessory tubercles & $M^{3}$ & Grade 1 \\
\hline Carabelli complex & $M^{1}$ & Grades $3-7$ \\
\hline Parastyle & $M^{3}$ & Grades 3-6 \\
\hline Premolar lingual cusp & $P_{3}$ & Grades $3-5$ \\
\hline Transverse crest & $\mathrm{P}_{4}$ & Grade 2 \\
\hline Mesial accessory ridge & $\mathrm{P}_{3}$ & Grade 1 \\
\hline Distal accessory ridge & $\mathrm{P}_{4}$ & Grade 1 \\
\hline Anterior fovea & $\mathrm{M}_{1}$ & Grade 2 \\
\hline Middle rrigonid $\alpha$ est & $M_{3}$ & Grade 2 \\
\hline Distal rrigonid crest & $M_{1}$ & Grade 2 \\
\hline Deflecting wrinkle & $M_{2}$ & Grades 1-2 \\
\hline Hypoconulid & $M_{2}$ & Grades $3-5$ \\
\hline $\mathrm{CG}$ & $M_{2}$ & Grades $2-3$ \\
\hline $\mathrm{C7}$ & $M_{3}$ & Grades $2-4$ \\
\hline Groove pattern & $\mathrm{M}_{2}$ & Grade 1 \\
\hline Protostylid & $M_{3}$ & Grades $3-7$ \\
\hline
\end{tabular}

We followed standard procedures for defining breakpoints of presence vs. absence (see Turner, 1987). However, to better differentiate among samples, some high-frequency characteristic traits were considered to be present at a higher rank (e.g., labial convexity in $\mathrm{I}^{1}$ is considered to be present at grades $\left.3-6\right)$. The dental traits included in the phenetic comparison, and the absence-presence breakpoint for each, are listed in Table 5 (for alternative breakpoints, see Irish, 1993).

Divergence between two samples was considered significant at the 0.25 level of probability when the MMD is greater than twice the standard deviation (Sjøvold, 1973). After the distance values among samples were calculated, we presented them by multidimensional scaling (PASI ${ }^{\oplus}$ software; Hammer et al., 2001), which is considered one of the most parsimonious ways to illustrate these distances (see Irish and Guatelli-Steinberg, 2003). The multidimensional scaling procedure (MDS) is a data reduction technique that attempts to find the structure in a set of distance measures among cases. The distances among individuals are illustrated in a plot, such as in a map (Kruskal and Wish, 1978) in which the proximity between samples illustrates the levels of phenetic affinity. The MDS procedure was also employed to explore Sima de los Huesos intra-population variability. Thus, we calculated the distance between each pair of individuals from the $H$. neanderthalensis, $H$. heidelbergensis, and Sima de los Huesos samples for each dental class. We employed the Gower coefficient as it can combine ordinal and nominal variables. Through the inspection of the MDS plot, we can compare the degree of "neanderthalization" of each individual from the $\boldsymbol{H}$. heidelbergensis and Sima de los Huesos samples and examine whether or not all of them fall into the Neanderthal distribution. Those individuals with more than one missing value were not included in the MDS analysis. In some cases, the number of variables included in the MDS analysis was reduced in order to maximize the number of 
individuals per hominin group, particularly in the case of H. heidelbergensis.

\section{Measurement definitions}

In addition to dental morphological observations, the mesiodistal (MD) and buccolingual (BL) dimensions of the Sima de los Huesos teeth were measured by JMBC to the nearest $0.1 \mathrm{~mm}$, following the methods of Flechier, Lefêvre, and Verdéne (Lefêvre, 1973; see Martinón-Torres et al., 2008, for a more detailed explanation) (SOM Table 1). This method can be applied identically to both isolated and in situ teeth. Additionally, this method has been shown to have a low intraobserver error because it employs a reference plane that can be easily identified by different observers. Measurements were taken between the contact points in normal occlusion or where these points would have been (in the case of malpositioned teeth). The diameters of those teeth with occlusal wear $\geq 6$ (Molnar, 1971) were not included in the calculation of the average tooth class diameters (Table 6).

\section{Results}

\section{Description of the SH teeth}

Upper central incisors $\left(\mathrm{I}^{1}\right)$ All of the $\mathrm{I}^{1} \mathrm{~s}$ in the sample are generally well-preserved (Figs. 1 and 2; Table 7). The enamel is chipped at the incisal edge of AT-8 and in the distobuccal area of AT-146. The root apices of AT-27, AT-2395, and AT-42 are missing.

From the labial and lingual aspects, the crowns are trapezoidal with curved sides that become parallel toward the incisal edge. The mesial incisal angle is slightly straighter than the distal angle. All $\mathrm{I}^{1} \mathrm{~s}$ show some degree of shovel shape, but only $60 \%$ present moderately developed marginal ridges (grade 4 ). In $30 \%$ of individuals, the expression is mild (grade 3 ), and the highest grades of expression for this trait (grades 5 and 6 ) are absent in the SH sample. The shovel shape is generally symmetric, with a slightly thicker mesial marginal ridge in some isolated cases (e.g., AT-3193, AT-3194, AT278). In general, there is no lingual fossa associated with the shovel shape, due to the constant expression of a large, round basal eminence that occupies from one- to two-thirds of the lingual surface. There is a moderate tuberculum dentale (grade 3 ) in the shape of enamel folds and depressions in only $25 \%$ of the specimens (e.g., AT-954, AT-280). From the occlusal aspect, all of the $\mathrm{I}^{1} \mathrm{~s}$ show the highest degrees of mesiodistal convexity (grade $\geq 4$ ). The massive aspect of the tooth can be noted in lateral view.

Table 6

Average MD and BL diameters for each SH tooth class. Those teeth with occlusal wear $>6$ were not included. Measures are in milimeters $(\mathrm{mm})$.

\begin{tabular}{|c|c|c|c|c|c|c|}
\hline \multirow[t]{2}{*}{ Tooth class } & \multicolumn{3}{|c|}{ MD } & \multicolumn{3}{|c|}{$\mathrm{BL}$} \\
\hline & Mean & SD & $\mathrm{N}$ & Mean & SD & $\mathrm{N}$ \\
\hline $\mathrm{I}^{1}$ & 9.64 & 0.57 & 24 & 7.75 & 0.34 & 24 \\
\hline $\mathrm{I}^{2}$ & 7.78 & 0.37 & 24 & 7.76 & 0.28 & 24 \\
\hline$C^{\prime}$ & 8.75 & 0.38 & 23 & 9.88 & 0.52 & 24 \\
\hline $\mathrm{P}^{3}$ & 7.81 & 0.51 & 24 & 10.38 & 0.66 & 25 \\
\hline $\mathrm{P}^{4}$ & 7.55 & 0.53 & 24 & 10.27 & 0.65 & 26 \\
\hline $\mathrm{M}^{1}$ & 11.05 & 0.68 & 26 & 11.5 & 0.62 & 26 \\
\hline$M^{2}$ & 9.94 & 0.94 & 28 & 12.18 & 0.73 & 28 \\
\hline $\mathrm{M}^{3}$ & 8.62 & 0.6 & 24 & 11.46 & 0.92 & 25 \\
\hline $\mathrm{I}_{1}$ & 5.59 & 0.18 & 26 & 6.56 & 0.31 & 26 \\
\hline $\mathrm{I}_{2}$ & 6.61 & 0.27 & 30 & 7.3 & 0.34 & 30 \\
\hline$C$ & 7.63 & 0.43 & 29 & 8.66 & 0.66 & 29 \\
\hline$P_{3}$ & 7.91 & 0.39 & 33 & 8.98 & 0.54 & 32 \\
\hline $\mathrm{P}_{4}$ & 7.17 & 0.44 & 35 & 8.57 & 0.59 & 35 \\
\hline $\mathrm{M}_{1}$ & 11.25 & 0.49 & 39 & 10.45 & 0.46 & 38 \\
\hline $\mathrm{M}_{2}$ & 11.06 & 0.56 & 39 & 10.23 & 0.67 & 39 \\
\hline$M_{3}$ & 11.28 & 0.81 & 38 & 9.76 & 0.72 & 38 \\
\hline
\end{tabular}

The root is single, long, robust, and subtriangular in transverse section. The four surfaces (labial, lingual, mesial, and distal) are well-defined. In general, there is a shallow longitudinal depression along the distal surface. From the labial and lingual surfaces, the apical third of the root diverges slightly distally.

Upper lateral incisors $\left(\mathrm{I}^{2}\right)$ All of the $\mathrm{SH} \mathrm{I}^{2} \mathrm{~s}$ are well-preserved (Figs. 3 and 4; Table 8). There is superficial enamel loss on the labial surface of AT-53 and a slight loss of cement in the root of AT-29. There is a fracture in the apical third of the roots of AT1124 and AT-283.

From both the labial and lingual aspects, the $\mathrm{I}^{2}$ crowns show a sub-rectangular contour with nearly parallel marginal ridges. All of the specimens present a conspicuous and characteristic shovel shape, where the marginal ridges are extremely thickened and invade the lingual fossa, circumscribing in almost all of the specimens a narrow longitudinal groove. This groove is usually centered, but it can also be somewhat deviated mesially (e.g., AT-2769, AT2279, AT-1754). All of the $\mathrm{I}^{2} \mathrm{~s}$ show a moderate basal eminence, though this is less pronounced than in the $I^{1}$ s. A moderate- to strongly-developed tuberculum dentale (grade $\geq 3$ ) is evident in $58 \%$ of specimens. This tubercle can adopt the shape of a rectangular platform and cover the apical end of the longitudinal lingual groove, delimiting the expression of a deep foramen caecum (e.g., AT-820). The tuberculum dentale can be continuous with the marginal ridges (e.g., AT-7, AT-53) or separated by accessory mesial (e.g., AT-1444), distal (e.g., AT-1962), or both mesial and distal grooves (e.g., AT-820, AT-962).

From the occlusal aspect, all of the $\mathrm{I}^{2} \mathrm{~s}$ present the maximum degree of labial convexity (grade 5). This strong mesiodistal convexity in combination with the deep longitudinal lingual groove provides a characteristic " $V$ " occlusal section, also known as "triangular shovel shape" (Martinón-Torres et al., 2007a,b). Individual XVIII presents a more classical shovel shape with a more spacious lingual fossa, but the presence of a lingual longitudinal groove of varied depth is consistent throughout the sample.

From the lateral view, a strong lingual inclination of the labial surface can be ascertained from the basal portion toward the incisal edge. The wear plane of the incisal edge is lingually inclined, suggesting an edge-to-edge occlusion (Bermúdez de Castro, 1987). The cemento-enamel junction curvature is less pronounced on the distal side. From this view, the massive aspect of the tooth is obvious.

The root is robust, subtriangular in transverse section, and mesiodistally compressed. The labial face is broader than the lingual face, and its distal third is strongly inclined lingually. Broad and shallow longitudinal grooves run across the mesial and distal faces of the root.

Upper canines $\left(\mathrm{C}^{\prime}\right)$ The $\mathrm{C}^{\prime}$ 's of the $\mathrm{SH}$ sample are excellently preserved (Fig. 5 and Table 9). There is a small amount of superficial enamel loss on the labial surf ace of AT-995, on the mesial surf ace of AT-818, on the lower and mesial quadrant of AT-1475, and on the labial aspect of AT-825. AT-3192 shows an enamel fracture and superficial cement loss. The distal two-thirds of the AT-1942 root are missing.

When the tooth is unworn or barely worn, the crown has a pentagonal shape in labial view, with its base at the cementoenamel line and the vertex toward the occlusal rim. The mesial arm of the upper triangle is slightly shorter than the distal one, and the apex tends to be mesially displaced. This vertex can be totally worn so that the crown adopts a trapezoidal shape with a rectilinear occlusal rim.

From the lingual view, all of the C's exhibit moderate to strong shovel shapes (e.g., AT-2151, AT-1942) (grade $\geq 3$ ). The thickened mesial and distal marginal ridges are round in cross-section and well-developed, especially in the region of the basal eminence. 


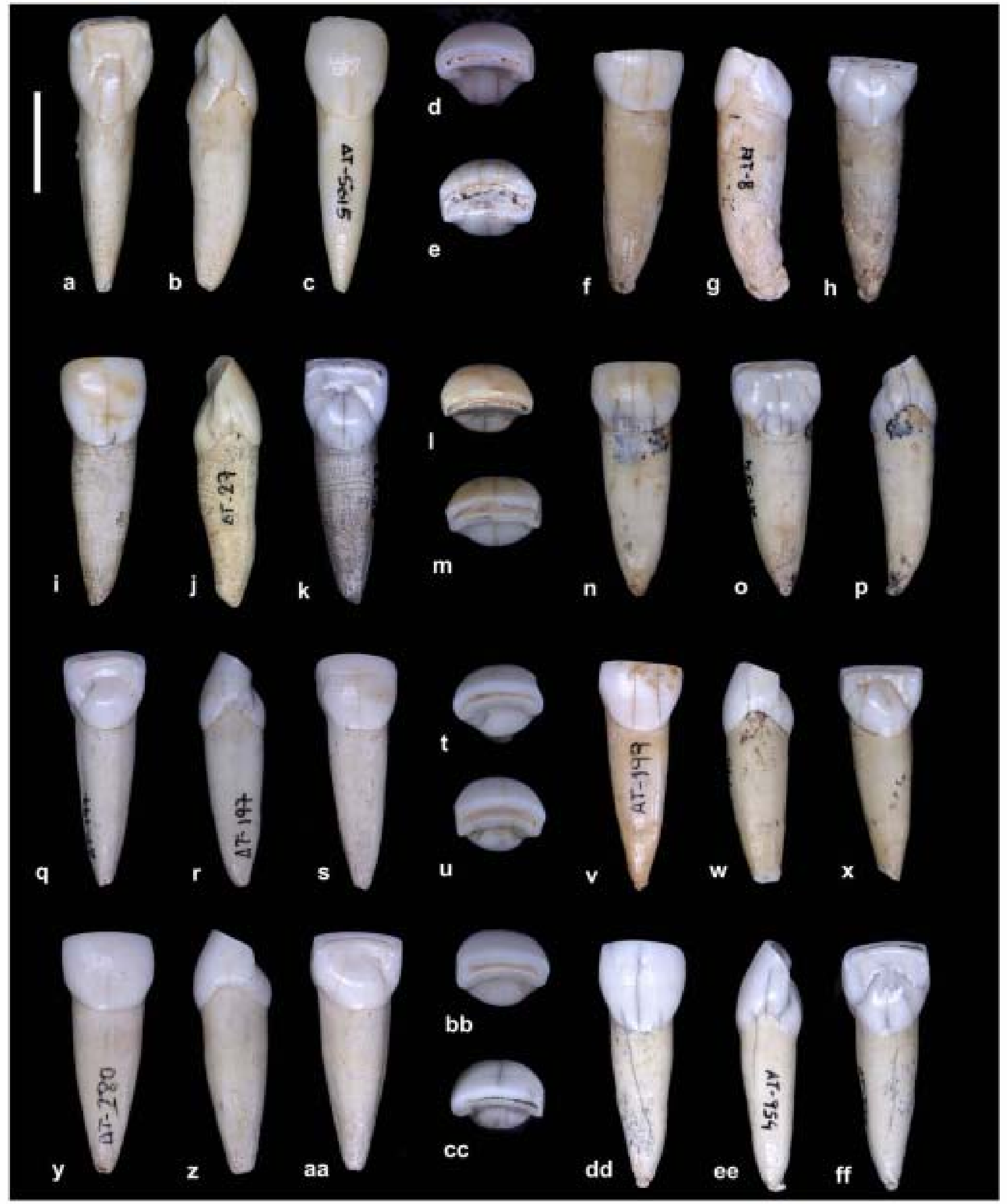

Figure 1. The SH I ${ }^{1} \mathrm{~s}$. Lingual (a), mesial (b), labial (c), and occlusal (d) aspect of AT-5615 (R); occlusal (e), labial (f), mesial (g), and lingual (h) aspect of AT-8 (L); labial (i), distal (j), lingual (k), and occlusal (I) aspect of AT-27 (L); occlusal (m), lingual ( $\mathrm{n})$, labial (o), and mesial (p) aspect of AT-54 (R); lingual ( $(\mathrm{R})$; occlusal (u), labial ( $\mathrm{v}$ ), mesial (w), and lingual (x) aspect of AT-199 (L); buccal (y), mesial (z), lingual (aa), and occlusal (bb) aspect of AT-280 (L); occlusal (cc), labial (dd), distal (ee), and lingual (ff) aspect of AT-954 (R). L: left, R: right. White bar $=1 \mathrm{~cm}$. 


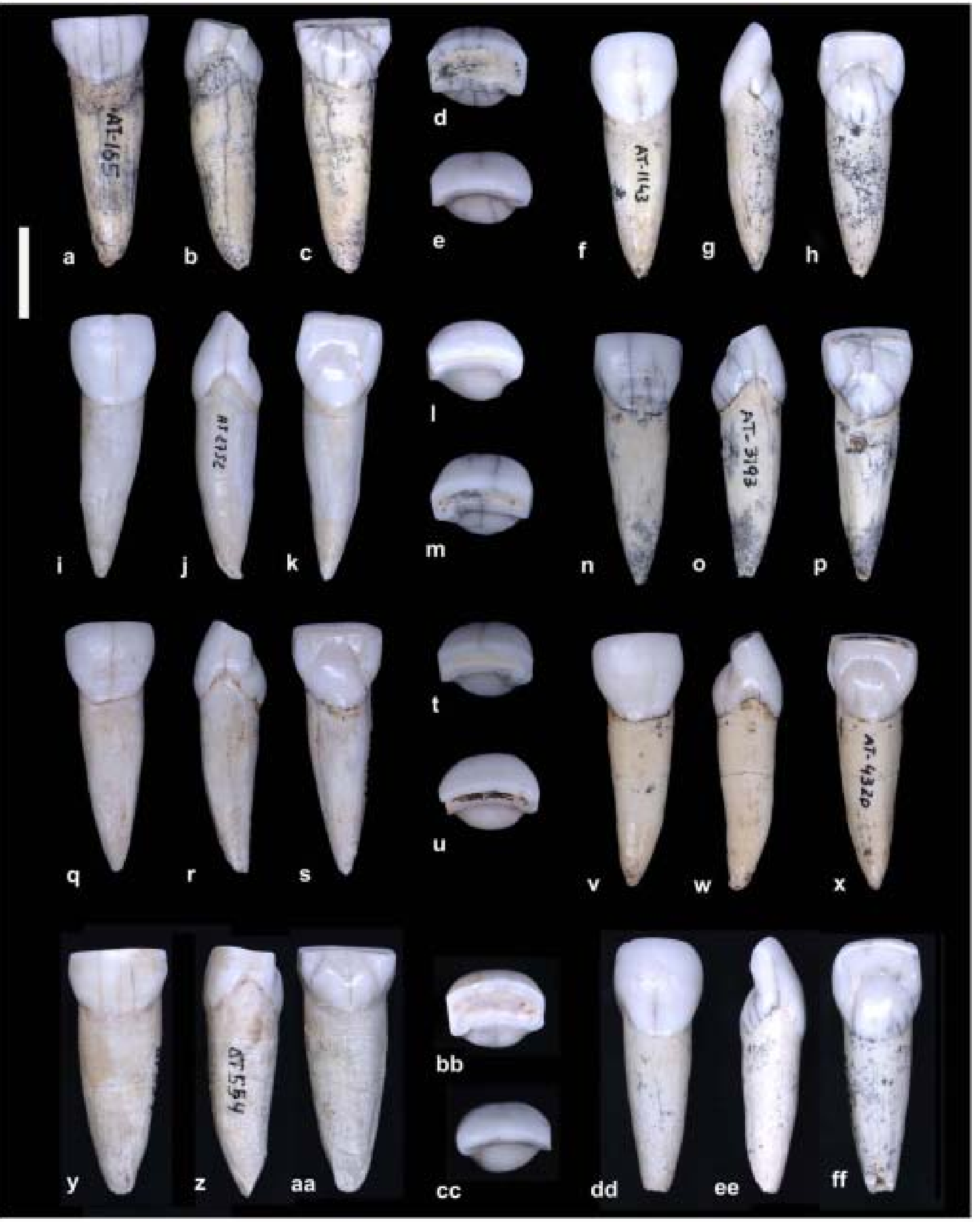

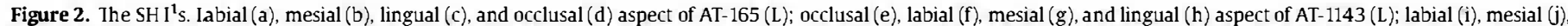

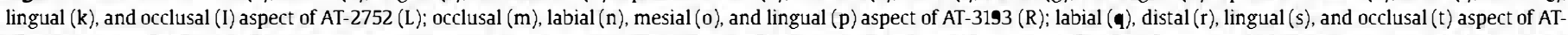

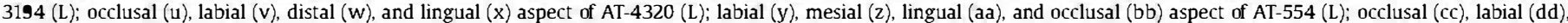
mesial (ee), and lingual (ff) aspect of AT-2395 (R). L: left, R: right. White bar $=1 \mathrm{~cm}$. 
Table 7

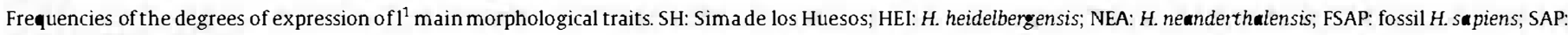
contemporary $H$. sapiens.

\begin{tabular}{|c|c|c|c|c|c|c|}
\hline $\mathrm{I}^{1}$ & Grade & SH & HEI & NEA & FSAP & SAP \\
\hline \multirow[t]{7}{*}{ Labial convexity } & 0 & 0 & 0 & 0 & $5(29.4 \%)$ & $33(36.3 \%)$ \\
\hline & 1 & 0 & 0 & 0 & $1(5.9 \%)$ & $30(33 \%)$ \\
\hline & 2 & 0 & 0 & 0 & $6(35.3 \%)$ & $22(24.2 \%)$ \\
\hline & 3 & 0 & $1(100 \%)$ & $2(9.5 \%)$ & $3(17.6 \%)$ & $6(6.6 \%)$ \\
\hline & 4 & $10(50 \%)$ & 0 & $10(47.6 \%)$ & $1(5.9 \%)$ & 0 \\
\hline & 5 & $10(50 \%)$ & 0 & $9(42.9 \%)$ & $1(5.9 \%)$ & 0 \\
\hline & Total & 20 & 1 & 21 & 17 & 91 \\
\hline \multirow[t]{6}{*}{ Tuberculum dentale } & 0 & 0 & 0 & 0 & $2(12.5 \%)$ & $45(50 \%)$ \\
\hline & 1 & $10(50 \%)$ & $1(50 \%)$ & $2(9.5 \%)$ & $3(18.8 \%)$ & $21(23.3 \%)$ \\
\hline & 2 & $5(25 \%)$ & 0 & $2(9.5 \%)$ & $7(43.8 \%)$ & $13(14.4 \%)$ \\
\hline & 3 & $5(25 \%)$ & 0 & $6(28.6 \%)$ & $3(18.8 \%)$ & $8(8.9 \%)$ \\
\hline & 4 & 0 & $1(50 \%)$ & $11(52.4 \%)$ & $1(6.3 \%)$ & $3(3.3 \%)$ \\
\hline & Total & 20 & 2 & 21 & 16 & 90 \\
\hline \multirow[t]{8}{*}{ Shovel shape } & 0 & 0 & 0 & 0 & $1(5.9 \%)$ & $16(17.6 \%)$ \\
\hline & 1 & $1(5 \%)$ & 0 & 0 & $1(5.9 \%)$ & $28(30.8 \%)$ \\
\hline & 2 & $1(5 \%)$ & $1(25 \%)$ & $2(9.5 \%)$ & $4(23.5 \%)$ & $27(29.7 \%)$ \\
\hline & 3 & $6(30 \%)$ & $2(50 \%)$ & $5(23.8 \%)$ & $7(41.2 \%)$ & $15(16.5 \%)$ \\
\hline & 4 & $12(60 \%)$ & $1(25 \%)$ & $4(19 \%)$ & $4(23.5 \%)$ & $3(3.3 \%)$ \\
\hline & 5 & 0 & 0 & $7(33.3 \%)$ & 0 & $1(1.1 \%)$ \\
\hline & 6 & 0 & 0 & $3(14.3 \%)$ & 0 & $1(1.1 \%)$ \\
\hline & Total & 20 & 4 & 21 & 17 & 91 \\
\hline
\end{tabular}

When the shovel shape is pronounced, the marginal ridges are differentiated from the canine essential ridge by clear marginal grooves. In some cases, a lingual longitudinal groove, similar to the groove expressed in the $\mathrm{I}^{2} \mathrm{~s}$, can be seen. This groove is different from the interruption groove, which transects the basal eminence and the root (Scott and Turner, 1997). With only one exception (AT1758), the mesial marginal ridge is always thicker than the distal one, and in $79 \%$ of the sample, it is fused with the tuberculum dentale (Morris' type). The distal marginal ridge tends to be shorter than the mesial one, and, in some cases, it adopts the shape of a tubercle, ending in the apical third of the crown. The tuberculum dentale presents great variability, ranging from medium in size or grade $3(28.6 \%$ ) to more conspicuous forms (grade $\geq 4$ ) in $42.8 \%$ of the sample. In $9.5 \%$ of cases, only a swelling of the basal eminence can be ascertained. In many specimens, the mesial marginal ridge incorporates the tuberculum dentale, and, therefore, it cannot be clearly identified. In 33\% of the sample, the tubercle is clearly differentiated by marginal grooves and presents a free apex (e.g., AT-2151, AT-1757, AT-958).

In half of the cases we examined, there is a distal accessory ridge located between the essential ridge and the distal marginal ridge. In $14.3 \%$, its expression is mild (grade 1), whereas in $35.7 \%$ of the sample this ridge is pronounced (grade 2 ).

From the lateral view, some specimens show a moderate basal swelling of the labial surface (e.g., AT-6, AT-144), but a cingulum is not expressed. The labial surface is strongly inclined lingually from the basal eminence area to the incisal plane.

The root is single and robust. It exhibits an oval section that is mesiodistally compressed, with the buccal face being broader than the lingual. The apical portion is lingually inclined. A strong, wide longitudinal groove runs along the medial and distal thirds of the root on both the mesial and distal faces.

Upper third premolars $\left(\mathrm{P}^{3}\right)$ The $\mathrm{P}^{3} \mathrm{~s}$ of the SH sample are generally well-preserved (Fig. 6 and Table 10). AT-4332 shows a generalized mottled enamel. The apex of the lingual root of AT-823 and the apical two-thirds of the roots of AT-2036, AT-24, AT-1944, and AT2399 are fractured.

The occlusal outline of this tooth type is a mesiodistally compressed oval, with the buccal half being broader than the lingual one. All of the $\mathrm{P}^{3} \mathrm{~s}$ display the two main cusps, with the buccal cusp being invariably larger than the lingual one. In $80 \%$ of specimens, the apex of the lingual cusp is mesially deviated with regard to the apex of the buccal one. In almost half of the $\mathrm{P}^{3} \mathrm{~s}$ in the sample, the essential ridge of the buccal cusp is flat (grade 0), in $29.4 \%$ it is single, and in $23.5 \%$ of cases it is bifurcated (grade 2 ). Only four individuals show a distal accessory ridge, and no individual presents a mesial one. On the lingual cusp, almost 90\% of individuals have a single essential ridge (grade 1 ); in only two cases are the lingual crests bif urcated (AT-813, AT-405). In individual XX, there is a continuous transverse crest that is mesially displaced, connecting the main cusps (grade 2). With this exception, the $\mathrm{SH}$ $\mathrm{P}^{3}$ s show an uninterrupted sagittal fissure that is usually short and rectilinear. This fissure ends in anterior and posterior foveae that are generally shallow, pit-like, or linear, but which in all cases are simple. In the majority of the specimens, the sagittal fissure bifurcates at the mesial and distal ends, delimitating minor accessory tubercles. Despite their relative high frequencies (Table 10), their expression is weak, and, therefore, the occlusal aspect of the teeth is simple and does not show enamel wrinkles or secondary fissures.

From the lateral view, the upper half of the buccal face shows a moderate to strong bulge, but there are no signs of a buccal cingulum. This swelling is particularly marked at the mesial zone, where a tuberculum molare is clearly identifiable.

More than half of the $\mathrm{P}^{3} \mathrm{~S}$ are double-rooted (64.3\%). The lingual and buccal roots coalesce in their cervical third, and are wellseparated in the apical two-thirds but are joined by a mesial sheet of dentine and/or cementum. The buccal root tends to be shorter and more rectilinear, while the lingual root is longer and has a lingual convexity in its medium third. Even when $\mathrm{P}^{3}$ is singlerooted, a broad and deep groove can be found separating the mesial and the distal surfaces.

Upper fourth premolars $\left(\mathrm{P}^{4}\right)$ The SH $\mathrm{P}^{4}$ s are well-preserved (Fig. 7 and Table 11). Only the apices of both roots of AT-193 and AT-559 and the apices of the buccal roots of AT-23 and AT-68 are missing.

The occlusal outline is a rounded rectangle that is more symmetrical than that of the $\mathrm{P}^{3} \mathrm{~s}$. The $\mathrm{BL}$ axis is longer than the MD axis. All of the $\mathrm{P}^{4} \mathrm{~s}$ display the two main cusps, with the buccal cusp generally being larger than the lingual one, although the size difference between both cusps is less pronounced than in the $\mathrm{P}^{3} \mathrm{~s}$. In $73.7 \%$ of cases, the tip of the lingual cusp is mesially deviated with regard to the buccal cusp, and, in the remaining specimens, both tips are centered. In approximately two-thirds of the sample, the 

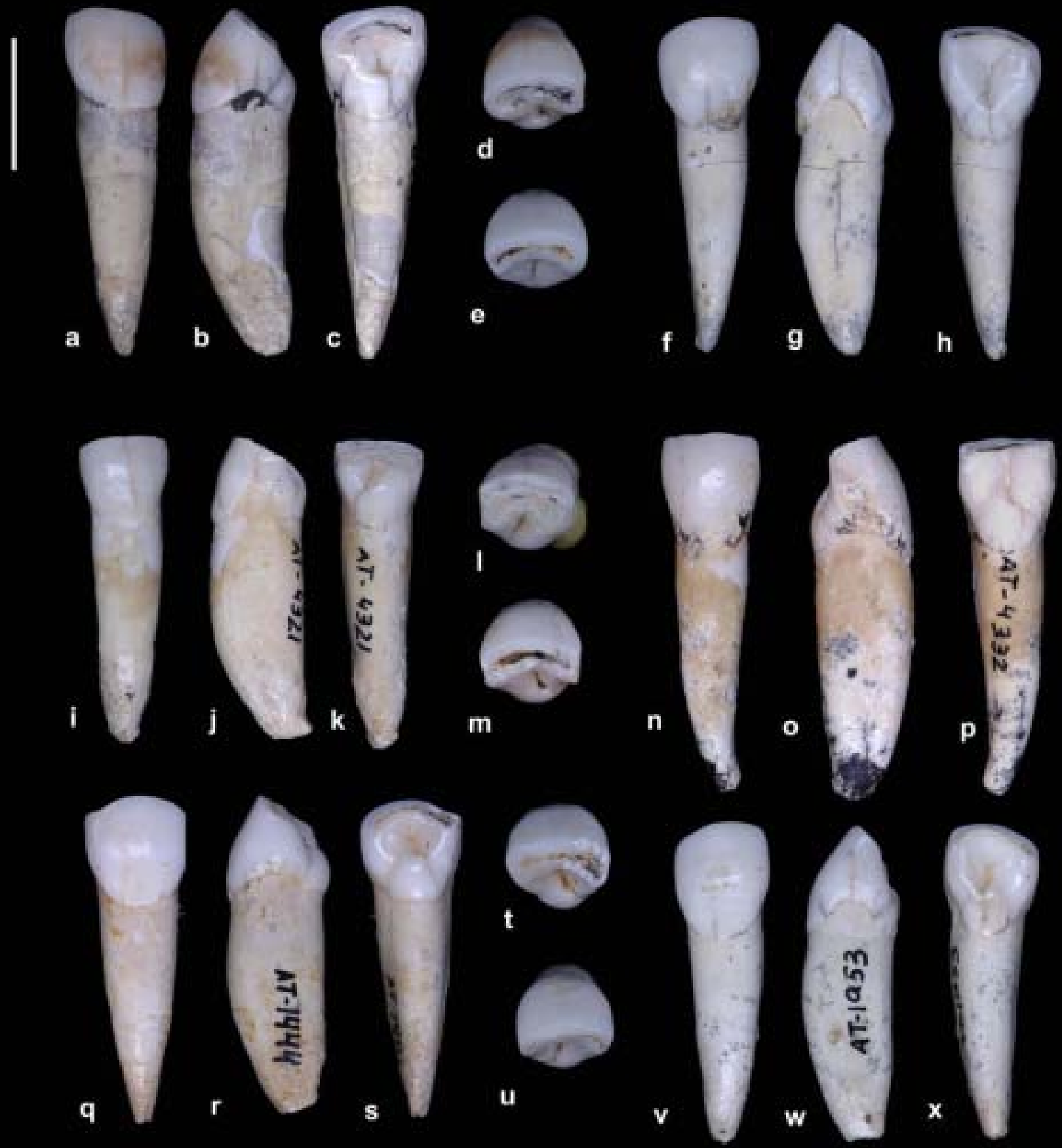

Figure 3. The SH $\mathrm{I}^{2}$ s. Labial (a), mesial (b), lingual (c), and occlusal (d) aspect of AT-29 (R); occlusal (e), labial (f), mesial (g), and lingual (h) aspect of AT-961 (L); labial (i), mesial (j), lingual $(\mathrm{k})$, and occlusal $(\mathrm{I})$ aspect of AT-4321 (L); occlusal $(\mathrm{m})$, labial $(\mathrm{n})$, mesial $(\mathrm{o})$, and lingual $(\mathrm{p})$ aspect of AT-4332 (R); labial ( $\mathbf{q})$, distal $(\mathrm{r})$, lingual (s), and occlusal (t) aspect of AT$1444(\mathrm{R})$; occlusal $(\mathrm{u})$, labial $(\mathrm{v})$, mesial $(\mathrm{W})$, and lingual $(\mathrm{x})$ aspect of AT-1953 (L). L: left, R. right. White bar $=1 \mathrm{~cm}$.

essential ridge of the buccal cusp is single and not very pronounced (grade 1), while in one-third it is bif urcated (grade 2 ), particularly where it contacts the sagittal fissure (e.g., AT-2070). A mesial accessory ridge in the buccal cusp is present in $46 \%$ of the sample, and $46 \%$ of the specimens also have a distal ridge, though there is no significant correlation of the expression of both in the same specimen ( $p$-value $=2.84$ ). For the lingual cusp, half of the sample presents a single essential ridge (grade 1 ) that is generally more conspicuous than the buccal ridge. The other half of the sample presents a bifurcation (e.g., AT-409, AT-559).
A continuous sagittal fissure separates the main cusps in almost $80 \%$ of the specimens. This fissure runs from the anterior to the posterior fovea and is generally deeper but still shallow, pit-like, or linear. The sagittal fissure bifurcates at its mesial and/or distal ends, crossing the marginal ridges in some cases, and sometimes delimitating weak accessory tubercles. The number and location of these accessory tubercles is highly variable. Approximately half of the individuals in the sample have both mesial and distal tubercles. At least one distal accessory tubercle is present in $94.3 \%$ of the sample vs. $70.7 \%$ that have at least one mesial tubercle. These 


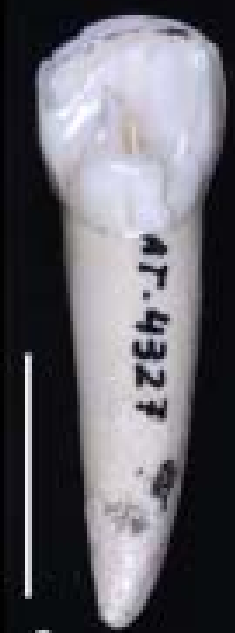

a

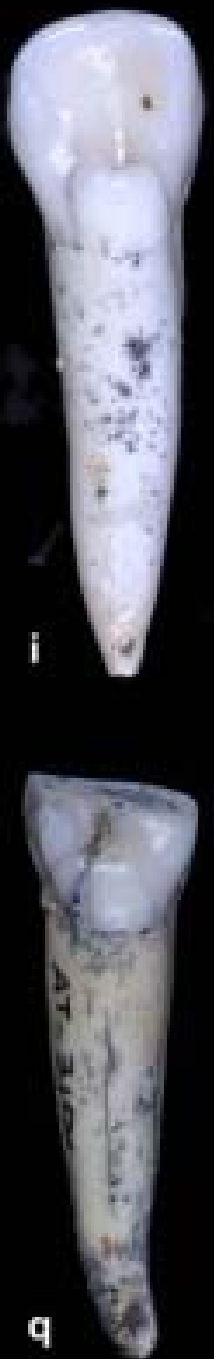

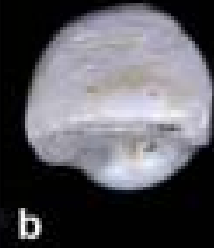

C

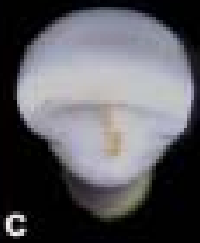

d
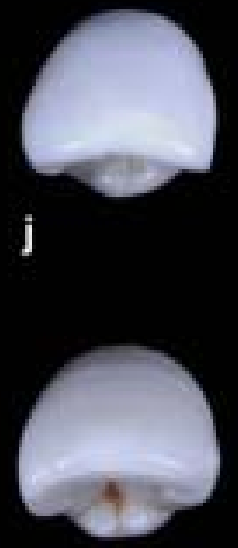

k

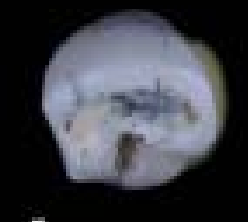

r

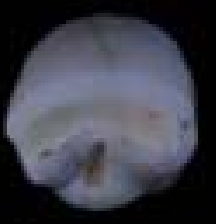

s
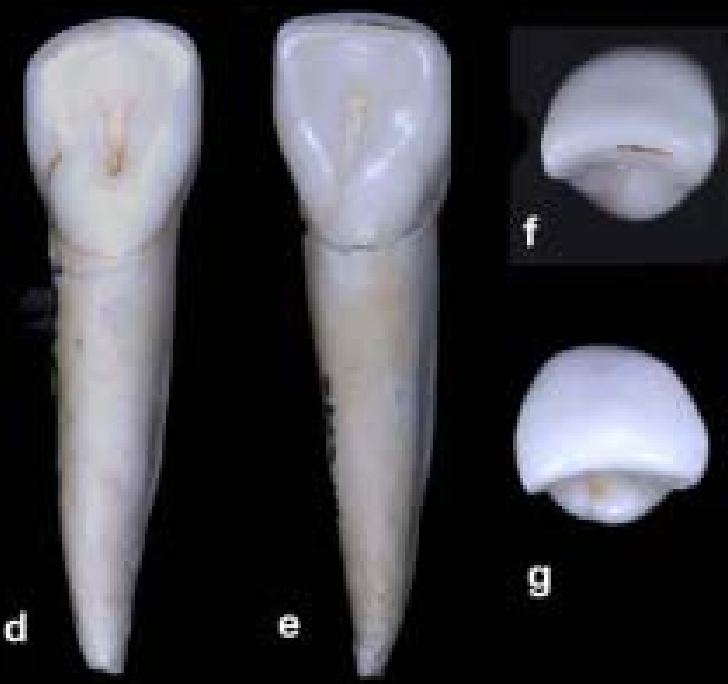

g

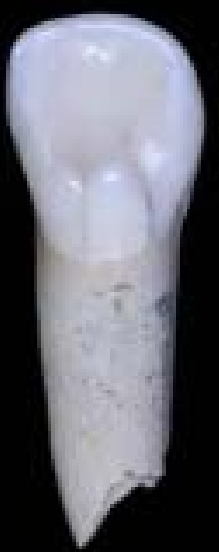

h
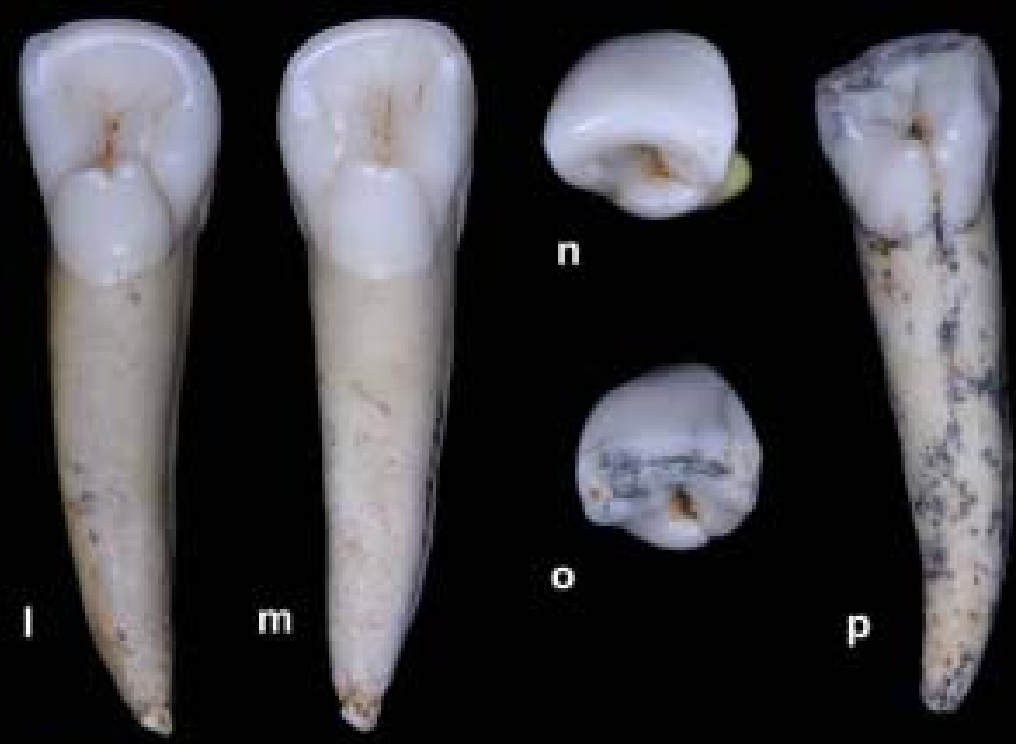

o
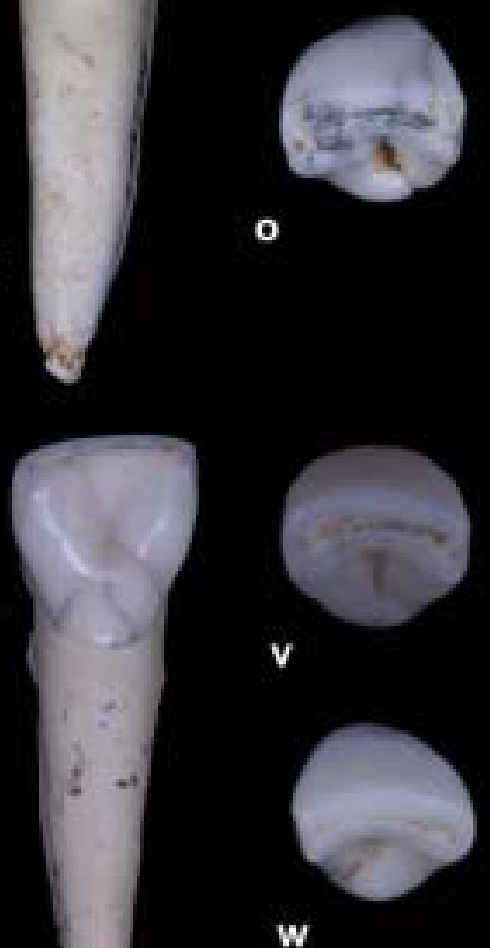

u

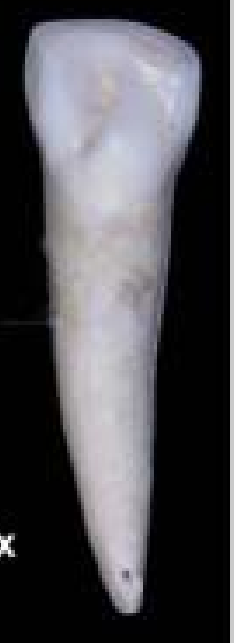

Figure 4. The SH $I^{2}$ s. Lingual (a) and occlusal (b) aspect of AT-4327 (R); occlusal (c) and lingual (d) aspect of AT-2769 (R); lingual (e) and occlusal (f) aspect of AT-2272 (L); occlusal $(\mathrm{g})$ and lingual $(\mathrm{h})$ aspect of AT- 1124 (L); lingual (i) and occlusal (j) aspect of AT-2280 (R); occlusal (k) and lingual aspect (I) of AT-820 (L); lingual (m) and occlusal (n) aspect of AT$962(\mathrm{R})$; occlusal $(\mathrm{o})$ and lingual $(\mathrm{p})$ aspect of AT-3195 (R); lingual $(\mathbf{q})$ and occlusal $(\mathrm{r})$ aspect of AT-3196 (L); occlusal (s) and lingual (t) aspect of AT-7 (L); lingual (u) and occlusal $(\mathrm{v})$ aspect of AT-5608 (R); occlusal (W) and lingual ( $\times$ ) aspect of AT-1754 (L). L: left, R: right. White bar $=1 \mathrm{~cm}$. 


\begin{tabular}{|c|c|c|c|c|c|c|}
\hline $\mathrm{I}^{2}$ & Grade & SH & HEI & NEA & FSAP & SAP \\
\hline \multirow[t]{7}{*}{ Labial convexity } & 0 & 0 & 0 & 0 & 0 & $39(31.5 \%)$ \\
\hline & 1 & 0 & 0 & 0 & $2(28.6 \%)$ & $35(28.2 \%)$ \\
\hline & 2 & 0 & 0 & $1(3.2 \%)$ & 0 & $38(30.6 \%)$ \\
\hline & 3 & 0 & 0 & $1(3.2 \%)$ & $2(28.6 \%)$ & $12(9.7 \%)$ \\
\hline & 4 & 0 & $2(100 \%)$ & $9(29 \%)$ & $2(28.6 \%)$ & 0 \\
\hline & 5 & $17(100 \%)$ & 0 & $20(646 \%)$ & $1(143 \%)$ & 0 \\
\hline & Total & 17 & 2 & 31 & 7 & 124 \\
\hline \multirow[t]{8}{*}{ Tuberculum dentale } & 0 & $2(10.5 \%)$ & $2(100 \%)$ & 0 & 0 & $62(50 \%)$ \\
\hline & 1 & $2(10.5 \%)$ & 0 & $1(3.3 \%)$ & $4(57.1 \%)$ & $25(20.2 \%)$ \\
\hline & 2 & $4(21.1 \%)$ & 0 & 0 & $1(143 \%)$ & $21(16.9 \%)$ \\
\hline & 3 & $5(26.3 \%)$ & 0 & $2(6.7 \%)$ & $2(28.6 \%)$ & $10(8.1 \%)$ \\
\hline & 4 & $1(5.3 \%)$ & 0 & $6(20 \%)$ & 0 & $4(3.2 \%)$ \\
\hline & 5 & $4(21.1 \%)$ & 0 & $12(40 \%)$ & 0 & $2(1.6 \%)$ \\
\hline & 6 & $1(5.3 \%)$ & 0 & $9(30 \%)$ & 0 & 0 \\
\hline & Total & 19 & 2 & 30 & 7 & 124 \\
\hline \multirow[t]{8}{*}{ Shovel shape } & 0 & 0 & 0 & 0 & 0 & $24(19 \%)$ \\
\hline & 1 & 0 & 0 & 0 & 0 & $22(17.5 \%)$ \\
\hline & 2 & 0 & 0 & $3(9.7 \%)$ & $2(28.6 \%)$ & $21(16.7 \%)$ \\
\hline & 3 & 0 & $1(50 \%)$ & $1(3.2 \%)$ & $3(42.9 \%)$ & $20(15.9 \%)$ \\
\hline & 4 & $5(25 \%)$ & 0 & $2(6.5 \%)$ & $1(14.3 \%)$ & $29(23 \%)$ \\
\hline & 5 & $9(45 \%)$ & $1(50 \%)$ & $13(41.9 \%)$ & $1(143 \%)$ & $10(7.9 \%)$ \\
\hline & 6 & $6(30 \%)$ & 0 & $12(38.7 \%)$ & 0 & 0 \\
\hline & Total & 20 & 2 & 31 & 7 & 126 \\
\hline
\end{tabular}

tubercles can be expressed in a variable quantity in only one marginal ridge (35.4\%), or it can be present in both marginal ridges at the same time $(64.8 \%)$. Despite their variable number and combinations, the tubercles are not strongly defined and the occlusal aspect of these teeth, in general, is not complex.

From the lateral aspect, the upper half of the buccal face is moderately swollen, but no traces of cingulum or of tuberculum molare can be found. The majority of the $\mathrm{P}^{4} \mathrm{~s}$ are double-rooted (85.7\%) vs. $64.3 \%$ of the $\mathrm{P}^{3}$ s. Even in the two individuals with single-rooted $\mathrm{P}^{4} \mathrm{~S}$ (individual XVI and AT-5510), a clear longitudinal groove runs along the distal face, delimiting buccal and lingual radicals. Bifurcation is closer to the cemento-enamel junction than it is in the $\mathrm{P}^{3} \mathrm{~s}$. The bifurcation can start in the first or second third of the root, but in all cases there is a lamina of dentine and/or cementum along the mesial face joining the buccal and lingual roots. This lamina is similar to that found in the $\mathrm{P}^{3}$ s but thicker (e.g., AT-23). The buccal root is more rectilinear and generally longer than the lingual one, which is buccally flexed in its most apical third.

Upper first molar $\left(\mathrm{M}^{1}\right)$ The preservation of the $\mathrm{SH} \mathrm{M}^{1} \mathrm{~s}$ is excellent (Figs. 8 and 9; Table 12), with only some radicular fractures: the distobuccal root in AT-3177, AT-2071, and AT-138 (which, together with AT-2076, also lacks the lingual root), the apical third of the mesiobuccal root of AT-20, the buccal roots in AT-196, and the apices of the distobuccal and mesiobuccal roots of AT-139 and AT26 , respectively.

The occlusal outline of all the $\mathrm{M}^{1} \mathrm{~s}$ is a rounded rhomboid with a relatively distal displacement of the lingual cusps and a rounded protrusion of the distobuccal corner. All the $\mathrm{M}^{1} \mathrm{~s}$ exhibit the four main cusps. With only one exception (individual XIX), the rest of the sample presents a large or very large metacone (grade $\geq 4$ ). All of the SH specimens present a characteristic bulging and rounded hypocone, and its remarkable development is responsible for the distobuccal protrusion in the occlusal outline. Approximately half of the sample presents a C5 in the distal margin (grade $\geq 2$ ), which is triangular in shape and delimited by a V-shaped groove that stems from the posterior fovea of the tooth. The size is variable, but it tends to be relatively small in all cases. In $25 \%$ of the sample, the C5 is clearly absent, and in $18.8 \%$ of cases the distal groove is worn away (grade 1), possibly representing the incipient development of a C5. The crista obliqua connecting the protocone and the metacone is conspicuous and invariably present in all of the specimens. The transverse crests connecting the mesial cusps as well as the mesial accessory tubercles are easily affected by wear, and, therefore, we should be cautious about assuming their real frequency. A transverse crest has been recorded in approximately half of the population (58.3\%). When this crest is strongly developed, it tends to adopt a triangular shape (e.g., AT-2071, AT-587). Half of the specimens express mesial accessory tubercles, but they are not welldefined or conspicuous.

On the lingual surface of the protocone, a slight and punctual depression can be found in $56.3 \%$ of the individuals, corresponding to the lower degree of expression (grade 1), or the so-called "negative form" of the Carabelli's complex. A similar punctual depression is found on the buccal surf ace of the paracone in $29.4 \%$ of cases, corresponding to the lower degree of expression of a parastyle (grade 1) (Irish, 1993).

The SH $\mathrm{M}^{1}$ s tend to exhibit three roots: one mesiobuccal, one distobuccal, and one lingual. The distal bifurcation point is wider than the mesial, and the buccal roots diverge further from the cemento-enamel junction than do the lingual ones. The lingual root is the largest of the three and tends to be curved toward the buccal root, while the distobuccal root is shorter and is slightly curved toward the distal root, though its apex is mesially inclined. Roots may coalesce and only diverge in the apical-most third (AT-3178), joined by a buccal lamina of dentine and/or cementum. More than half of the specimens show hypotaurodontism (61.5\%) with a slightly enlarged pulp cavity and a bifurcation beyond the first third of the root length. In AT-20, we observe a case of mesotaurodontism, where the pulp cavity occupies approximately twothirds of the total length of the root. Only individual VII presents hypertaurodontism, with a prismatic root that does not show any bifurcation.

Upper second molar $\left(\mathrm{M}^{2}\right)$ All of the $\mathrm{SH} \mathrm{M}^{2} \mathrm{~s}$ are in a state of excellent preservation (Figs. 10 and 11; Table 13). Only the distolabial portion of the crown of AT-827 and the distal twothirds of the buccal roots of AT-407 are missing.

The occlusal contour of the $\mathrm{M}^{2} \mathrm{~s}$ is more variable than that of the $\mathrm{M}^{1} \mathrm{~s}$ due to the reduction of the hypocone, which is responsible for 


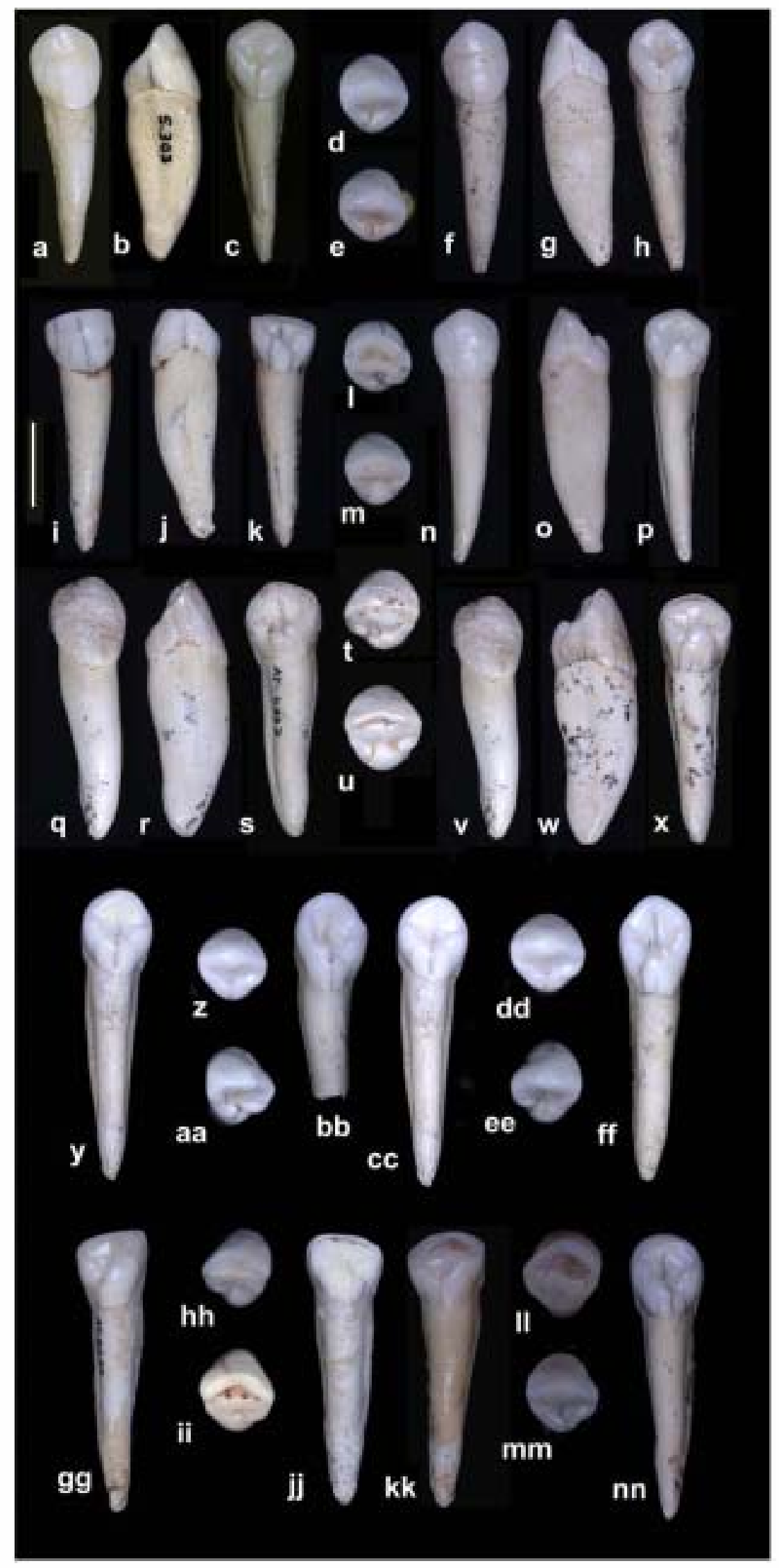

Figure 5. The SH C's. Labial (a), distal (b), lingual (c), and occlusal (d) aspect of AT-6 (L); occlusal (e), labial (f), mesial ( $\mathrm{g}$ ), and lingual (h) aspect of AT-44 (L); labial (i), mesial (j), lingual (k), and occlusal (I) aspect of AT-958 (L); occlusal (m), labial ( $\mathrm{n}$ ), mesial (o), and lingual (p) aspect of AT-1757 (L); labial (a), mesial ( $\mathrm{r})$, lingual ( $\mathrm{s}$ ), and occlusal (t) aspect of AT$4333(\mathrm{R})$; occlusal (u), labial (v), distal ( $\mathrm{w})$, and lingual ( $\mathrm{x}$ ) aspects of AT-4335 (L); lingual (y) and occlusal ( $\mathrm{z}$ ) aspect of AT-3292 (L); occlusal (aa) and lingual (bb) aspect of AT-2151 (L); lingual (cc) and occlusal (dd) aspect of AT-2392 (L); occlusal (ee) and lingual (ff) aspect of AT-558 (R); lingual (gg) and occlusal (hh) aspect of AT-3255 (R); occlusal (ii) and lingual (ij) aspect of AT- $163(\mathrm{~L})$; lingual (kk) and occlusal (II) aspect of AT-5616 (R); occlusal (mm) and lingual (nn) aspect of AT-5622 (L). L: left, R: right. White bar $=1 \mathrm{~cm}$. 

contemporary H. supiens.

\begin{tabular}{|c|c|c|c|c|c|c|}
\hline$C^{\prime}$ & Grade & SH & HEI & NEA & FSAP & SAP \\
\hline \multirow[t]{8}{*}{ Tuberculum dentale } & 0 & 0 & $1(33.3 \%)$ & 0 & $2(20 \%)$ & $64(56.6 \%)$ \\
\hline & 1 & $2(9.5 \%)$ & $1(33.3 \%)$ & 0 & $2(20 \%)$ & $25(22.1 \%)$ \\
\hline & 2 & $4(19 \%)$ & 0 & 0 & $2(20 \%)$ & $14(12.4 \%)$ \\
\hline & 3 & $6(28.6 \%)$ & $1(33.3 \%)$ & $6(28.6 \%)$ & $2(20 \%)$ & $9(8 \%)$ \\
\hline & 4 & $2(9.5 \%)$ & 0 & $5(23.8 \%)$ & $2(20 \%)$ & 0 \\
\hline & 5 & $3(14.3 \%)$ & 0 & $7(33.3 \%)$ & 0 & 0 \\
\hline & 6 & $4(19 \%)$ & 0 & $3(14.3 \%)$ & 0 & $1(0.9 \%)$ \\
\hline & Total & 21 & 3 & 21 & 10 & 113 \\
\hline \multirow[t]{7}{*}{ Shovel shape } & 0 & 0 & 0 & 0 & 0 & $30(26.8 \%)$ \\
\hline & 1 & 0 & 0 & 0 & $2(22.2 \%)$ & $37(33 \%)$ \\
\hline & 2 & 0 & 0 & 0 & $4(44.4 \%)$ & $23(20.5 \%)$ \\
\hline & 3 & $4(18.2 \%)$ & $1(33.3 \%)$ & $9(40.9 \%)$ & $3(33.3 \%)$ & $17(15.2 \%)$ \\
\hline & 4 & $11(50 \%)$ & $2(66.7 \%)$ & $9(40.9 \%)$ & 0 & $4(3.6 \%)$ \\
\hline & 5 & $7(31.8 \%)$ & 0 & $4(18.2 \%)$ & 0 & $1(0.9 \%)$ \\
\hline & Total & 22 & 3 & 22 & 9 & 112 \\
\hline \multirow[t]{4}{*}{ Canine mesial ridge } & 0 & $1(5.3 \%)$ & $1(50 \%)$ & $5(26.3 \%)$ & $6(66.7 \%)$ & $106(93 \%)$ \\
\hline & 1 & $3(15.8 \%)$ & $1(50 \%)$ & $6(31.6 \%)$ & $2(22.2 \%)$ & $2(1.8 \%)$ \\
\hline & 2 & $15(78.9 \%)$ & 0 & $8(42.1 \%)$ & $1(11.1 \%)$ & $6(5.3 \%)$ \\
\hline & Total & 19 & 2 & 19 & 9 & 114 \\
\hline \multirow[t]{4}{*}{ Distal accessory ridge } & 0 & $7(50 \%)$ & $1(50 \%)$ & $9(56.3 \%)$ & $2(28.6 \%)$ & $61(67.8 \%)$ \\
\hline & 1 & $2(143 \%)$ & $1(50 \%)$ & $4(25 \%)$ & $3(42.8 \%)$ & $18(20 \%)$ \\
\hline & 2 & $5(35.7 \%)$ & 0 & $3(18.8 \%)$ & $2(28.6 \%)$ & $11(12.2 \%)$ \\
\hline & Total & 14 & 2 & 16 & 7 & 90 \\
\hline
\end{tabular}

the MD shortening. The lingual side of the parallelogram is reduced, and the tooth adopts an irregular oval shape or a "heart shaped" contour (with its vertex toward lingual) when the hypocone reduction is also associated with a metacone reduction.

The protocone occupies almost the whole lingual face because of the hypocone reduction. The size of the metacone varies from moderately large (grade 4 ) in $38.9 \%$ of the sample (e.g., AT-822), to a small cusp (grade 3 ) in $\mathbf{4 4 . 4 \%}$ of cases, or even a cusplet (grade 2 ) in $16.7 \%$ (e.g., AT-815, AT-824), but never reaches the size of the protocone.

The hypocone undergoes a notable reduction when compared to the $\mathrm{M}^{1}$ s. In 33.4\% of cases, it is absent (grade 0) (e.g., AT-15, AT-588) or only detectable by the presence of a short, deep distal groove (grade 1) (e.g., AT-817). In AT-2175 and AT-2179, the hypocone is strongly displaced toward the cervical region, adopting the aspect of an accessory cusp. In $44.4 \%$ of specimens, the hypocone is a small cusp (grade 2) (e.g., individual XVIII), and only in $16.7 \%$ of specimens is it large (grade 4) (e.g., AT-407, AT-4336). None of the specimens reach the highest degree of expression (i.e., grade 5).

Only $33.4 \%$ of individuals express a C5 of moderate size (grade $\geq 3$ ) (e.g., individual $X X$ ), which is delimited by the distal bifurcation of the distal groove. In AT-2175, the C5 has a bitubercular morphology. In the remaining cases, this accessory cusp is absent $(27.8 \%)$, hinted by the bifurcation of the distal groove (grade 1 in $16.7 \%$ of cases) or expressed only as a cusplet (grade 2 in 22.2\%) (e.g., individual VII).

In almost all of the specimens examined (83.3\%), the crista obliqua is continuous. The transverse crest is only present in $23.5 \%$ of the sample (e.g., individuals VII, XII, XVI), and in more than the half of the specimens (61.5\%), we find small mesial accessory tubercles that are slightly displaced buccally.

Except for those cases where the lingual face of the protocone presents a slight depression (33.3\%) or a groove (11.1\%) that could correspond to a Carabelli's trait due to its location, this trait is absent in the sample. No parastyle is expressed.

The majority of the $\mathrm{M}^{2}$ s have three roots: one lingual and two buccal (mesial and distal). These roots are less divergent than in the $\mathrm{M}^{1} \mathrm{~s}$ and are deviated toward the distal side. Frequently, the buccal roots are fused in their cervical third (e.g., AT-817, AT-15, AT-815) and coalesce (joined by a lamina) for almost the whole length of the apical two-thirds. The buccal roots are mesiodistally compressed, while the lingual root is buccolingually compressed. The mesiobuccal root is wider than the lingual one, and in some cases (e.g., AT-407, AT-824), it presents a longitudinal groove demarcating the radicals, though it never produces a true bifurcation. In AT-822, there is a cementum and/or dentine lamina joining the lingual and the distobuccal roots.

Some degree of taurodontism is present in $73.7 \%$ of the sample. In $53.3 \%$ of cases, root division occurs from the first third of the root length (e.g., individuals III, VII, XXII), and in one individual (XVII), the root division starts in the second third of the root. In $13.3 \%$ of the sample, hypertaurodontism is observed, resulting into a monoradicular tooth (e.g., individuals IV and VII).

Upper third molar $\left(\mathrm{M}^{3}\right)$ The SH $\mathrm{M}^{3} \mathrm{~s}$ are well-preserved; however, the lingual apex of AT-171 and the apical third of the lingual root, two-thirds of the buccal roots of AT-194, two-thirds of AT-3183 root, the root of AT-5082, and the mesial aspect of the crown of AT-2135 are all missing (Fig. 12, Table 14).

The occlusal outline of the $\mathbf{M}^{3} \mathrm{~s}$ is highly variable, with several atypical conformations. It can be subtriangular or oval, with the $\mathrm{BL}$ axis being the largest. The occlusal polygon appears to be compressed, and, from the occlusal view, a considerable part of the buccal and lingual faces can be seen. The majority of the specimens have only three main cusps: the protocone, the paracone, and the metacone, which are also variable in their size and shape. The protocone is the largest and the metacone is always small, only reaching the size of a cusplet or a crest (grade 1 and 2 ) in $80 \%$ of cases, and it tends to occupy a distal position within the tooth contour. The hypocone is absent (grade 0 ) in more than half of the sample (57.1\%) (e.g., AT-602, AT-819), and, in the remaining cases, it develops either as a crest (grade 1 in 38.1\%) or a cusplet (grade 2 in $4.8 \%$ ). The C5 is not present in the sample. Only individual XI expresses a weak crest that could be identified with the lowest degree of expression of this trait. The posterior fovea is absent or obliterated by wear. From the central fovea, a short central fissure emerges separating the protocone and the paracone. Distally, a Vshaped groove delimits the metacone extension. The enamel crenulations and the secondary furrows are not very prof use, but 


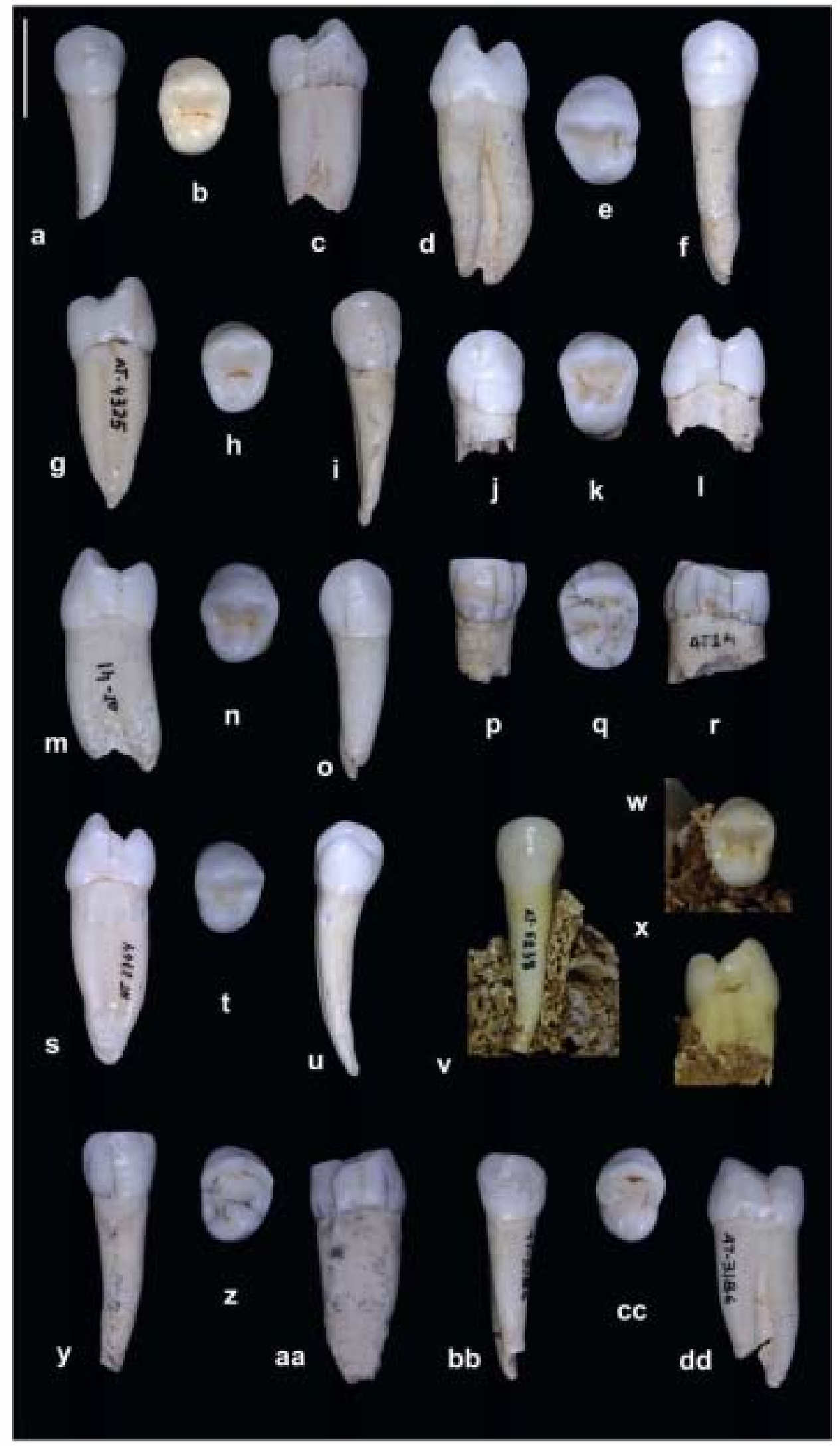

Figure 6. The SH P ${ }^{3}$ s. Buccal (a), occlusal (b), and distal (c) aspect of AT-1944 (R); distal (d), occlusal (e), and lingual (f) aspect of AT-589 (R); distal (g), occlusal (h), and buccal (i) aspect of AT-4325 (L); buccal (j), occlusal (k), and mesial (I) aspect of AT-2036 (L); mesial (m), buccal (n), and occlusal (o) aspect of AT-41 (L); occlusal (p), mesial ( $(\mathbf{q})$, and buccal (r) aspect of AT-24 (R); mesial (s), occlusal (t), and lingual (u) aspect of AT-2764 (L); buccal (v), occlusal (w), and mesial (x) aspect of AT-5838 (L); buccal (y), occlusal ( $(\mathrm{z})$, and mesial (aa) aspect of AT-2782 (L); buccal (bb), occlusal (cc), and distal (dd) aspect of AT-3186 (R). L: left, R: right. White bar $=1 \mathrm{~cm}$. 
Table 10

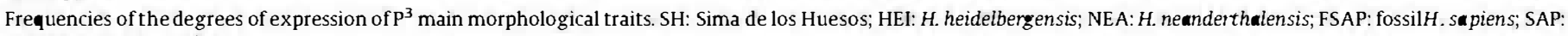
contemporary H. supiens.

\begin{tabular}{|c|c|c|c|c|c|c|}
\hline $\mathrm{P}^{3}$ & Grade & SH & HEI & NEA & FSAP & SAP \\
\hline \multirow[t]{4}{*}{ Buccal essential crest } & 0 & $8(47.1 \%)$ & 0 & $1(6.7 \%)$ & 0 & $1(0.8 \%)$ \\
\hline & 1 & $5(29.4 \%)$ & $1(50 \%)$ & $6(40 \%)$ & $3(75 \%)$ & $111(88.1 \%)$ \\
\hline & 2 & $4(23.5 \%)$ & $1(50 \%)$ & $8(53.3 \%)$ & $1(25 \%)$ & $14(11.1 \%)$ \\
\hline & Total & 17 & 2 & 15 & 4 & 126 \\
\hline \multirow[t]{4}{*}{ Lingual essential crest } & 0 & 0 & 0 & 0 & 0 & $1(0.8 \%)$ \\
\hline & 1 & $17(89.5 \%)$ & 0 & $5(33.3 \%)$ & $3(75 \%)$ & $118(95.2 \%)$ \\
\hline & 2 & $2(10.5 \%)$ & $2(100 \%)$ & $10(66.7 \%)$ & $1(25 \%)$ & $5(4 \%)$ \\
\hline & Total & 19 & 2 & 15 & 4 & 124 \\
\hline \multirow[t]{4}{*}{ Transverse crest } & 0 & $13(68.4 \%)$ & $3(100 \%)$ & $14(93.3 \%)$ & $4(80 \%)$ & $128(100 \%)$ \\
\hline & 1 & $5(26.3 \%)$ & 0 & $1(6.7 \%)$ & $1(20 \%)$ & 0 \\
\hline & 2 & $1(5.3 \%)$ & 0 & 0 & 0 & 0 \\
\hline & Total & 19 & 3 & 15 & 5 & 128 \\
\hline \multirow[t]{3}{*}{ Distal accessory ridge } & 0 & $10(71.4 \%)$ & $1(50 \%)$ & $7(63.3 \%)$ & $1(50 \%)$ & $94(89.5 \%)$ \\
\hline & 1 & $4(28.6 \%)$ & $1(50 \%)$ & $4(36.4 \%)$ & $1(50 \%)$ & $11(10.5 \%)$ \\
\hline & Total & 14 & 2 & 11 & 2 & 105 \\
\hline \multirow[t]{3}{*}{ Mesial accessory ridge } & 0 & $14(100 \%)$ & $1(50 \%)$ & $10(90.9 \%)$ & $2(100 \%)$ & $92(86 \%)$ \\
\hline & 1 & 0 & $1(50 \%)$ & $1(9.1 \%)$ & 0 & $15(14 \%)$ \\
\hline & Total & 14 & 2 & 11 & 2 & 107 \\
\hline
\end{tabular}

they can make it difficult to identify the main cusps in some cases (e.g., AT-274, AT-3181). No crista obliqua is expressed, and a transverse crest is present in only one case (individual XII).

In 20\% of the $\mathrm{SH} \mathrm{M}^{3} \mathrm{~s}$, a Carabelli's tubercle can be observed, ranging from a slight depression (grade 1) in AT-601, to a more pronounced depression in AT-2393, and a clear Y-shaped depression in AT-945. With only the exception of AT-805, the parastyle is absent in the sample.

The radical system of this tooth type is frequently fused. Half of the sample bears three roots: one lingual, one mesiobuccal, and one distobuccal that all diverge distally. Commonly, these roots are fused or joined by a thick lamina (e.g., AT-816, AT-171), and this is particularly true of the buccal roots. In AT-951, the lingual root is also joined by a lamina. The mesiobuccal root is mesiodistally compressed and is wider than the distobuccal root, with a longitudinal groove in some cases (e.g., AT-819).

In some specimens, the three roots are totally fused (e.g., AT-10, AT-140). One-third of the sample exhibits two roots, and AT-4102 is mono-radicular. In more than half of the sample, the root division occurs from the first third, but without internal images, and considering the high number of root fusions we observed, it is difficult to certify the existence of taurodontism. In addition, many of the specimens in the sample belong to young individuals in which the root system is not complete.

Lower central incisors $\left(\mathrm{I}_{1}\right)$ The $\mathrm{SH} \mathrm{I}_{1} \mathrm{~S}$ are generally well-preserved, but the distal third of the roots of AT-2384 and AT-3242, and the apex of AT-3241, AT-2730, and AT-162 are all broken. AT-4 only preserves the proximal third of the root and AT-956 exhibits superficial enamel loss in the labial aspect (Fig. 13, Table 15).

From the buccal aspect, the crown shows a spatulate shape in the form of a triangle, with the vertex toward the cemento-enamel junction and the base at the incisal edge. The crown is generally symmetrical, though the mesial angle of the incisal border tends to be sharper than the distal one.

The lingual surface is generally smooth (e.g., AT-1742), showing a slight basal swelling (grade 1 ) in only $26.3 \%$ of cases (e.g., AT-1460, AT-609) that forms a slender basal eminence without clear margins. None of the $I_{1} s$ present tuberculum dentale, lingual fossae, or pronounced marginal ridges (AT-1742, AT-162). There are clear traces of shovel shape (grade 2) in only two cases (individuals XVIII and XX).

From the incisal aspect, the incisal edge shows a basically symmetric curvature. The labial convexity is slight (grade 2) in about half of the sample (52.6\%), moderate (grade 3 ) in $42.1 \%$, and only one specimen exhibits a pronounced labial curvature (AT-3241).

Lateral aspects of the tooth are similar, though the cementoenamel line is lower and less curved on the distal side than on the proximal one. From this aspect, the $\mathrm{SH}_{1} \mathrm{~s}$ look robust and wide despite their size.

The root is single, with an oval section that is mesiodistally compressed. A wide and shallow longitudinal groove runs along the distal and mesial surface of the root, being more pronounced on the latter. The root is essentially straight, though it tends to deviate slightly distally.

Lower lateral incisors $\left(\mathrm{I}_{2}\right)$ The $\mathrm{SH} \mathrm{I}_{2} \mathrm{~S}$ are generally well-preserved, with the exception of some small fractures at the apex in AT-597, AT-55, and AT-167, in the apical third of the root of AT-103 and AT-723, in the distal two-thirds of AT- 1726 and AT-608, and with some superficial enamel loss on the labial surface of AT-2066 (Fig. 14, Table 16).

From the labial aspect, the crown has a trapezoid contour with its wider base toward the incisal edge. The crown is less symmetrical than in the $I_{1} s$, with the mesial margin being slightly longer than the distal one, and the mesial incisal angle being sharper than the distal.

In $73.7 \%$ of cases, the $I_{2} S$ show a basal eminence on the lingual surface (grade 1 of tuberculum dentale), and this swelling is separated from the essential ridge by a small depression (grade 2 ) in only one case (AT-1123). The shovel shape is also weak in its manifestation in these teeth, with $42.1 \%$ of specimens showing traces of shovel shape (grade 2) (e.g., AT-597, AT-103) and only $21.1 \%$ expressing moderate shovel shape.

From the incisal aspect, $47.4 \%$ of the sample shows moderate convexity (grade 3), while the convexity expression is weak (grade 2 ) in $52.6 \%$ of the sample. The incisal edge is more asymmetrical than in the $\mathrm{I}_{1} \mathrm{~S}$, and the distal incisal edge has a clearly more lingual position than the mesial edge.

Lateral aspects of the teeth are similar, though the cementoenamel junction is higher on the distal side. The interproximal wear facet always occupies the incisal third of the crown, though this facet is deviated labially as a result of anterior crowding of the teeth in individual XVIII.

The root in this tooth type is single, with an oval section that is mesiodistally compressed with a slight distal deviation of its apical third. A shallow longitudinal groove runs along the root length on both the mesial and distal sides. 


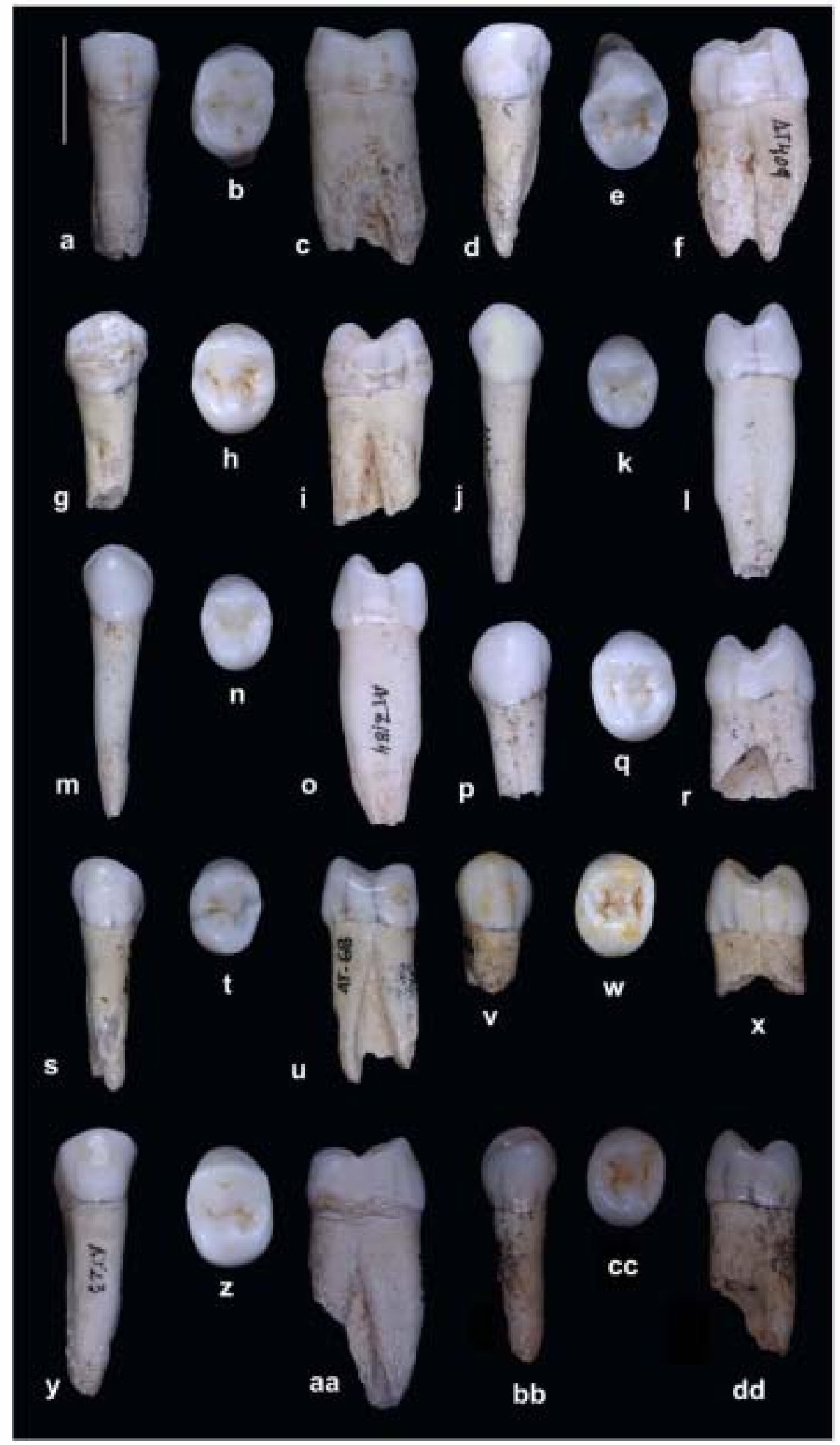

Figure 7. The SH P4. Buccal (a), occlusal (b), and distal (c) aspect of AT-193 (R); buccal (d), occlusal (e), and distal (f) aspect of AT-409 (L); buccal (g), occlusal (h), and distal (i) aspect of AT-559 (L); buccal $(\mathrm{j})$, occlusal $(\mathrm{k})$, and mesial (I) aspect of AT-949 $(\mathrm{R})$; lingual $(\mathrm{m})$, occlusal $(\mathrm{n})$, and mesial $(\mathrm{o})$ aspect of AT-3184 (L); buccal (p), occlusal $(\mathbf{q})$, and distal $(\mathrm{r})$ aspect of AT-2070 (L); buccal (s), occlusal ( $(\mathrm{t})$, and distal (u) aspect of AT-68 (R); buccal (v), occlusal (w), and distal ( $\mathrm{x}$ ) aspect of AT-746; buccal (y), occlusal (z), and distal (aa) aspect of AT-23; lingual (bb), occlusal (cc), and distal (dd) aspect of AT-5510 (R). L: left, R: right. White bar $=1 \mathrm{~cm}$. 

contemporary H. supiens.

\begin{tabular}{|c|c|c|c|c|c|c|}
\hline $\mathrm{P}^{4}$ & Grade & SH & HEI & NEA & FSAP & SAP \\
\hline \multirow[t]{4}{*}{ Buccal essential crest } & 0 & 0 & 0 & 0 & 0 & $1(0.9 \%)$ \\
\hline & 1 & $12(66.7 \%)$ & $3(50 \%)$ & $5(33.3 \%)$ & $7(63.6 \%)$ & $95(89.6 \%)$ \\
\hline & 2 & $6(33.3 \%)$ & $3(50 \%)$ & $10(66.6 \%)$ & $4(36.4 \%)$ & $10(9.4 \%)$ \\
\hline & Total & 18 & 6 & 15 & 11 & 106 \\
\hline \multirow[t]{4}{*}{ Lingual essential crest } & 0 & 0 & 0 & 0 & 0 & 0 \\
\hline & 1 & $9(50 \%)$ & $2(33.3 \%)$ & $3(21.4 \%)$ & $11(100 \%)$ & $99(91.7 \%)$ \\
\hline & 2 & $9(50 \%)$ & $4(66.7 \%)$ & $11(78.6 \%)$ & 0 & $9(8.3 \%)$ \\
\hline & Total & 18 & 6 & 14 & 11 & 108 \\
\hline \multirow[t]{4}{*}{ Transverse crest } & 0 & $15(78.9 \%)$ & $7(100 \%)$ & $14(87.5 \%)$ & $9(81.8 \%)$ & $113(100 \%)$ \\
\hline & 1 & $4(21.1 \%)$ & 0 & $1(6.2 \%)$ & $1(9.1 \%)$ & 0 \\
\hline & 2 & 0 & 0 & $1(6.2 \%)$ & $1(9.1 \%)$ & 0 \\
\hline & Total & 19 & 7 & 16 & 11 & 113 \\
\hline \multirow[t]{3}{*}{ Distal accessory ridge } & 0 & $7(53.8 \%)$ & $1(20 \%)$ & $7(58.3 \%)$ & $4(44.4 \%)$ & $70(79.5 \%)$ \\
\hline & 1 & $6(46.2 \%)$ & $4(80 \%)$ & $5(41.7 \%)$ & $5(55.6 \%)$ & $18(20.5 \%)$ \\
\hline & Total & 13 & 5 & 12 & 9 & 88 \\
\hline \multirow[t]{3}{*}{ Mesial accessory ridge } & 0 & $7(53.8 \%)$ & $3(50 \%)$ & $8(88.8 \%)$ & $1(12.5 \%)$ & $74(80.4 \%)$ \\
\hline & 1 & $6(46.2 \%)$ & $3(50 \%)$ & $1(11.2 \%)$ & $7(87.5 \%)$ & $18(19.6 \%)$ \\
\hline & Total & 13 & 6 & 9 & 8 & 92 \\
\hline
\end{tabular}

Lower canines (C.) The state of preservation of the $\mathrm{SH} \mathrm{C,S} \mathrm{is}$ generally good. There is a superficial loss of enamel on the labial aspect of AT-578. The distal third of the roots of AT-60, AT-67, and AT-2783, and the distal two-thirds of the roots of AT-808, are missing (Fig. 15, Table 17). There is one specimen, AT-1144, that has not been included in this analysis because the root and crown are pathologically deformed.

The crowns of these teeth exhibit an incisor-like aspect. The shape is trapezoidal/rectangular due to the wear of the crown tip. When the tip is unworn or almost unworn, the contour is pentagonal, with the mesial arm of the incisal edge slightly shorter than the distal arm. The lingual aspect is smooth, and the essential ridge is not expressed. The distal marginal ridge is generally shorter than the mesial one, with its length being restricted to the lower third of the crown, and, in some cases, adopting the aspect of a tubercle (e.g., AT-1952). The marginal ridges are thickened, ranging from minimal shovel expression (grades 1 and 2) in approximately 21\% of specimens (e.g., individuals XXV and XVI) to moderate and marked degrees of shovel expression (grade $\geq 3$ ) in approximately $80 \%$ of the sample (e.g., individuals VII and XXIII). The distal marginal ridge is generally thicker than the mesial one, generating an asymmetric shovel shape that can range from slight to pronounced (e.g., in AT-1755, the mesial ridge is barely visible whereas the distal one is marked). In $25 \%$ of specimens, a weak distal accessory ridge is present between the essential ridge and the distal marginal ridge (e.g., AT-2, AT-567). No tuberculum dentale is expressed in any of the specimens, and in only $36.8 \%$ of the teeth, there is a mild and round basal eminence (grade 1) without demarcation from the marginal ridges. There are no signs of buccal cingulum.

The root is single and robust with an oval section that is mesiodistally compressed. From the lateral aspect, a shallow longitudinal depression running along the root, which is less marked on the distal side, can be identified. In AT-2438, the groove is deep and demarcates two clear radicals (one lingual and one buccal), but the fracture of the apical ends does not allow us to determine whether or not a bifurcation of the root existed. The cemento-enamel line tends to be rectilinear on the distal side and convex on the mesial one. The root is also rectilinear with a slight distal deviation in its apical third.

Lower third premolar $\left(\mathrm{P}_{3}\right)$ The general state of preservation of this tooth type is excellent. AT-563, AT-3045, AT-807, and AT-2027 all have a small fracture of the root apex. AT-3243 lacks one-third of its root, and AT-74 and AT-3243 lack two-thirds of their roots. The crown of AT-64 is broken in its distal half (Fig. 16, Table 18).

From the occlusal aspect, the $\mathrm{P}_{3}$ s present a rounded, rhomboidal contour where the lingual half is smaller than the buccal. With only one exception (AT-792), the SH P3s have both main cusps. The buccal cusp, and particularly its buccal surface, is significantly larger than the lingual cusp and strongly projected onto the occlusal plane so that its tip is centered and close to the tip of the lingual cusp. Thus, the occlusal polygon is small (see Gómez-Robles et al., 2008) and occupies a lingual position within the tooth contour. The lingual cusp is also small and has a globular aspect that is usually well-demarcated by a short sagittal groove that can extend to the lingual border of the tooth and cross it, particularly at its mesial end. In a high proportion of the specimens in the sample (83.3\%), both main cusps are connected by a transverse crest of varying thickness (grade 2 ). In AT-1780, this crest is conspicuous and it occupies a mesial position, whereas it deviates distally in AT2768 and AT-1466. In approximately half of the sample (53.3\%), the buccal cusp expresses a distal accessory ridge, and the mesial accessory ridge is almost absent (6.7\%). The anterior and posterior foveae tend to be pit-like and small, and, in some cases, they do not develop at all (e.g., AT-2438). The tips of the main cusps are generally centered; however, in a small percentage of cases, they are slightly deviated mesially (27\%; e.g., AT-148, AT-2) or distally (22\%; e.g., AT-2767, AT-3940). In 23.5\% of the $\mathrm{P}_{3}$ S, only the metaconid is present, and in $52.9 \%$ of individuals, apart from the metaconid, there is a small distolingual platform without a free apex. Three individuals present an accessory cusp and AT-300 is the only case where we can identify two accessory lingual cusps plus the metaconid.

All of the traits that are explained above contribute to the canine-like aspect of the $\mathrm{SH} \mathrm{P}_{3}$ s. In individuals XVIII and XXV, the flatness of the essential buccal ridge makes this canine-like aspect even more pronounced.

From the lateral aspect, there is a strong lingual inclination of the buccal aspect of the crown. There are no signs of buccal cingulum, but a basal swelling can be observed in all these teeth.

The root is single and mesiodistally compressed. In $75 \%$ of cases, we determined the presence of a developmental depression that runs longitudinally from approximately $3-4 \mathrm{~mm}$ from the cemento-enamel junction to the apex, which is deeper on the mesial side but never exceeds a grade 3 of Tomes' root (according to Turner et al.'s [1991] classification). The roots are generally straight 


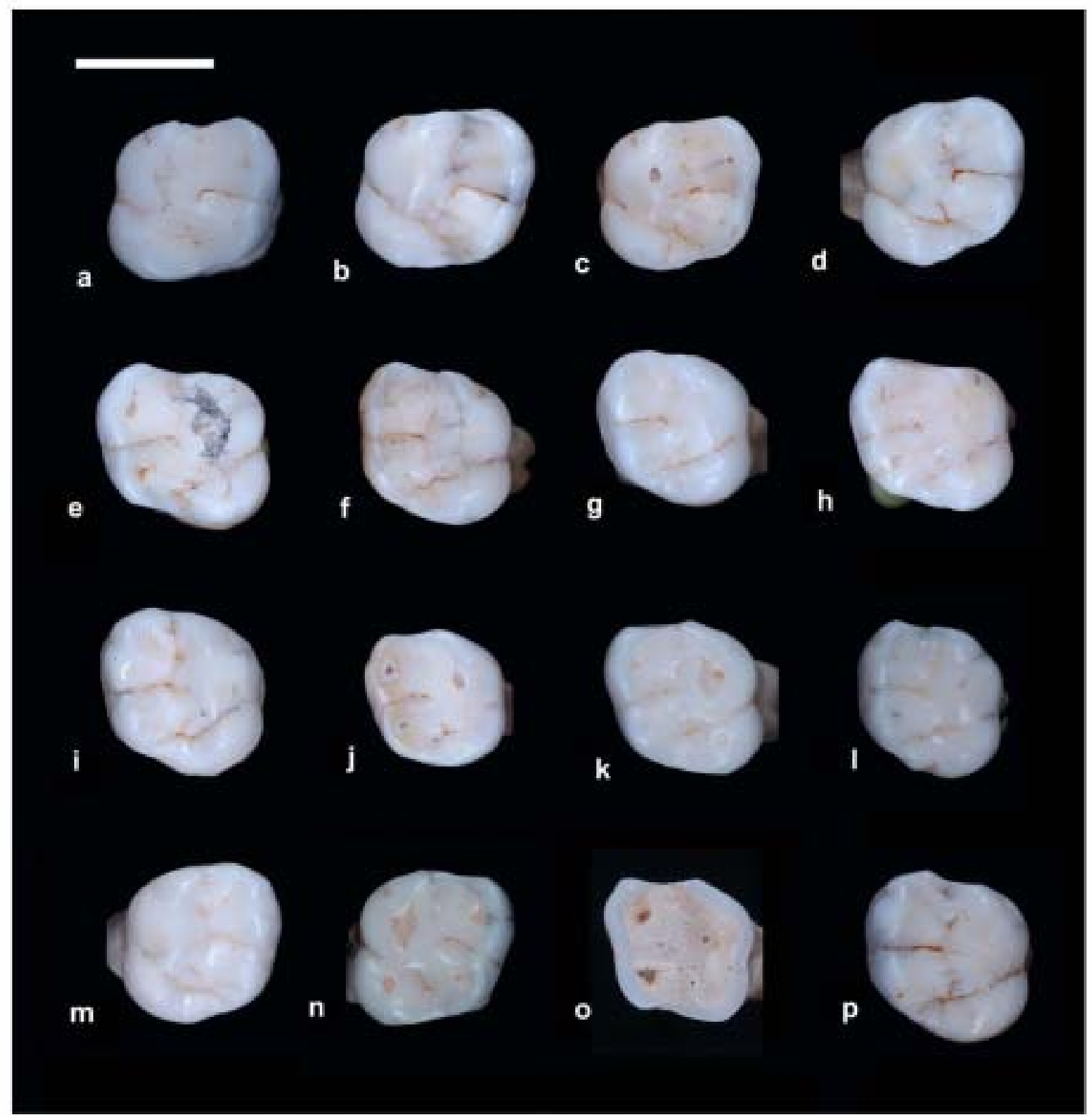

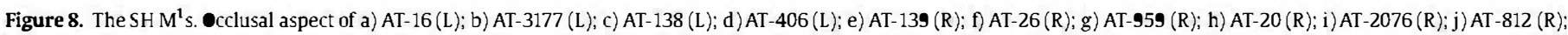

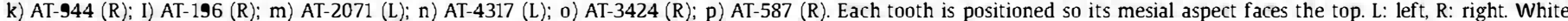
bar $=1 \mathrm{~cm}$

with a mild distal curvature from the medial third to the apex. Although all of the $\mathrm{P}_{3} \mathrm{~S}$ in the SH sample are single-rooted, we note that the mandible fragment, labeled as AT-172, has two radicals in situ in the $\mathrm{P}_{3}$ socket, one of which is mesiobuccal and one of which is distolingual.

Lower fourth premolar $\left(\mathrm{P}_{4}\right)$ The $\mathrm{SH}_{4} \mathrm{~S}$ are very well-preserved, with the small exceptions of AT-28, AT-806, AT-3188, AT-2275, and AT-1467, in which the distal extreme of the root is broken off, and AT-2386 and AT-828, in which the distal two-thirds are missing (Fig. 17, Table 19). In AT-300, the tooth crowns show a generalized pitting defect of the enamel.

From the occlusal aspect, the contour is an asymmetric oval with a distolingual bulge due to the expression of a talonid (e.g., AT-9, AT-147, AT-28) that provides these teeth with their characteristic shape. All of these teeth have the two main cusps, and, with isolated exceptions (AT-3939, AT-AT-147, and AT-28), all of them develop from one (individual XXII) to two (e.g., individuals I, II, IV, VI, and VII; 90\%) or three (individual XII) accessory cusps.

The buccal cusp is centered or mesially displaced with respect to the occlusal contour. The lingual cusp is smaller and tends to occupy a mesial position relative to both the tip of the buccal cusp and the contour of the tooth. The expression of the extralingual cusps may contribute to the asymmetric contour of the tooth and may also contribute to the mesial displacement of the metaconid.

With the exception of individual IV and one of the antimeres of individuals XXV, XXIII, VII, and III, all of the specimens present a transverse crest connecting the buccal and the lingual main cusps. In $75 \%$ of individuals, this crest is conspicuous and/or continuous (grade 2), while in 20\% of individuals, it is weak and/or interrupted by the sagittal groove (grade 1 ). In all cases, this crest has a mesial 


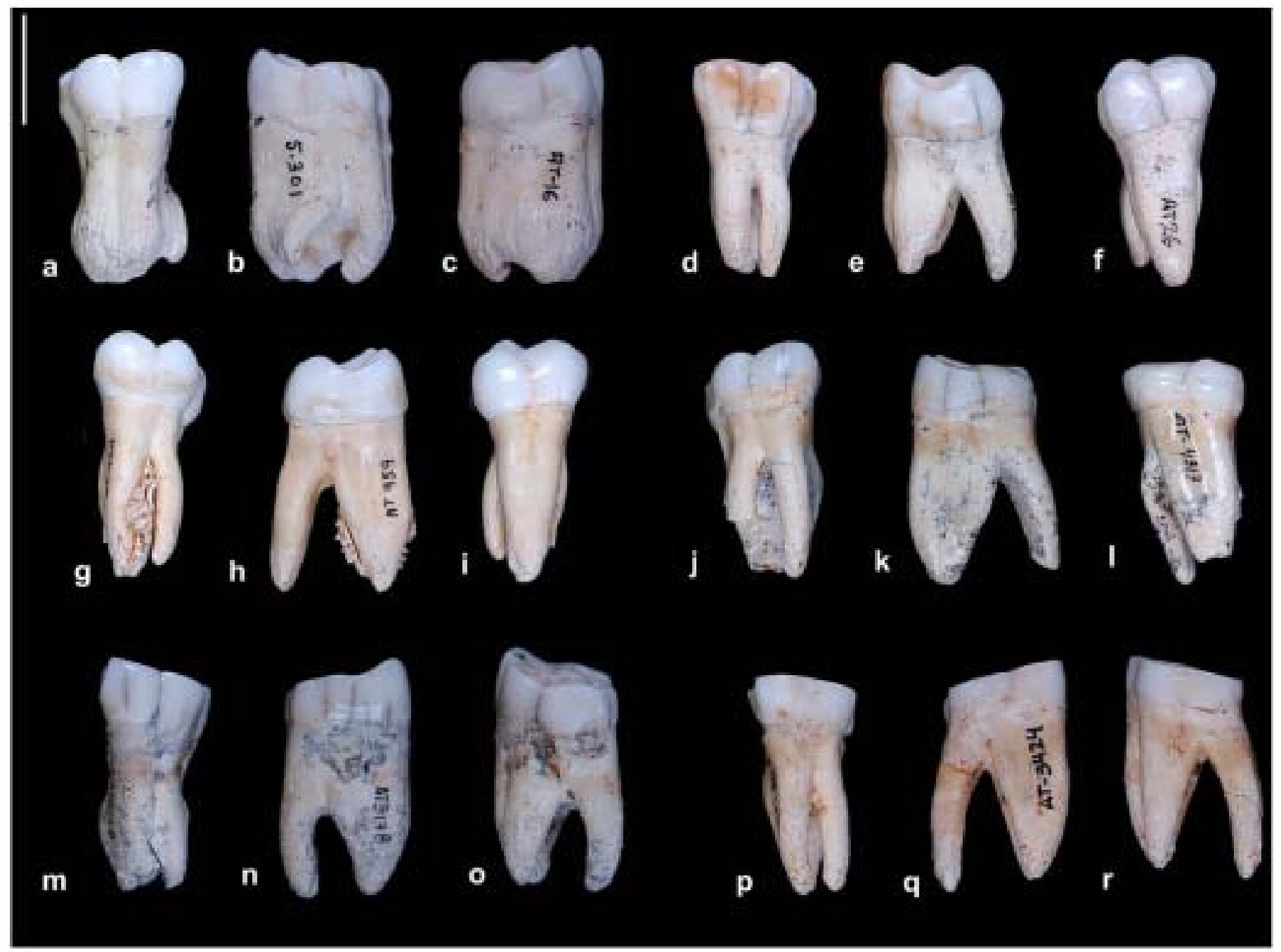

Figure 9. The SH M ${ }^{1}$ s. Buccal (a), mesial (b), and distal (c) aspect of AT-16 (L); buccal (d), distal (e), and lingual (f) aspect of AT-26 (R); buccal (g), mesial (h), and lingual (i) aspect of

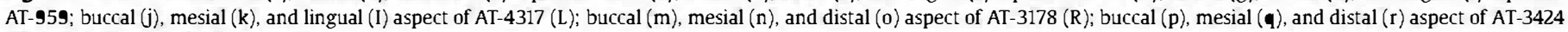
(R). L: left, R: right. White bar $=1 \mathrm{~cm}$.

position, erasing the mesial end of the sagittal groove when it is continuous.

The anterior fovea is shorter and shallower than the posterior fovea and it may be reduced to a pit. In some cases, the posterior fovea presents a star-like aspect (e.g., AT-2245) due to the radiation of the accessory grooves that delimit the accessory cusps in the talonid. In $66.7 \%$ of cases, the protoconid expresses a distal accessory ridge arising from the posterior fovea(e.g., AT-277, AT-3187, AT-608, AT-1467), but no mesial accessory ridge is found in any case.

In lateral view, there is no cingulum but the existence of a basal swelling and lingual inclination of the buccal surface is observed.

The root is single and it is mesiodistally compressed, exhibiting a wide but shallow groove along the distal surface. The direction of the root is straight with a slight distal deviation of the root apex. Lower first molar $\left(\mathrm{M}_{1}\right)$ This tooth type is extraordinarily wellpreserved in the sample with only some minor defects, such as the apical two-thirds of the roots of AT-175, AT-1459, AT-141, AT576, AT-56, the distal third of AT-2276, and the distal two-thirds of the mesial root of AT-286. In AT-43, the lingual part of the crown and upper third of the root is missing, and AT-272 lacks the whole root system (Fig. 18, Table 20).

The occlusal contour of the $\mathrm{SH} \mathrm{M}_{1}$ s has the shape of an oval or rounded rectangle that is mesiodistally elongated. Almost all of the
$\mathrm{M}_{1}$ s have the five main cusps, and the extension of each cusp is only slightly, if at all, marked on the external contour of the tooth. All the $\mathrm{M}_{1}$ s present an anterior fovea that is mesially bounded by the mesial marginal ridge and is distally bounded by the protoconid and the metaconid. In $85 \%$ of cases, this fovea has the shape of a short, deep fissure or a deep pit (grade 2). In the remaining cases, this fovea is lineal or superficial (grade 1). With isolated exceptions (AT-2193, AT-943, AT-605), the 95\% of SH individuals present a continuous middle trigonid crest that is expressed as a conspicuous enamel bridge and erases the sagittal groove before reaching the anterior fovea. The association of the pit-like anterior fovea and the middle trigonid crest is a consistent throughout the SH sample. The mesial marginal ridge is easily affected by occlusal wear, but when it is unworn it is possible to identify the expression of marginal accessory tubercles (e.g., AT-3175).

In the metaconid, the essential ridge delimits two adjacent plateaus, one of which is mesial and one of which is distal. In $15 \%$ of individuals, the distal plateau is delimited by two weak lingual grooves forming a C7 in its lower degrees of expression (grade 1). The $\mathrm{C} 7$ is absent in half of the sample but is present as a distinct cusp of moderate (grade 3 ) or large size (grade 4 ) in $15 \%$ and $5 \%$ of individuals, respectively. In some cases, it invades the space of the metaconid and the hypoconid (e.g., individuals VII and XXII). In 
Table 12

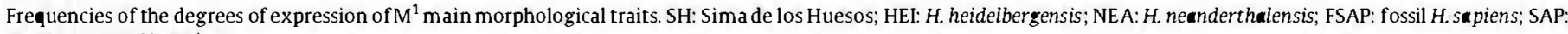
contemporary $H$. sapiens.

\begin{tabular}{|c|c|c|c|c|c|c|}
\hline $\mathrm{M}^{1}$ & Grade & $\mathrm{SH}$ & HEI & NEA & FSAP & SAP \\
\hline \multirow[t]{7}{*}{ Metacone size } & 0 & 0 & 0 & 0 & 0 & 0 \\
\hline & 1 & 0 & 0 & 0 & 0 & 0 \\
\hline & 2 & 0 & 0 & 0 & 0 & $1(0.8 \%)$ \\
\hline & 3 & $1(5.9 \%)$ & 0 & $6(26.1 \%)$ & $5(26.3 \%)$ & $21(16.5 \%)$ \\
\hline & 4 & $14(82.4 \%)$ & $4(100 \%)$ & $13(56.5 \%)$ & $11(57.9 \%)$ & $73(57.5 \%)$ \\
\hline & 5 & $2(11.8 \%)$ & 0 & $4(17.4 \%)$ & $3(15.8 \%)$ & $32(25.2 \%)$ \\
\hline & Total & 17 & 4 & 23 & 19 & 127 \\
\hline \multirow[t]{7}{*}{ Hypocone size } & 0 & 0 & 0 & 0 & 0 & $1(0.8 \%)$ \\
\hline & 1 & 0 & 0 & 0 & 0 & 0 \\
\hline & 2 & 0 & 0 & 0 & $1(5.3 \%)$ & $2(1.6 \%)$ \\
\hline & 3 & $1(5.9 \%)$ & 0 & $1(4.3 \%)$ & $2(10.5 \%)$ & $11(8.7 \%)$ \\
\hline & 4 & $5(29.4 \%)$ & $4(80 \%)$ & $10(43.5 \%)$ & $10(52.6 \%)$ & $58(45.7 \%)$ \\
\hline & 5 & $11(64.7 \%)$ & $1(20 \%)$ & $12(52.2 \%)$ & $6(31.6 \%)$ & $55(43.3 \%)$ \\
\hline & Total & 17 & 5 & 23 & 19 & 127 \\
\hline \multirow[t]{6}{*}{ C5 size } & 0 & $4(25 \%)$ & $1(20 \%)$ & $1(4.5 \%)$ & $1(5.6 \%)$ & $44(35.2 \%)$ \\
\hline & 1 & $3(18.8 \%)$ & 0 & $4(18.2 \%)$ & $1(5.6 \%)$ & $29(23.2 \%)$ \\
\hline & 2 & $3(18.8 \%)$ & $1(20 \%)$ & $10(45.5 \%)$ & $7(38.9 \%)$ & $37(29.6 \%)$ \\
\hline & 3 & $4(25 \%)$ & $2(40 \%)$ & $5(22.7 \%)$ & $7(38.9 \%)$ & $12(9.6 \%)$ \\
\hline & 4 & $2(12.5 \%)$ & $1(20 \%)$ & $2(9.1 \%)$ & $2(11.1 \%)$ & $3(2.4 \%)$ \\
\hline & Total & 16 & 5 & 22 & 18 & 125 \\
\hline \multirow[t]{3}{*}{ Crista obliqua } & 0 & 0 & 0 & $1(4.5 \%)$ & $3(15.8 \%)$ & $7(5.7 \%)$ \\
\hline & 1 & 15 (100\%) & $5(100 \%)$ & $21(95 \%)$ & $16(84.2 \%)$ & $116(94.3 \%)$ \\
\hline & Total & 15 & 5 & 22 & 19 & 123 \\
\hline \multirow[t]{3}{*}{ Transverse crest } & 0 & $7(58.3 \%)$ & $1(20 \%)$ & $15(75 \%)$ & $9(69.2 \%)$ & $43(54.4 \%)$ \\
\hline & 1 & $5(41.7 \%)$ & $4(80 \%)$ & $5(25 \%)$ & $4(30.8 \%)$ & $36(45.6 \%)$ \\
\hline & Total & 12 & 5 & 20 & 13 & 79 \\
\hline \multirow[t]{3}{*}{ Marginal accessory tubercles } & 0 & $4(50 \%)$ & $2(50 \%)$ & $7(36.8 \%)$ & $5(41.7 \%)$ & $51(65.4 \%)$ \\
\hline & 1 & $4(50 \%)$ & $2(50 \%)$ & $12(63.2 \%)$ & $7(58.3 \%)$ & $27(34.6 \%)$ \\
\hline & Total & 8 & 4 & 19 & 12 & 78 \\
\hline \multirow[t]{9}{*}{ Carabelli's complex } & 0 & $7(43.8 \%)$ & $1(25 \%)$ & $4(20 \%)$ & $10(62.5 \%)$ & $91(73.4 \%)$ \\
\hline & 1 & $9(56.3 \%)$ & $1(25 \%)$ & $6(30 \%)$ & $3(18.8 \%)$ & $5(4 \%)$ \\
\hline & 2 & 0 & $1(25 \%)$ & $2(10 \%)$ & $1(6.3 \%)$ & $7(5.6 \%)$ \\
\hline & 3 & 0 & 0 & $5(20 \%)$ & $2(12.5 \%)$ & $6(4.8 \%)$ \\
\hline & 4 & 0 & $1(25 \%)$ & $1(5 \%)$ & 0 & $2(1.6 \%)$ \\
\hline & 5 & 0 & 0 & 0 & 0 & $3(2.4 \%)$ \\
\hline & 6 & 0 & 0 & $1(5 \%)$ & 0 & $5(4 \%)$ \\
\hline & 7 & 0 & 0 & $1(5 \%)$ & 0 & $5(4 \%)$ \\
\hline & Total & 16 & 4 & 20 & 16 & 124 \\
\hline \multirow[t]{8}{*}{ Parastyle } & 0 & $12(70.6 \%)$ & $4(100 \%)$ & $14(70 \%)$ & $15(100 \%)$ & $121(98.4 \%)$ \\
\hline & 1 & $5(29.4 \%)$ & 0 & $3(15 \%)$ & 0 & $1(0.8 \%)$ \\
\hline & 2 & 0 & 0 & $1(5 \%)$ & 0 & $1(0.8 \%)$ \\
\hline & 3 & 0 & 0 & $2(10 \%)$ & 0 & 0 \\
\hline & 4 & 0 & 0 & 0 & 0 & 0 \\
\hline & 5 & 0 & 0 & 0 & 0 & 0 \\
\hline & 6 & 0 & 0 & 0 & 0 & 0 \\
\hline & Total & 17 & 4 & 20 & 15 & 123 \\
\hline
\end{tabular}

a low percentage of individuals in the sample, the essential ridge of the metaconid is slightly (21.1\%; individuals I, II, XI, and XXVIII) to clearly deflected (10.5\%; individuals VI and XX), so that the metaconid contacts the entoconid. The protoconid and the metaconid are also joined by a strong and/or continuous distal trigonid crest (grade 2) in 25\% of individuals (e.g., individuals XIV and XX).

The hypoconulid occupies a central and sometimes slightly buccal position on the distal margin of the tooth. It is located between the entoconid and the hypoconid, which, with the exception of cases where the $\mathrm{C} 7$ is pronounced, are of similar size and shape. The hypoconulid is a cusp of moderate to large size (grade $\geq 3$ ) in all cases, with the noticeable exception of individuals IV and XXVI, where it is totally absent. In these two individuals, the contour of the teeth is more rectangular, which is more typical of four-cusped molars, and the trajectory of the central groove in the portion that separates the entoconid and the hypoconid runs straight toward the distal margin of the tooth. We have discarded that these four-cusped molars are $M_{2}$ s instead of $M_{1} s$, since we also preserve the $\mathrm{M}_{1} \mathrm{~s}$ of the individuals they belong to. None of the $\mathrm{SH}$ $\mathrm{M}_{1} \mathrm{~s}$ express a $\mathrm{C6}$.
While 55.6\% of individuals in the sample show a Y-groove pattern, we find a "+" (e.g., individuals XII and XV) or an " $X$ " pattern (e.g., individual XXVI) in $44.4 \%$ of cases.

From the buccal aspect, no signs of cingulum are detected, and, in $33.3 \%$ of the population, the buccal groove separating the protoconid and the entoconid ends in a small depression or pit; no other examples of protostylid expression are found in this tooth class.

All of the SH $\mathrm{M}_{1}$ s present two roots, one of which is mesial and one of which is distal. These roots are wide, mesiodistally compressed, and have marked developmental grooves that are particularly pronounced in the mesial root and that mark the existence of two radicals (one mesiobuccal and one mesiolingual) that do not bifurcate in any case. The mesial root tends to be bulkier than the distal, though they are of similar length (with the exception of AT-1458 and AT-2438, where the distal root is longer).

In $31.3 \%$ of individuals, root bif urcation occurs beyond one-third of the root length. The morphology of the two apical thirds is highly variable. For example, in AT-43, the roots are curved and are strongly distally divergent. The mesial aspects of AT-3933 and AT- 


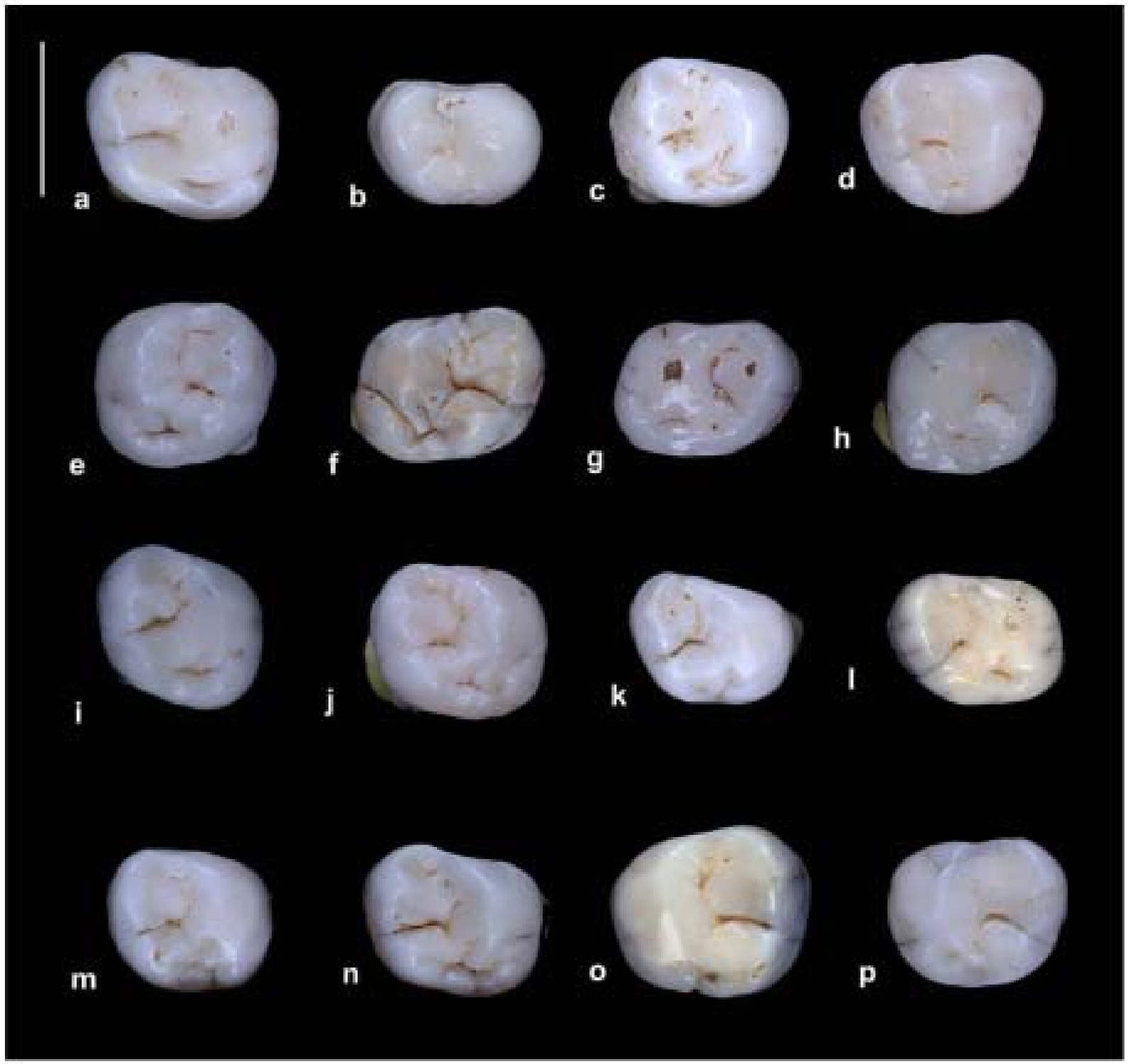

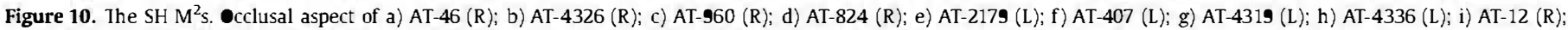

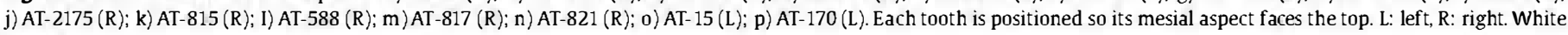
bar $=1 \mathrm{~cm}$.

3934 roots show a strong convexity. Other specimens present less divergent roots, and, in some cases, these roots coalesce totally along the buccal surface (AT-101, AT-22, AT-21).

Lower second molar $\left(\mathrm{M}_{2}\right)$ With the exception of the mesial root of AT-3890 and the fractures in the distal two-thirds of the roots of AT142, AT-2273, AT-284, and AT-169, the preservation of the SH $\mathrm{M}_{2} \mathrm{~S}$ is excellent (Fig. 19, Table 21).

Compared to the $\mathrm{M}_{1} \mathrm{~s}$, the occlusal contour of the $\mathrm{SH} \mathrm{M}_{2} \mathrm{~s}$ is more squared than oval, and the limits of each cusp are less demarcated in the external contour of the tooth. In $28.6 \%$ of individuals, the hypoconulid is not expressed (e.g., individuals XI and XIX), and it only achieves a moderately large size (grade $\geq 3$ ) in $33.4 \%$ of individuals (e.g., AT-4147, AT-3889), never reaching the highest degree of the scale (grade 5). In addition, the position of the hypoconulid tends to be more central than it is in the $\mathrm{M}_{1}$ s. The central groove is significantly lingually offset, making the buccal cusps generally larger than the lingual cusps.

All of the $\mathrm{M}_{2}$ s have an anterior fovea, and, in $76.2 \%$ of cases, this is deep, with a tendency to be displaced buccally. Its conformation is more pit-like than lineal, and its expression is concomitant in all individuals, with a middle trigonid crest that is continuous in $90.5 \%$ of individuals. In contrast, the distal trigonid crest is absent in most of the specimens we examined. Only $23.8 \%$ of individuals express a weak crest joining the distal part of the protoconid and the metaconid, but this crest does not obliterate the central groove (e.g., AT-2193, individual XXVII).

The groove separating the metaconid from the entoconid is usually displaced mesially, significantly reducing the size of the metaconid. This circumstance is particularly pronounced when the $\mathbf{M}_{2}$ S express a C7. This accessory cusp is clearly absent in $8 \%$ of individuals, and, in individual $X X$, a more independent distal platform can be identified (grade 1). When the C7 is small (grade 2 in $30 \%$ ), it is expressed in the distal margin of the metaconid, delimited by a " $V$ " groove that opens lingually. When the size of this cusp ranges from moderate (grade 3 ) to significantly large (grade 4) $(25 \%)$, its position is central along the lingual border, its shape more rectangular, and it tends to invade the space of the metaconid and the entoconid (e.g., individual XI, AT-142, AT-1761). In AT-1761, the 


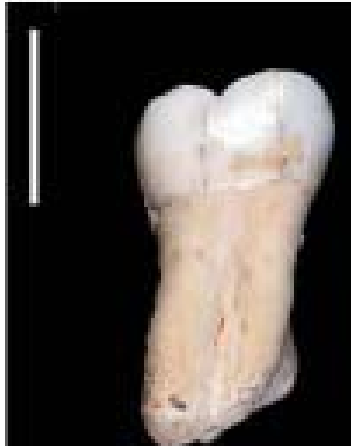

a

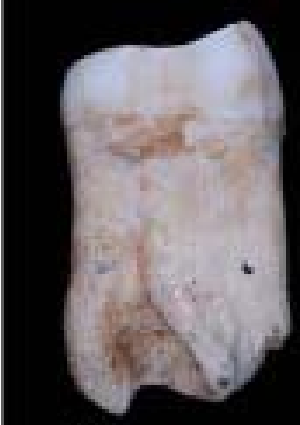

g

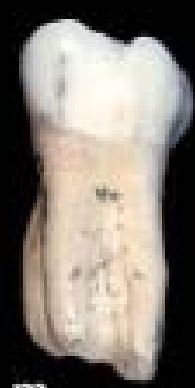

m

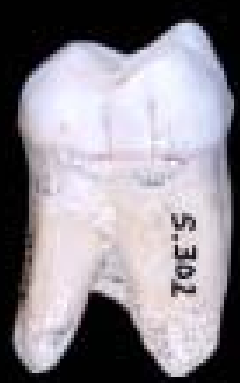

b

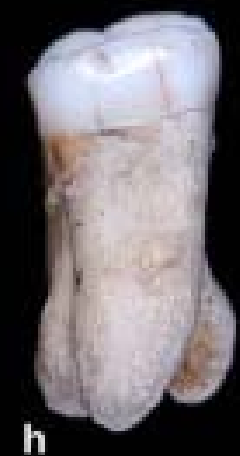

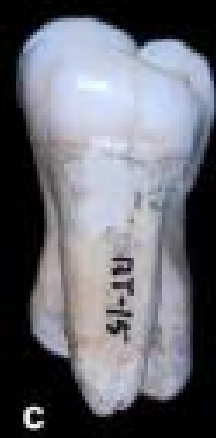

C
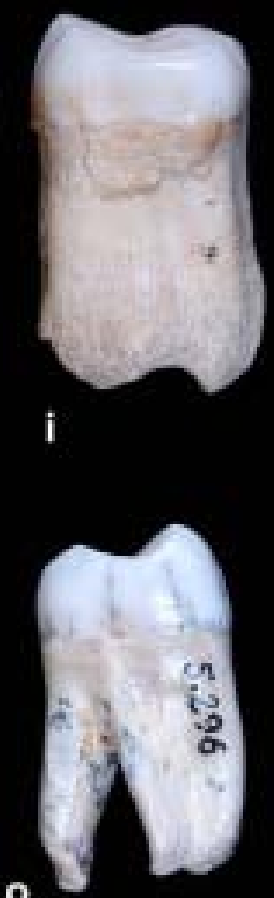

d

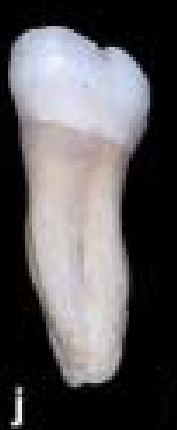

e

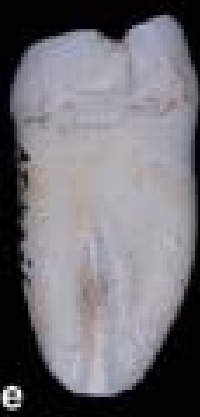

f
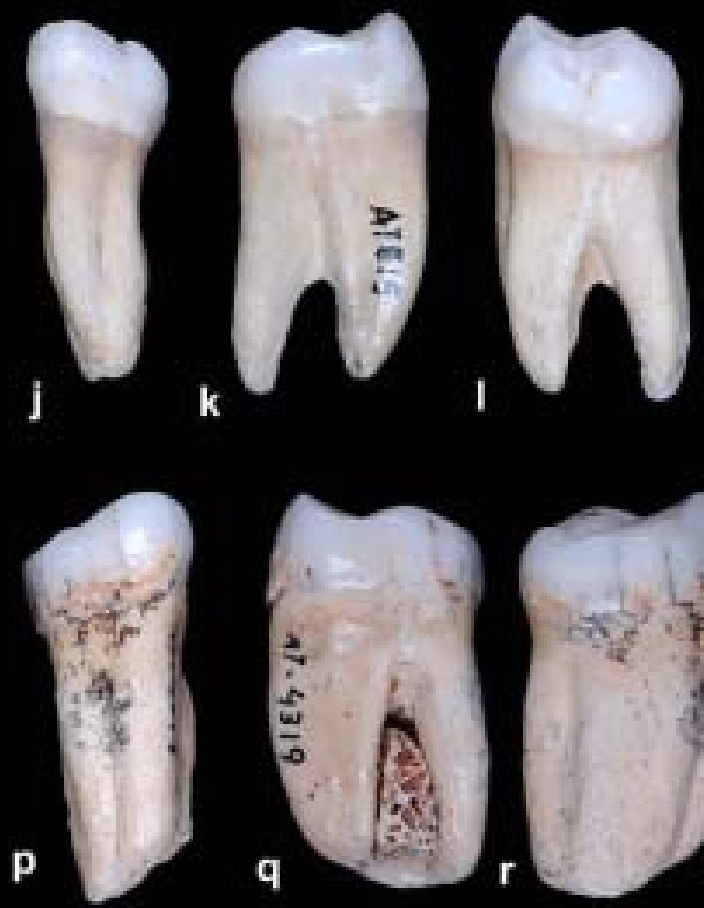
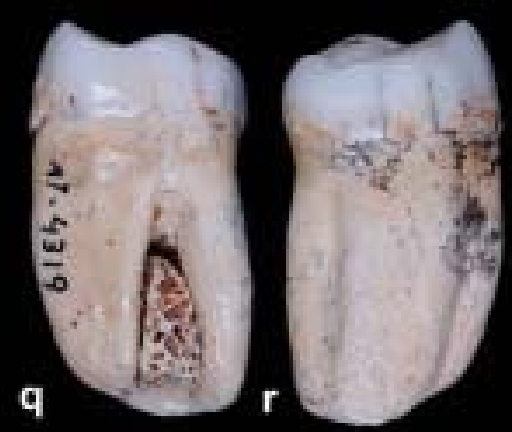

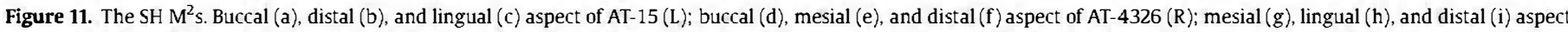

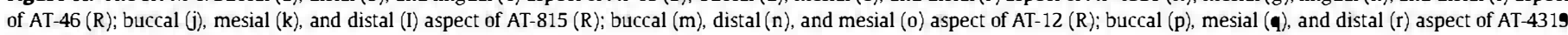
(L). L: left, R: right. White bar $=1 \mathrm{~cm}$.

entoconid is so reduced and displaced that it acquires the appearance of an accessory cusp.

The metaconid essential ridge is slightly deviated only in individuals I and XXII. There are no cases of a conspicuous deflecting wrinkle in the $\mathrm{M}_{2} \mathrm{SH}$ sample.

The C6 is present in this tooth type in $42.9 \%$ of the individuals. In $23.8 \%$ of cases its size is moderate (grade 2 ), and in $19.1 \%$ it equals the hypoconulid in size. Its position is always lingual, and its shape is triangular. There are some cases where the C6 is present despite the absence of a hypoconulid (see individuals I, XI, XXVI, and AT271 ), and in these cases, the C6 is placed between the hypoconid and the entoconid, but its position is lingual.

Compared to the $\mathrm{M}_{1} \mathrm{~s}$, the proportion of the $\mathrm{M}_{2} \mathrm{~s}$ with a Y-pattern is lower, being present in $40.9 \%$ of individuals. The predominant pattern is the "+" pattern, which is expressed in $36.3 \%$ of the individuals. There are two individuals (XVIII and XXII) in which one of the antimeres exhibits a "+" and the other an "X."

In $47.6 \%$ of individuals, the buccal groove separating the protoconid and the hypoconid on the buccal surface ends in a round pit that is classified as a protostylid in its lower degrees of expression (grade 1). This groove is generally smoother than in the $\mathbf{M}_{1}$ s. No cingulum is expressed.

All of the $\mathrm{M}_{2}$ s have two roots, one mesial and one distal, both of which are wide and mesiodistally compressed. The level of root bifurcation is generally low. We observe hypotaurodontism in $64.3 \%$ of specimens, and, in AT-2396, the pulp cavity extends to beyond the second third of the root length. From the approximal view, these roots have a pyramidal aspect, with the buccal margin being less curved than the lingual. Both the mesial and the distal roots have smooth longitudinal grooves delimiting two radicals each.

Third lower molar $\left(\mathrm{M}_{3}\right)$ With the exception of the distal two-thirds of the roots of AT-30, the $\mathrm{M}_{3} \mathrm{~s}$ of the SH sample exhibit an excellent state of preservation. Because the majority of the preserved specimens belonged to immature individuals, the root is not fully developed (Fig. 20, Table 22).

The contour of these teeth is an oval that is mesiodistally compressed. The morphology of the occlusal surface is highly 


\begin{tabular}{|c|c|c|c|c|c|c|}
\hline$M^{2}$ & Grade & SH & HEI & NEA & FSAP & SAP \\
\hline \multirow[t]{7}{*}{ Metacone size } & 0 & 0 & 0 & 0 & 0 & 0 \\
\hline & 1 & 0 & 0 & 0 & 0 & 0 \\
\hline & 2 & $3(16.7 \%)$ & 0 & $2(9.5 \%)$ & $1(8.3 \%)$ & $8(6.2 \%)$ \\
\hline & 3 & $8(44.4 \%)$ & $1(33.3 \%)$ & $9(42.9 \%)$ & $7(58.3 \%)$ & $45(346 \%)$ \\
\hline & 4 & $7(38.9 \%)$ & $2(66.7 \%)$ & $8(38.1 \%)$ & $3(25 \%)$ & $69(53.1 \%)$ \\
\hline & 5 & 0 & 0 & $2(9.5 \%)$ & $1(8.3 \%)$ & $8(6.2 \%)$ \\
\hline & Total & 18 & 3 & 21 & 12 & 130 \\
\hline \multirow[t]{7}{*}{ Hypocone size } & 0 & $3(16.7 \%)$ & 0 & $1(5.8 \%)$ & 0 & $9(6.9 \%)$ \\
\hline & $\mathbf{I}$ & $3(16.7 \%)$ & 0 & $1(4.8 \%)$ & $1(8.3 \%)$ & $9(6.9 \%)$ \\
\hline & 2 & $8(44.4 \%)$ & $1(33.3 \%)$ & $3(143 \%)$ & $5(41.7 \%)$ & $27(20.8 \%)$ \\
\hline & 3 & $1(5.6 \%)$ & $1(33.3 \%)$ & $6(28.6 \%)$ & $5(41.7 \%)$ & $31(23.8 \%)$ \\
\hline & 4 & $3(16.7 \%)$ & 0 & $9(42.9 \%)$ & $1(8.3 \%)$ & $42(32.3 \%)$ \\
\hline & 5 & 0 & $1(33.3 \%)$ & $1(4.8 \%)$ & 0 & $12(9.2 \%)$ \\
\hline & Total & 18 & 3 & 21 & 12 & 130 \\
\hline \multirow[t]{6}{*}{ C5 size } & 0 & $5(27.8 \%)$ & 0 & $2(10 \%)$ & $4(33.3 \%)$ & $71(55 \%)$ \\
\hline & 1 & $3(16.7 \%)$ & $1(50 \%)$ & $5(25 \%)$ & $2(16.7 \%)$ & $23(17.8 \%)$ \\
\hline & 2 & $4(22.2 \%)$ & 0 & $8(40 \%)$ & $3(25 \%)$ & $21(16.3 \%)$ \\
\hline & 3 & $3(16.7 \%)$ & 0 & $4(20 \%)$ & $2(16.7 \%)$ & $13(10.1 \%)$ \\
\hline & 4 & $3(16.7 \%)$ & $1(50 \%)$ & $1(5 \%)$ & $1(8.3 \%)$ & $1(0.8 \%)$ \\
\hline & Total & 18 & 18 & 20 & 12 & 129 \\
\hline \multirow[t]{3}{*}{ Crista obliqua } & 0 & $3(16.7 \%)$ & $1(33.3 \%)$ & $6(28.6 \%)$ & $5(41.7 \%)$ & $82(646 \%)$ \\
\hline & 1 & $15(83.3 \%)$ & $2(66 \%)$ & $15(71.4 \%)$ & $7(58.3 \%)$ & $45(35.4 \%)$ \\
\hline & Total & 18 & 3 & 21 & 12 & 127 \\
\hline \multirow[t]{3}{*}{ Transverse crest } & 0 & $13(76.5 \%)$ & $2(100 \%)$ & $13(76.5 \%)$ & $7(77.8 \%)$ & $48(42.5 \%)$ \\
\hline & 1 & $4(23.5 \%)$ & 0 & $4(23.5 \% 9$ & $2(22.2 \%)$ & $65(57.5 \%)$ \\
\hline & Total & 17 & 2 & 17 & 9 & 113 \\
\hline \multirow[t]{3}{*}{ Marginal accessory tubercles } & 0 & $5(38.5 \%)$ & $1(50 \%)$ & $3(18.8 \%)$ & $1(143 \%)$ & $51(49.5 \%)$ \\
\hline & 1 & $8(61.5 \%)$ & $1(50 \%)$ & $13(81.3 \%)$ & $6(85.7 \%)$ & $52(50.5 \%)$ \\
\hline & Total & 13 & 2 & 16 & 7 & 103 \\
\hline \multirow[t]{9}{*}{ Carabelli's complex } & 0 & $10(55.6 \%)$ & $1(50 \%)$ & $8(42.1 \%)$ & $7(70 \%)$ & $102(85.7 \%)$ \\
\hline & 1 & $6(33.3 \%)$ & 0 & $7(36.8 \%)$ & $2(20 \%)$ & $4(3.4 \%)$ \\
\hline & 2 & $2(11.1 \%)$ & 0 & $1(5.3 \%)$ & $1(10 \%)$ & $7(5.9 \%)$ \\
\hline & 3 & 0 & 0 & $2(10.5 \%)$ & 0 & $2(1.7 \%)$ \\
\hline & 4 & 0 & 0 & $1(5.3 \%)$ & 0 & $2(1.7 \%)$ \\
\hline & 5 & 0 & 0 & 0 & 0 & 0 \\
\hline & 6 & 0 & 0 & 0 & 0 & $2(1.7 \%)$ \\
\hline & 7 & 0 & $1(50 \%)$ & 0 & 0 & 0 \\
\hline & Total & 18 & 2 & 19 & 10 & 119 \\
\hline \multirow[t]{8}{*}{ Parastyle } & 0 & $18(100 \%)$ & $3(100 \%)$ & $19(100 \%)$ & $10(100 \%)$ & $116(97.5 \%)$ \\
\hline & $t$ & 0 & 0 & 0 & 0 & $2(1.7 \%)$ \\
\hline & 2 & 0 & 0 & 0 & 0 & 0 \\
\hline & 3 & 0 & 0 & 0 & 0 & 0 \\
\hline & 4 & 0 & 0 & 0 & 0 & 0 \\
\hline & 5 & 0 & 0 & 0 & 0 & $1(0.8 \%)$ \\
\hline & 6 & 0 & 0 & 0 & 0 & 0 \\
\hline & Total & 18 & 3 & 19 & 10 & 119 \\
\hline
\end{tabular}

variable, with a noticeable reduction of the size of the main cusps (particularly those of the talonid) and multiple secondary grooves and enamel crenulations. The cusps are not only reduced, but they occupy an unusual position within the occlusal contour and also exhibit atypical shapes, obscuring their identification.

With only one exception, all of the SH individuals express an anterior fovea in at least one of their antimeres, which is f requently (71.4\%) deep and pit-like (grade 2), and has a tendency to occupy a buccomesial position. From this fovea, secondary grooves may define the expression of accessory marginal tubercles with similar characteristics to the ones developed in the upper molars (see individual XVIII). This anterior fovea is associated with the expression of a thick middle trigonid crest (72.7\%), which joins the mesial portions of the protoconid and the metaconid and runs in an oblique mesiolingual to distobuccal direction (e.g., AT-2438, AT2760, AT-143).

Almost 70\% of the sample expresses a distal trigonid crest, and, in more than half of cases (56.6\%), it is conspicuous and continuous (grade 2). This crest represents the distal limit of the trigonid, which, in some cases, is exceptionally large in relation to the total occlusal surface (e.g., AT-2385, AT-2760, AT-2438).
The protoconid usually exhibits a clear cusp tip that tends to be displaced internally in relation to the external contour of the tooth. The metaconid rarely presents a clear essential ridge because almost its entire surface contributes to forming the middle and distal trigonid crest (see AT-2277). In some cases, the metaconid can only be distinguished by the expression of a short, deep groove separating both crests. This morphology obscures the identification of a deflecting wrinkle, such that there was only one case (AT-1959) where it was possible to identify certain deviation of the crest. The hypoconid, entoconid, and hypoconulid present a more or less triangular shape, and they are identified by their position within the occlusal surface. In $26.1 \%$ of individuals, at least one of the antimeres does not express a hypoconulid.

The C6 is frequently absent (63.6\%), and when it is present, it tends to be small (31.8\%). The C7 is far more frequent than the C6 (76.1\% present a grade $\geq 2$ ), though its size and morphology are highly variable within the SH population. In $42.8 \%$ of individuals, this accessory cusp is of a considerable size (grade $\geq 3$ ), tends to have a more trapezoidal than triangular shape, and fills the space of the adjacent cusps. In AT-942, the expression of the $C 7$ reduces the expression of the entoconid to a cuspal vestige. 


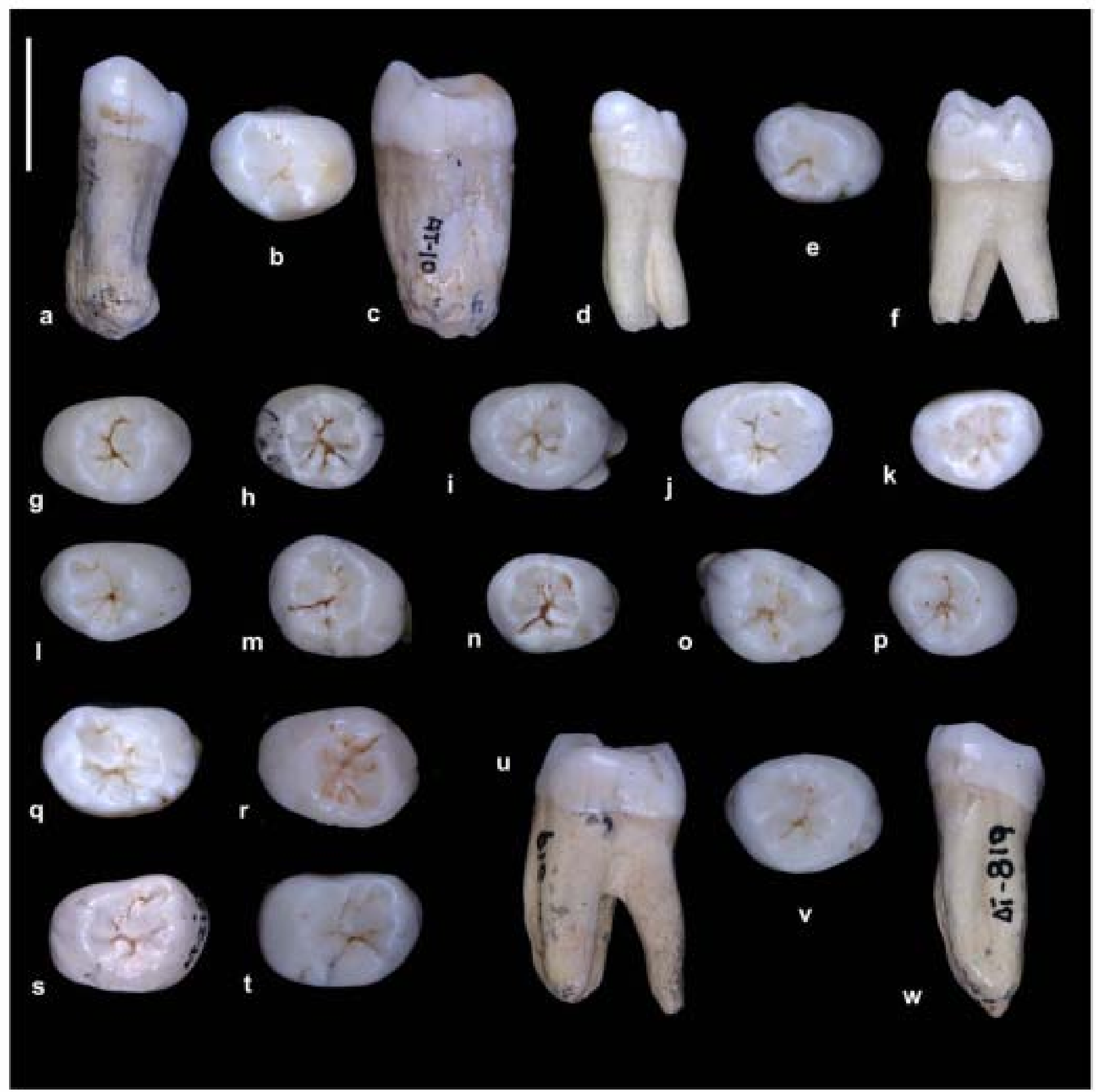

Figure 12. The SH M ${ }^{3}$ s. Buccal (a), occlusal (b), and distal (c) aspect of AT-10 (R); distal (d), occlusal (e), and distal (f) aspect of AT-816 (R). (L); i) AT-3181 (L); j) AT-171 (L); k) AT-826 (L); I) AT-601 (R); m) AT-945 (L); n) AT-1471 (L); o) AT-2393 (L); p) AT-3183 (L); q) AT-194 (R); r) AT-5082 (R); s) AT-2150 (L); t) AT- 140 (L); distal $(\mathrm{u})$, occlusal $(\mathrm{v})$, and buccal $(\mathrm{W})$ aspect of AT-819 $(\mathrm{R})$. Each occlusal image is positioned so its mesial aspect faces the top. L: left, $\mathrm{R}:$ right. White bar $=1 \mathrm{~cm}$.

In 27.3\% of individuals, at least one accessory cusp is evident in addition to the hypoconulid, and, in $31.8 \%$ of individuals, the expression of both the $\mathrm{C} 6$ and the $\mathrm{C} 7$ is concomitant with the hypoconulid. The size of the hypoconulid can range from small (grade 2 ) in $17.4 \%$ of the sample, to moderate (grade 3 ) and large (grades 4 and 5 ) in $26.1 \%$ and $21.7 \%$ of individuals, respectively.

Due to the intricate and crenulated occlusal pattern, it is difficult to assess the groove pattern of the $\mathrm{SH} \mathrm{M}_{3}$ s. In those individuals where it was possible to discern this, $23.5 \%$ of these cases present a dryopithecine conformation, whereas $41.2 \%$ exhibit an " $X$ " and $35.3 \%$ a ".$+ "$
Both mesial and distal views of this tooth type are similar, though the lingual surface is usually more vertical and the buccal surface is slightly inclined lingually. A protostylid of variable degrees of expression is present in $43.4 \%$ of individuals. However, in $23.8 \%$ of these cases, this is only in the form of a pit associated with the buccal groove (grade 1) or a short groove in the buccal aspect of the protoconid (grade 2 ). In $28.6 \%$ of the cases where it is present, its size ranges from moderate to large, even achieving the shape of a small cusp with a free apex in the case of AT-1473 (grade 6).

The root complex is formed by a mesial and a distal element, with frequent fusions and a tendency toward a delayed bifurcation. 


\begin{tabular}{|c|c|c|c|c|c|c|}
\hline $\mathrm{M}^{3}$ & Grade & SH & HEI & NEA & FSAP & SAP \\
\hline \multirow[t]{7}{*}{ Metacone size } & 0 & 0 & 0 & 0 & 0 & 0 \\
\hline & 1 & $2(9.5 \%)$ & 0 & $1(5.6 \%)$ & 0 & $2(2.2 \%)$ \\
\hline & 2 & $15(71.4 \%)$ & $1(50 \%)$ & $8(44.4 \%)$ & $5(50 \%)$ & $33(35.5 \%)$ \\
\hline & 3 & $4(19 \%)$ & $1(50 \%)$ & $6(33.3 \%)$ & $5(50 \%)$ & $42(45.2 \%)$ \\
\hline & 4 & 0 & 0 & $3(16.7 \%)$ & 0 & $16(17.2 \%)$ \\
\hline & 5 & 0 & 0 & 0 & 0 & 0 \\
\hline & Total & 21 & 2 & 18 & 10 & 93 \\
\hline \multirow[t]{7}{*}{ Hypocone size } & 0 & $12(57.1 \%)$ & $1(50 \%)$ & $3(17.6 \%)$ & $2(20 \%)$ & $15(16.1 \%)$ \\
\hline & 1 & $8(38.1 \%)$ & $1(50 \%)$ & $3(17.6 \%)$ & $1(10 \%)$ & $18(19.4 \%)$ \\
\hline & 2 & $1(4.8 \%)$ & 0 & $9(52.9 \%)$ & $7(70 \%)$ & $41(44.1 \%)$ \\
\hline & 3 & 0 & 0 & $2(11.8 \%)$ & 0 & $13(14 \%)$ \\
\hline & 4 & 0 & 0 & 0 & 0 & $6(6.5 \%)$ \\
\hline & 5 & 0 & 0 & 0 & 0 & 0 \\
\hline & Total & 21 & 2 & 17 & 10 & 93 \\
\hline \multirow[t]{6}{*}{ C5 size } & 0 & $20(95.2 \%)$ & $2(100 \%)$ & $6(35.3 \%)$ & $5(50 \%)$ & $63(67.7 \%)$ \\
\hline & 1 . & $1(4.8 \%)$ & 0 & $1(5.9 \%)$ & $1(10 \%)$ & $4(4.3 \%)$ \\
\hline & 2 & 0 & 0 & $3(17.6 \%)$ & $1(10 \%)$ & $8(8.6 \%)$ \\
\hline & 3 & 0 & 0 & $2(11.8 \%)$ & $1(10 \%)$ & $12(12.9 \%)$ \\
\hline & 4 & 0 & 0 & $5(29.4 \%)$ & $2(20 \%)$ & $6(6.5 \%)$ \\
\hline & Total & 21 & 2 & 17 & 10 & 93 \\
\hline \multirow[t]{3}{*}{ Crista obliqua } & 0 & $20(100 \%)$ & $2(100 \%)$ & $12(70.6 \%)$ & $8(80 \%)$ & $66(74.2 \%)$ \\
\hline & 1 & 0 & 0 & $5(29.4 \%)$ & $2(20 \%)$ & $23(25.8 \%)$ \\
\hline & Total & 20 & 2 & 17 & 10 & 89 \\
\hline \multirow[t]{3}{*}{ Transverse crest } & 0 & $19(95 \%)$ & $2(100 \%)$ & $13(92.9 \%)$ & $7(77.8 \%)$ & $65(77.4 \%)$ \\
\hline & 1 & $1(5 \%)$ & 0 & $1(7.1 \%)$ & $2(22.2 \%)$ & $19(22.6 \%)$ \\
\hline & Total & 20 & 2 & 14 & 9 & 84 \\
\hline \multirow[t]{3}{*}{ Marginal accessory tubercles } & 0 & $4(21.1 \%)$ & 0 & $2(143 \%)$ & $3(33.3 \%)$ & $29(35.8 \%)$ \\
\hline & 1 & $15(78.9 \%)$ & $2(100 \%)$ & $12(85.7 \%)$ & $6(66.7 \%)$ & $52(64.2 \%)$ \\
\hline & Total & 19 & 2 & 14 & 9 & 81 \\
\hline \multirow[t]{9}{*}{ Carabelli's complex } & 0 & $17(81 \%)$ & $2(100 \%)$ & $12(80 \%)$ & $5(55.6 \%)$ & $68(76.4 \%)$ \\
\hline & 1 & $1(4.8 \%)$ & 0 & 0 & $1(11.1 \%)$ & $6(6.7 \%)$ \\
\hline & 2 & 0 & 0 & $1(6.7 \%)$ & $2(22.2 \%)$ & $3(3.4 \%)$ \\
\hline & 3 & $1(4.8 \%)$ & 0 & $1(6.7 \%)$ & 0 & $1(1.1 \%)$ \\
\hline & 4 & $2(9.5 \%)$ & 0 & 0 & 0 & $3(3.4 \%)$ \\
\hline & 5 & 0 & 0 & 0 & $1(11.1 \%)$ & $3(3.4 \%)$ \\
\hline & 6 & 0 & 0 & 0 & 0 & $4(4.5 \%)$ \\
\hline & 7 & 0 & 0 & $1(6.7 \%)$ & 0 & $1(1.1 \%)$ \\
\hline & Total & 21 & 2 & 15 & 9 & 89 \\
\hline \multirow[t]{8}{*}{ Parastyle } & 0 & $20(95.2 \%)$ & $2(100 \%)$ & $14(87.5 \%)$ & $9(100 \%)$ & $81(92 \%)$ \\
\hline & 1 & 0 & 0 & $1(6.3 \%)$ & 0 & $3(3.4 \%)$ \\
\hline & 2 & 0 & 0 & $1(6.3 \%)$ & 0 & 0 \\
\hline & 3 & $1(4.8 \%)$ & 0 & 0 & 0 & 0 \\
\hline & 4 & 0 & 0 & 0 & 0 & $2(2.3 \%)$ \\
\hline & 5 & 0 & 0 & 0 & 0 & $1(1.1 \%)$ \\
\hline & 6 & 0 & 0 & 0 & 0 & $1(1.1 \%)$ \\
\hline & Total & 21 & 2 & 16 & 9 & 88 \\
\hline
\end{tabular}

The enlarged pulp cavity is particularly evident in specimens such as AT-605 or AT-13, where the bifurcation occurs beyond the second and the distal third of the roots, respectively.

\section{Comparative mor phology}

All pair-wise comparisons between groups showed significant MMD values except for $H$. heidelbergensis and the fossil $H$. sapiens sample (Table 23). Divergence between two samples was considered significant at the 0.25 level of probability when the MMD is greater than twice the standard deviation. However, we suggest reading this lack of significance between both groups with caution, because the SD can be affected by the small $\mathbf{N}$ for some of the dental traits in both the $\mathrm{H}$. heidelbergensis and the fossil $\mathrm{H}$. sapiens samples.

The greater similarities are held between $\mathrm{SH}$ and H. neanderthalensis (MMD $=0.137352$ ), followed by the contemporary and the fossil $H$. sapiens samples (MMD =0.142384). The most dissimilar groups are $H$. neanderthalensis and contemporary H. sapiens (MMD $=1.080199$ ), followed by SH and contemporary $H$. sapiens $(\mathrm{MMD}=1.008724)$. The similarities and dissimilarities are also illustrated in the MDS plot (Fig. 21), where SH hominins are closer (and thus, more similar) to $H$. neanderthalensis than to any other studied group, including the $H$. heidelbergensis taxon to which they are assigned. However, the MMD distance between SH and $H$. heidelbergensis is also small (MMD $=0.173322$ ), and smaller than that between $H$. heidelbergensis and $H$. neanderthalensis $(M M D=0.473684)$. The similarities identified between the non-SH $H$. heidelbergensis and the fossil $H$. sapiens are remarkable. Apart from potential limitations of the small $\mathbf{N}$ of these groups, the possible meaning of this association will be discussed below.

\section{Upper central incisors $\left(I^{1}\right)$}

The absolute and relative frequencies of labial convexity in the $I^{1} S$ are shown in Table 7 . The SH $I^{1} S$ invariably display the highest degrees and frequencies of labial convexity, similar to what is observed in $H$. neanderthalensis. The latter sample presents a wider range of variation due to its heterogeneity, which includes two specimens (one from Krapina and one from Saint-Césaire) with moderate or grade 3 labial curvatures. Unfortunately, the 


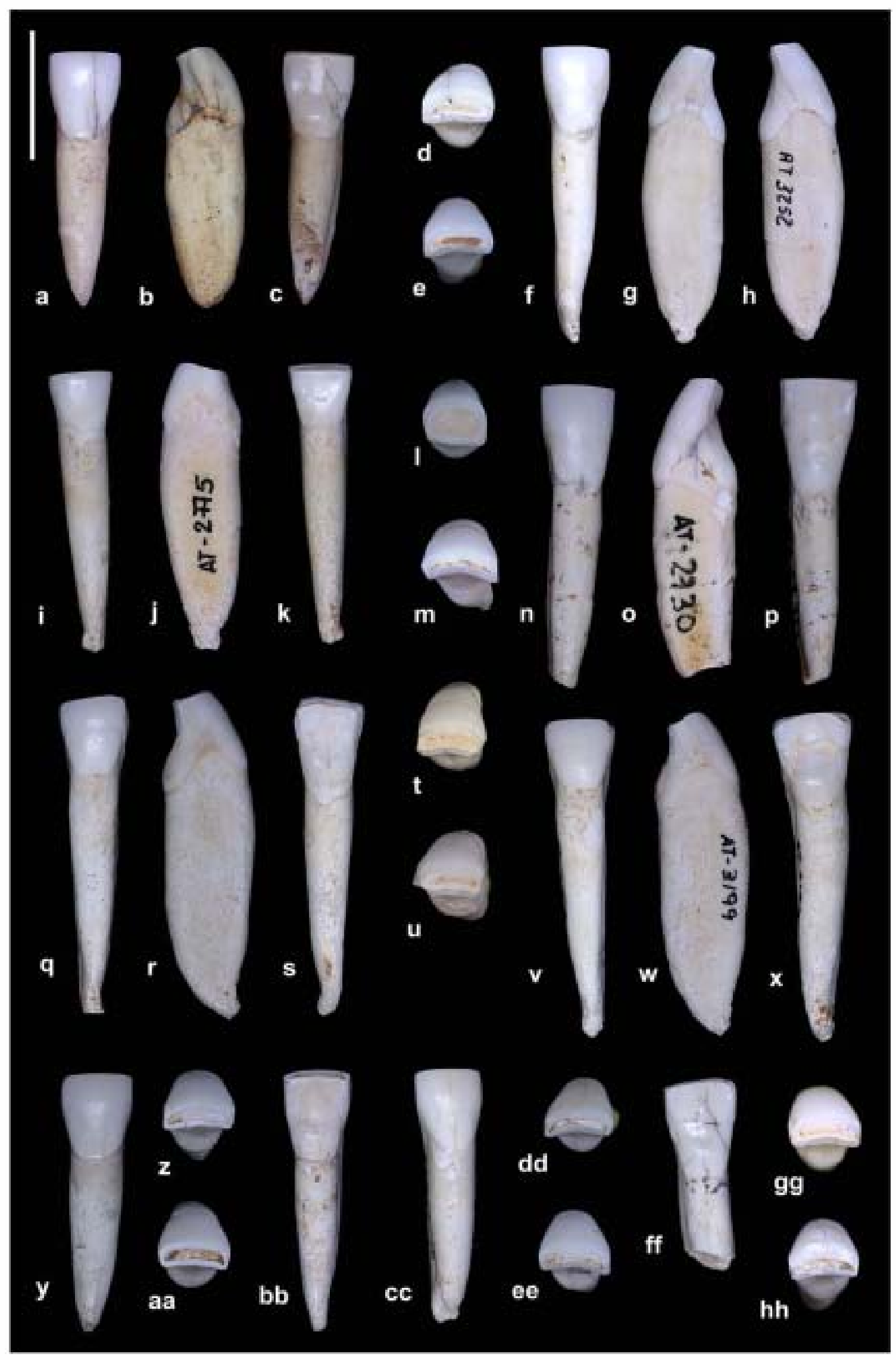

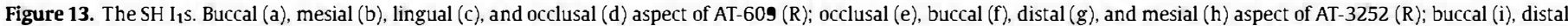

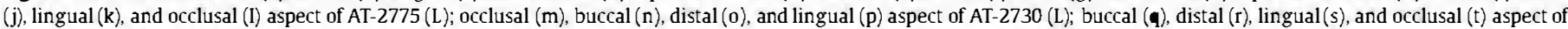

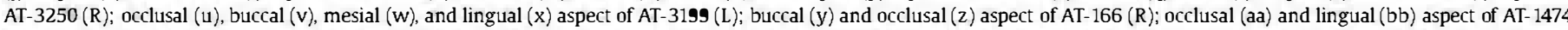

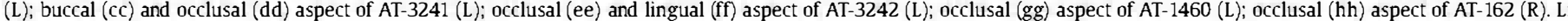
left, R: right. White bar $=1 \mathrm{~cm}$. 


\begin{tabular}{|c|c|c|c|c|c|c|}
\hline $\mathrm{I}_{1}$ & Grade & SH & HEI & NEA & FSAP & SAP \\
\hline \multirow[t]{7}{*}{ Labial convexity } & 0 & 0 & $1(25 \%)$ & 0 & $7(43.8 \%)$ & $58(59.8 \%)$ \\
\hline & 1 & 0 & $1(25 \%)$ & $6(66.7 \%)$ & $4(25 \%)$ & $26(26.8 \%)$ \\
\hline & 2 & $10(52.6 \%)$ & 0 & $3(33.3 \%)$ & $3(18.8 \%)$ & $11(11.3 \%)$ \\
\hline & 3 & $8(42.1 \%)$ & $2(50 \%)$ & 0 & $2(12.5 \%)$ & $2(2.1 \%)$ \\
\hline & 4 & $1(5.3 \%)$ & 0 & 0 & 0 & 0 \\
\hline & 5 & 0 & 0 & 0 & 0 & 0 \\
\hline & Total & 19 & 4 & 9 & 16 & 97 \\
\hline \multirow[t]{6}{*}{ Tuberculum dentale } & 0 & $14(73.7 \%)$ & $1(33.3 \%)$ & $5(50 \%)$ & $10(58.8 \%)$ & $87(89.7 \%)$ \\
\hline & 1 & $5(26.3 \%)$ & $2(66.7 \%)$ & $5(50 \%)$ & $5(29.4 \%)$ & $10(10.3 \%)$ \\
\hline & 2 & 0 & 0 & 0 & $1(5.9 \%)$ & 0 \\
\hline & 3 & 0 & 0 & 0 & $1(5.9 \%)$ & 0 \\
\hline & 4 & 0 & 0 & 0 & 0 & 0 \\
\hline & Total & 19 & 3 & 10 & 17 & 97 \\
\hline \multirow[t]{6}{*}{ Shovel shape } & 0 & $12(63.2 \%)$ & $2(50 \%)$ & $7(70 \%)$ & $10(62.5 \%)$ & $83(85.6 \%)$ \\
\hline & 1 & $5(26.3 \%)$ & $1(25 \%)$ & $2(20 \%)$ & $4(25 \%)$ & $12(12.4 \%)$ \\
\hline & 2 & $2(10.5 \%)$ & $1(25 \%)$ & $1(10 \%)$ & $2(12 \%)$ & $1(1 \%)$ \\
\hline & 3 & 0 & 0 & 0 & 0 & $1(1 \%)$ \\
\hline & 4 & 0 & 0 & 0 & 0 & 0 \\
\hline & Total & 19 & 4 & 10 & 16 & 97 \\
\hline
\end{tabular}

H. heidelbergensis sample is too worn to assess this trait, though Arago 111 may suggest a curvature that is at least moderate. In contrast, the fossil and contemporary $H$. sapiens specimens are mainly distributed along the lowest degrees of the scale for this trait, presenting the highest percentages of flat (grade 0 ) or almost flat labial (grade 1) surfaces (35.3\% and 69.3\%, respectively). However, fossil $H$. sapiens generally present more convex surfaces than the contemporary modern sample. In particular, the Qafzeh hominins range from grades 2 to 5 (Qafzeh 6 and 4), and the Dolní Věstonice individuals reach a maximum of grade 3 . With these exceptions, the highest degrees of labial convexity (grades 4 and 5) are exclusively presented by the Neanderthal and SH samples.

The SH hominins do not express strong ridging in the lingual surface but rather express an invariably round and well-developed basal eminence. Although we question whether or not the basal eminence and tuberculum dentale are homologous structures (for different opinions about its origin, see Scott and Turner [1997]; Bath-Balogh and Selma [1997]; and Martinón-Torres [2006]), we have recorded this basal eminence as a grade 1 (Table 7). This feature is also fairly common in the Neanderthal and $H$. heidelbergensis groups. However, in contrast to the SH sample, $H$. neanderthalensis, as a species, frequently expresses moderate to pronounced tubercles and ridges on the surface of this tooth type (e.g., Krapina). One of the specimens from the Arago sample shows clear lingual ridges (Arago 111), while another individual, Arago 61, displays a simple basal eminence that is similar to what is seen in $50 \%$ of the SH sample. H. sapiens, as a species, is, in general, characterized by low frequencies of $\mathbf{I}^{1}$ tuberculum dentale, with contemporary populations presenting higher proportions of almost or completely smooth lingual surfaces $(73.3 \%)$, vs. $31.3 \%$ of the fossil H. sapiens sample.

Shovel shape of this tooth type is a trait that characterizes H. neanderthalensis, having both high frequencies and degrees of expression (Table 7). Though this feature is also present in the majority of the SH hominins (90\%) to moderate degrees (grade $\leq 4$ ), it never reaches the highest grades on the expression scale (grades 5 and 6), overlapping with the $H$. heidelbergensis distribution for this trait. The fossil and contemporary $H$. sapiens samples also present differences for this trait, with the former presenting percentages of moderate to pronounced shoveling (64.7\%), similar to what is seen in $H$. heidelbergensis (75\%). Still, both of the $H$. sapiens samples that we investigated contain the highest proportion of non-shoveled incisors.
The MDS diagram (SOM Fig. 1) illustrates the general overlap of the SH and H. neanderthalensis samples. The only two SH specimens that fall out of the $H$. neanderthalensis 95\% confidence interval possess weak tuberculum dentale and shovel shape. One of the $H$. heidelbergensis individuals (Arago 111) falls outside of the $H$. neanderthalensis and Sima de los Huesos 95\% confidence intervals. The other $H$. heidelbergensis specimen (Arago 61), falls outside of the $H$. neanderthalensis variability but inside the $\mathrm{SH}$ range of variation. However, the inferences that can be made are limited by the missing values in some of the dental traits recorded for the Arago sample. Indeed, the small number of $H$. heidelbergensis $\mathrm{I}^{1} \mathrm{~s}$ that is available prevents a complete appreciation of their morphology, but even when they are worn, the Arago teeth present a massive aspect and apparently pronounced labial convexities. In general, the SH $\mathrm{I}^{1} \mathrm{~s}$ are more similar to the $H$. neanderthalensis $\mathrm{I}^{1} \mathrm{~S}$ than to any other group, particularly in considering the robust and massive morphology of this tooth type, its strong labial convexity, and the constant expression of a basal eminence. These also correspond with respect to the consistent development of a shovel shape, though $H$. neanderthalensis variability covers more pronounced forms, including that of the dental tubercle, than SH. This shovel shape is not associated with the development of a classic lingual fossa, which is typical of contemporary and early $H$. sapiens when this trait is expressed, because the basal eminence occupies the majority of the lingual surface.

\section{Upper lateral incisors $\left(\mathrm{I}^{2}\right)$}

The absolute and relative frequencies of the expression of labial convexity in all groups are shown in Table 8. Both the SH sample and the Neanderthals show highly pronounced labial curvatures (grade $\geq 4$ in $100 \%$ and $93.6 \%$, respectively), and the expression of a grade 5 labial curvature was only seen in these groups, with the exception of Qafzeh 5. Convexity is also pronounced in the two Arago teeth. The proportion of pronounced convexity is higher in the fossil $H$. sapiens sample (43\%) than in contemporary populations, which never exhibit higher than a grade 3 and display the highest percentage of flat surfaces. Indeed, grades 0 and 1 of this trait are exclusive to our contemporary $H$. sapiens sample.

The expression of the tuberculum dentale is highly variable within the SH population, ranging from complete absence (10.5\%) to the maximum degree of expression (5.3\%). Most of the population (58\%) presents a moderate to pronounced lingual tubercle 


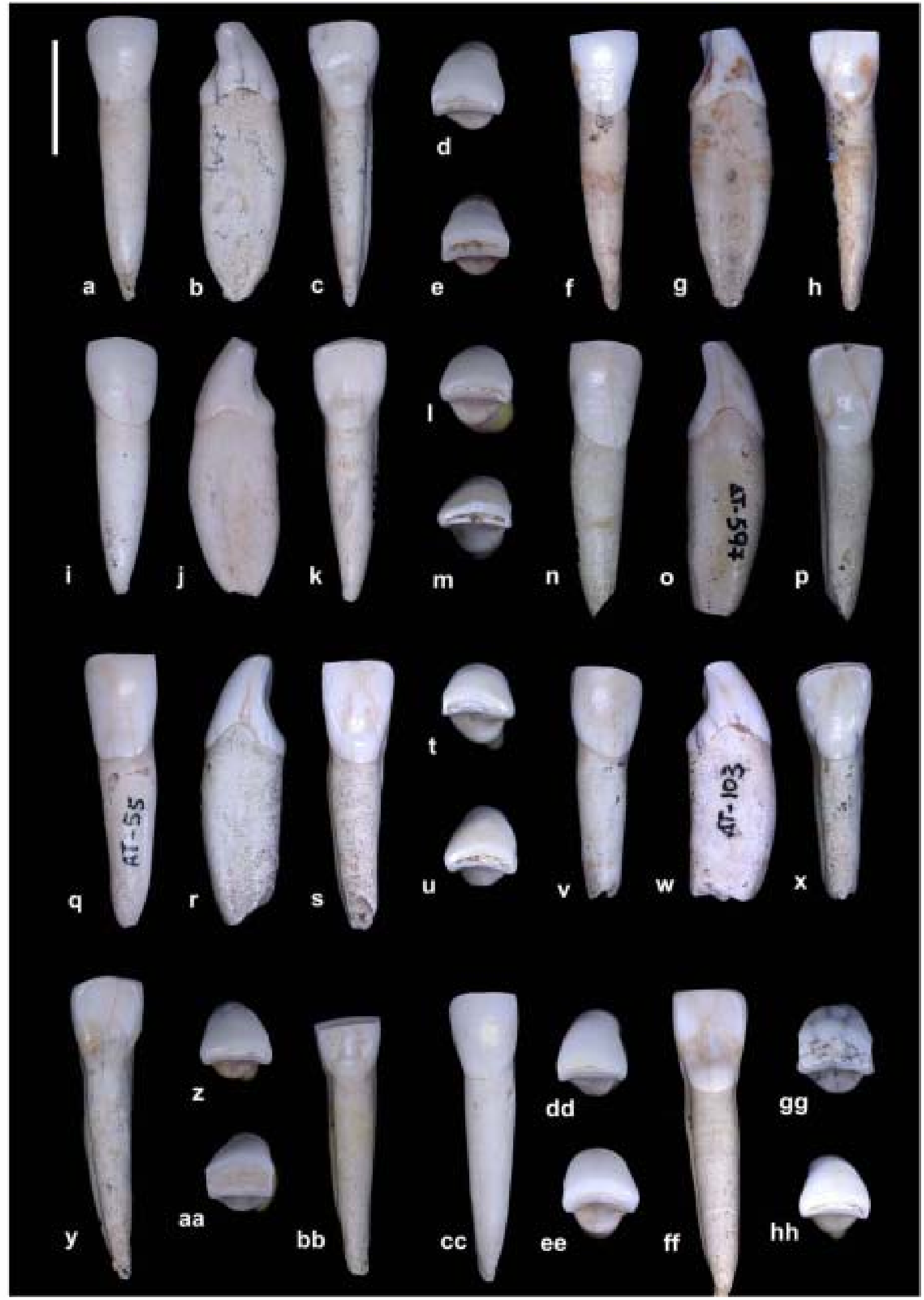

Figure 14. The SH I2s. Buccal (a), distal (b), lingual (c), and occlusal (d) aspect of AT-282 (R); occlusal (e), buccal (f), mesial ( $\mathrm{g}$ ), and lingual (h) aspect of AT-592 (R); buccal (i), distal (j), lingual (k), and occlusal (I) aspect of AT-2278 (L); occlusal (m), buccal (n), mesial (o), and lingual (p) aspect of AT-597 (L); buccal (q), mesial (r), lingual (s), and occlusal (t) aspect of AT-55 (R); $\operatorname{occlusal}(\mathrm{u})$, buccal $(\mathrm{v})$, mesial $(\mathrm{W})$, and lingual $(\mathrm{x})$ aspect of AT- $103(\mathrm{~L})$; lingual $(\mathrm{y})$ and occlusal $(\mathrm{z})$ aspect of AT-3937 (L); occlusal (aa) and lingual (bb) aspect of AT-2776 (L); buccal (cc) and occlusal (dd) aspect of AT-3256 (R); buccal (ee) and occlusal (ff) aspect of AT-1123 (R); occlusal aspects of (gg) AT-5 (R) and (hh) AT-167 (L). L: left, R: right. White bar $=1 \mathrm{~cm}$. 

contemporary H. supiens.

\begin{tabular}{|c|c|c|c|c|c|c|}
\hline $\mathrm{I}_{2}$ & Grade & SH & HEI & NEA & FSAP & SAP \\
\hline \multirow[t]{7}{*}{ Labial convexity } & 0 & 0 & 0 & 0 & $10(62.5 \%)$ & $82(60.3 \%)$ \\
\hline & 1 & 0 & 0 & $4(28.6 \%)$ & 0 & $38(27.9 \%)$ \\
\hline & 2 & $10(52.6 \%)$ & $1(50 \%)$ & $8(57.1 \%)$ & $4(25 \%)$ & $15(11 \%)$ \\
\hline & 3 & $9(47.4 \%)$ & 0 & $2(143 \%)$ & $2(12.5 \%)$ & $1(0.7 \%)$ \\
\hline & 4 & 0 & $1(50 \%)$ & 0 & 0 & 0 \\
\hline & 5 & 0 & 0 & 0 & 0 & 0 \\
\hline & Total & 19 & 2 & 14 & 16 & 136 \\
\hline \multirow[t]{6}{*}{ Tuberculum dentale } & 0 & $4(21.1 \%)$ & $1(50 \%)$ & $4(28.6 \%)$ & $7(43.8 \%)$ & $109(80.1 \%)$ \\
\hline & 1 & $14(73.7 \%)$ & $1(50 \%)$ & $9(643 \%)$ & $8(50 \%)$ & $26(19.1 \%)$ \\
\hline & 2 & $1(5.3 \%)$ & 0 & $1(7.1 \%)$ & $1(6.3 \%)$ & $1(0.8 \%)$ \\
\hline & 3 & 0 & 0 & 0 & 0 & 0 \\
\hline & 4 & 0 & 0 & 0 & 0 & 0 \\
\hline & Total & 19 & 2 & 14 & 16 & 136 \\
\hline \multirow[t]{6}{*}{ Shovel shape } & 0 & 0 & $1(50 \%)$ & $5(35.7 \%)$ & $8(50 \%)$ & $103(75.7 \%)$ \\
\hline & 1 & $7(36.8 \%)$ & 0 & $7(50 \%)$ & $8(50 \%)$ & $29(21.3 \%)$ \\
\hline & 2 & $8(42.1 \%)$ & $1(50 \%)$ & $2(142 \%)$ & 0 & $4(2.9 \%)$ \\
\hline & 3 & $4(21.1 \%)$ & 0 & 0 & 0 & 0 \\
\hline & 4 & 0 & 0 & 0 & 0 & 0 \\
\hline & Total & 19 & 2 & 14 & 16 & 136 \\
\hline
\end{tabular}

(grade $\geq 3$ ), but this percentage is higher in $H$. neanderthalensis (96.7\%), with $30 \%$ of the sample displaying a grade $\geq \mathbf{4}$ (e.g., Le Moustier and half of the Krapina population). The two Arago specimens do not display this cingular derivate. It is also important to note that, when shovel shape is pronounced, it may mask the identification of the tuberculum dentale, as in the SH specimens. Among the studied groups, fossil and contemporary $H$. sapiens samples present the highest proportion of absence or faint lingual tubercles (57.1\% and $70.2 \%$, respectively).

Both the $\mathrm{SH}$ and $H$. neanderthalensis groups present a pronounced $\mathrm{I}^{2}$ shovel shape with only three exceptions from the Krapina group, where only traces (grade 2) of it are expressed. In $H$. heidelbergensis, shoveling is also moderate. The contemporary $H$. sapiens group exhibits a wider range of expression, including a high number of individuals with faint or absent ridges (36.5\%), which is a morphological type that is exclusive to the contemporary $H$. sapiens sample. For the fossil $H$. sapiens sample, the proportion of moderate to pronounced thickening of the marginal ridges is higher than in the contemporary non-Asian sample, as is also the case for the expression of convexity and of the dental tubercle. However, apart from the comparison of shovel shape frequencies, it is important to note the morphological particularity of the Sima de los Huesos hominins and $H$. neanderthalensis in the development of this trait. In these two groups, we find a variant that we have called "triangular shovel shape" (Martinón-Torres et al., 2007a,b), where the marginal ridges are massive and highly conspicuous and invade the palatal face, defining a deep, longitudinal lingual fossa at their meeting point. The combination of this lingual morphology, together with a high degree of convexity of the labial surface, gives a characteristic "V-shape" morphology to the occlusal section. From the labial aspect, the marginal ridges run nearly parallel from the cervix to the incisal edge, forming a completely different shovel shape than the one assessed in $H$. sapiens (Mizoguchi, 1985; Crummett, 1995) and other hominin groups. In the Arago sample, we observe one specimen with "incipient triangular shape" (Martinón-Torres, 2006; Martinón-Torres et al., 2007b), which expresses a lingual longitudinal groove with a slightly wider fossa and divergent margins, and we observe one specimen exhibiting a "classic shovel shape" of grade 3. This incipient triangular shoveling has also been described in Homo erectus and Homo antecessor (Martinón-Torres et al., 2007b; Gómez-Robles, 2010). As an exception within the taxon $H$. sapiens, the specimens from Qafzeh have been observed to develop an incipient triangular shape where the marginal ridges are divergent, but with the convexity being pronounced and the shovel shape conspicuous. The MDS diagram (SOM Fig. 2) illustrates the general overlap of the $\mathrm{SH}$ and H. neanderthalensis distributions, with only three SH individuals falling out of the 95\% confidence interval of $H$. neanderthalensis, which is related to a less pronounced dental tuberculum expression in the former. Both $H$. heidelbergensis individuals fall outside of the two group distributions.

\section{Upper canines $\left(C^{\prime}\right)$}

The frequencies of the expression of tuberculum dentale in all groups are illustrated in Table 9. It is highly variable, although some general trends can be ascertained in some of the groups. Both the SH sample and $H$. neanderthalensis tend to show the highest frequencies of strong to pronounced (grades 3 and 4 ) lingual ridging (38.1\% and $52.4 \%$, respectively) and cuspule-like features (grades 5 and 6) (33.3\% and $47.6 \%$, respectively). However, the SH sample also presents some cases of weakly expressed tubercles (28.5\% are grades 1 and 2). Similarly, $H$. heidelbergensis covers a wide range of expression: from absence and faint ridging in the Arago specimens to a moderate tubercle in Mountmarin. In general, $H$. sapiens displays the highest percentage of individuals where this trait is absent or only slightly expressed. Within this species, the proportion of individualswith moderate to pronounced tubercles is higher in the fossil group than in the contemporary group (40\% vs. $9 \%$ ).

$H$. sapiens as a species displays low frequencies and degrees of expression of shovel shape, particularly in the contemporary populations (Table 9). In the remaining groups, the range of expression is wider, although the norm for the Middle Pleistocene hominins (SH and $H$. heidelbergensis) and $H$. neanderthalensis is to present a marked shovel shape, which never is lower than a grade 3 . The mesial ridge can be more developed than the distal one, a circumstance that occurs in the majority of the $\mathrm{SH}$ and $\mathrm{H}$. neanderthalensis samples (Table 9). In $78.9 \%$ of SH hominins and $42.1 \%$ of Neanderthals, the thicker mesial marginal ridge is attached to the dental tubercle, whereas in the contemporary and fossil $H$. sapiens samples this morphological type is relatively uncommon $(5.3 \%$ and $11.1 \%$, respectively).

The distal accessory ridge is the only trait that appears to show consistent sexual dimorphism (Scott and Turner, 1997) across human samples, so its value for taxonomic or phylogenetic purposes remains obscure when sex is unknown, as is the case for 


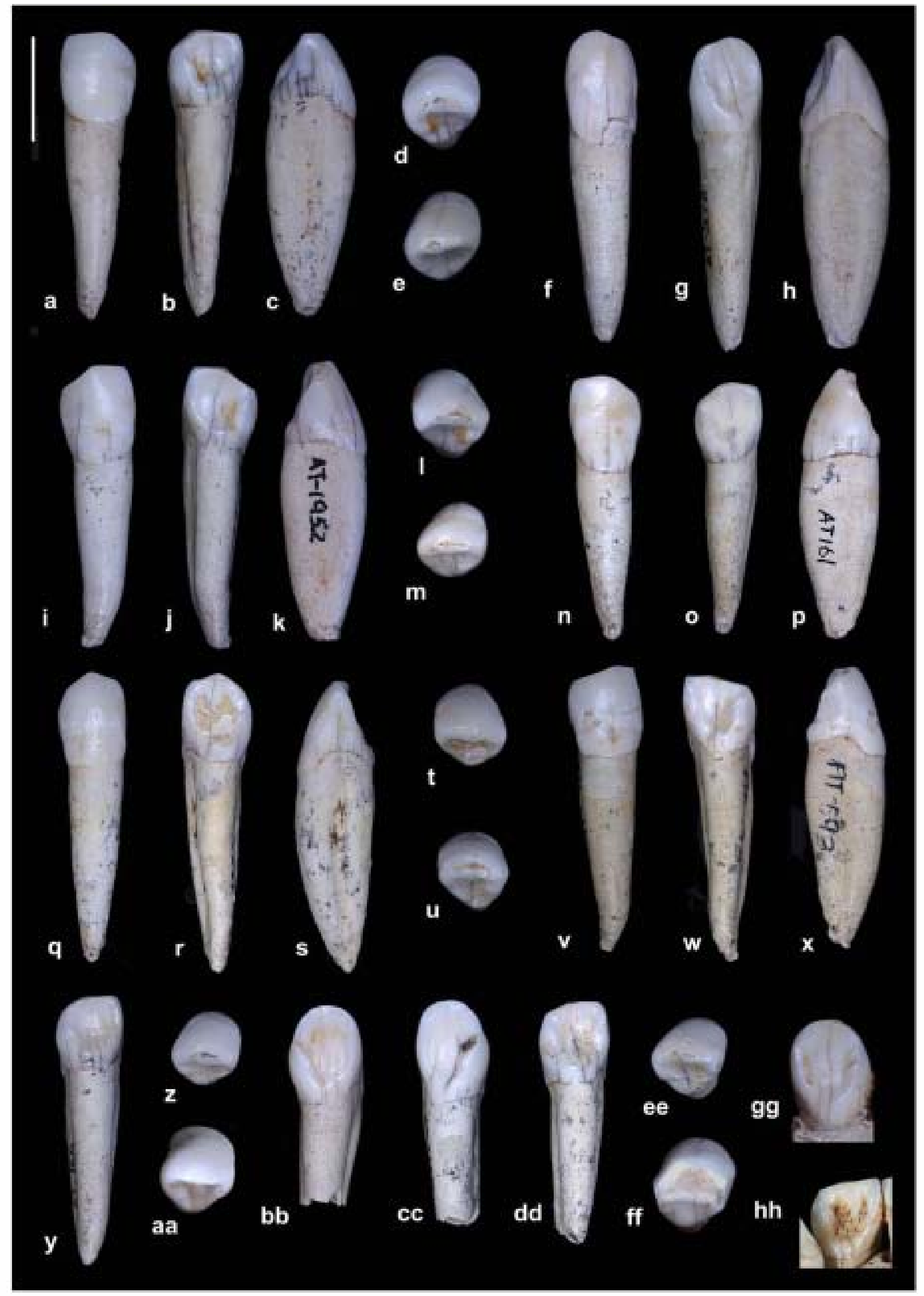

Figure 15. The SH C,s. Buccal (a), lingual (b), mesial (c), and occlusal (d) aspect of AT-276 (L); occlusal (e), buccal (f), lingual ( $\mathrm{g}$ ), distal (h), and occlusal (i) aspect of AT-578 (L); buccal $(\mathrm{j})$, distal $(\mathrm{k})$, lingual (I), and occlusal $(\mathrm{m})$ aspect of AT-1952 (R); occlusal (n), buccal (o), lingual (p), and distal (q) aspect of AT-161 (L); buccal (r), lingual (s), mesial (t), and occlusal (u) aspect of AT-3886 (R); $\operatorname{occlusal}(\mathrm{v})$, buccal (w), distal (x), and lingual (y) aspect of AT-593 (R); lingual (z) and occlusal (aa) aspect of AT-164 (L); occlusal (bb) aspect of AT-410 (L); lingual (cc) aspect of AT-2165 (R); occlusal (dd) and lingual (ee) aspect of AT-2438 (L); occlusal (ff) aspect of AT-145 (L); lingual aspects of (gg) AT-2 (R) and (hh) AT-607 (L). L: left, R: right. White bar $=1 \mathrm{~cm}$ 
Table 17

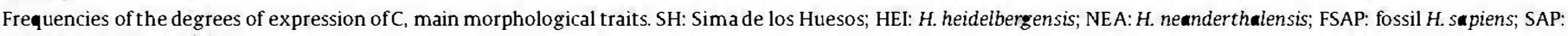
contemporary H. supiens.

\begin{tabular}{|c|c|c|c|c|c|c|}
\hline $\mathrm{C}$ & Grade & SH & HEI & NEA & FSAP & SAP \\
\hline \multirow[t]{6}{*}{ Tuberculum dentale } & 0 & $12(63.2 \%)$ & $5(100 \%)$ & $13(52 \%)$ & $7(41.2 \%)$ & $126(81.3 \%)$ \\
\hline & 1 & $7(36.8 \%)$ & 0 & $10(40 \%)$ & $8(47.1 \%)$ & $26(16.8 \%)$ \\
\hline & 2 & 0 & 0 & $2(8 \%)$ & $1(5.9 \%)$ & $2(1.3 \%)$ \\
\hline & 3 & 0 & 0 & 0 & $1(5.9 \%)$ & $1(0.6 \%)$ \\
\hline & 4 & 0 & 0 & 0 & 0 & 0 \\
\hline & Total & 19 & 5 & 25 & 17 & 155 \\
\hline \multirow[t]{7}{*}{ Shovel shape } & 0 & 0 & 0 & 0 & $2(11.8 \%)$ & $32(20.6 \%)$ \\
\hline & 1 & $1(5.3 \%)$ & 0 & $3(12 \%)$ & $4(23.5 \%)$ & $48(31 \%)$ \\
\hline & 2 & $3(15.8 \%)$ & $1(20 \%)$ & $7(28 \%)$ & $8(47.1 \%)$ & $55(35.5 \%)$ \\
\hline & 3 & $10(52.6 \%)$ & $2(40 \%)$ & $10(40 \%)$ & $1(5.9 \%)$ & $18(11.6 \%)$ \\
\hline & 4 & $4(21.1 \%)$ & $2(40 \%)$ & $5(20 \%)$ & $2(11.8 \%)$ & $2(1.3 \%)$ \\
\hline & 5 & $1(5.3 \%)$ & 0 & 0 & 0 & 0 \\
\hline & Total & 19 & 5 & 25 & 17 & 155 \\
\hline \multirow[t]{4}{*}{ Canine mesial ridge } & 0 & $19(100 \%)$ & $5(100 \%)$ & $20(80 \%)$ & $15(100 \%)$ & $32(97 \%)$ \\
\hline & 1 & 0 & 0 & $5(20 \%)$ & 0 & $1(3 \%)$ \\
\hline & 2 & 0 & 0 & 0 & 0 & 0 \\
\hline & Total & 19 & 5 & 25 & 15 & 33 \\
\hline \multirow[t]{4}{*}{ Distal accessory ridge } & 0 & $12(75 \%)$ & $2(50 \%)$ & $4(22.2 \%)$ & $7(58.3 \%)$ & $102(76.7 \%)$ \\
\hline & 1 & $4(25 \%)$ & $2(50 \%)$ & $5(27.8 \%)$ & $4(33.3 \%)$ & $23(17.3 \%)$ \\
\hline & 2 & 0 & 0 & $9(50 \%)$ & $1(8.3 \%)$ & $8(6 \%)$ \\
\hline & Total & 16 & 4 & 18 & 12 & 133 \\
\hline
\end{tabular}

most fossil material. This trait is present in about half of each population, with a higher proportion in the fossil $H$. sapiens sample (71.4\%). Based on the SH sex estimation done by Bermúdez de Castro et al. (2004a,b), it was possible to ascertain that all of the individuals assumed to be male present a distal accessory ridge of grade 2 , and that this trait was absent in those with undetermined sex. It was not possible to check its expression on individuals assumed to be female, due to severe wear.

Apart from these traits, we highlight a distinctive bulging of the upper half of the labial face in the C's of SH, Arago, and Mountmarin individuals, which could be a remnant of a cingulum. This trait is usually absent in $H$. neanderthalensis and $H$. sapiens. No buccal cingulum is expressed in any of the studied groups.

The MDS plot (SOM Fig. 3) illustrates the complete overlap of $\mathrm{SH}$ and $H$. heidelbergensis distributions with that of $H$. neanderthalensis.

\section{Upper third premolar $\left(P^{3}\right)$}

The morphology of the buccal essential crest is highly variable within the studied groups (see Table 10). A single crest is predominant in both the contemporary and the fossil $H$. sapiens groups, and it is also present in the Mountmarin specimen but not in Arago, where it is bifurcated. The bif urcated essential crest is the prevalent type in $H$. neanderthalensis (53.3\%), although the single crest is relatively frequent as well (40\%). Hortus IX is the only $H$. neanderthalensis in our sample presenting a flat buccal essential crest. However, this form is the predominant type in the SH sample (47.1\%), where the single and the bifurcated types are also present in similar proportions (29.4\% and $23.5 \%$, respectively).

Regarding the lingual essential crest, we also find high variability within groups (Table 10). As with the buccal crest, the single lingual essential crest is the most frequent type in both the early and contemporary $H$. sapiens groups. In the SH group, the single form is predominant (89.5\%), whereas grade 0 (the most frequent grade in the buccal cusp) is absent in its lingual counterpart. Bif urcated crests are the most frequent type in $H$. neanderthalensis $(66.7 \%)$ and $H$. heidelbergensis (100\%, $N=2$ ), and appear at variable frequencies in the remaining groups. The only case where the lingual essential crest is absent occurs in the contemporary H. sapiens.
The transverse crest is generally absent in all groups (Table 10), with only small percentages where an interrupted thin crest connects both main cusps (in one Krapina specimen and in five SH individuals). There is only one exceptional case (individual XX from $\mathrm{SH})$ where this crest is continuous.

The distal accessory ridge of the buccal cusp is present in one out of two $H$. heidelbergensis specimens and one out of two fossil $H$. sapiens specimens. Our contemporary $H$. sapiens sample presents the lowest frequency for this trait (10.5\%), followed by SH (28.6\%) and $H$. neanderthalensis (36.4\%). The mesial accessory ridge of the buccal cusp is even less frequent than its distal counterpart (Table 10). It is expressed in one $H$. heidelbergensis specimen (Arago 7), one Neanderthal from Krapina, and 14\% of the contemporary H. sapiens sample.

With regard to the expression of accessory marginal tubercles, SH and $H$. sapiens hominins tend to express only one tubercle (mostly in its mesial margin), while in $H$. neanderthalensis, the proportion of teeth with at least one mesial and one distal tubercle is slightly higher. When only one tubercle is expressed, in the majority of the cases it is distal.

Apart from the occlusal traits described above, we have ascertained a variable degree of bulging on the buccal surface of the $\mathrm{P}^{3}$, previously described by Weidenreich (1937) as a vestige of cingulum expression. This bulging is particularly pronounced in Arago 7 and some Neanderthals such as La Quina 5, Le Moustier, and Hortus IX, and also visible in the SH specimens (Bermúdez de Castro, 1987; Martinón-Torres, 2006; Gómez-Robles et al., 2011; this study).

By inspecting the MDS diagram (SOM Fig. 4), we can infer that the morphology of all of the $\mathrm{SH} \mathrm{P}^{3} \mathrm{~S}$ fall within the $H$. neanderthalensis range of variation. Both $H$. heidelbergensis specimens also fall within the $H$. neanderthalensis distribution although one of the $\mathrm{P}^{3} \mathrm{~s}$ (Mountmarin), is outside of the Sima de los Huesos 95\% confidence interval.

Although the root morphology was not analyzed in detail since many of the teeth were in situ, it is still possible to make some remarks. More than half of the $\mathrm{SH}^{3} \mathrm{~s}$ are double-rooted, a trait that it has been mentioned as characteristic of Neanderthals (de Lumley, 1973). Double-rooted $\mathrm{P}^{3} \mathrm{~s}$ are also common in the Arago sample, and, in the Hortus Neanderthal sample, single (e.g., Hortus VIII) and double-rooted (e.g., Hortus IX) specimens coexist. In our 


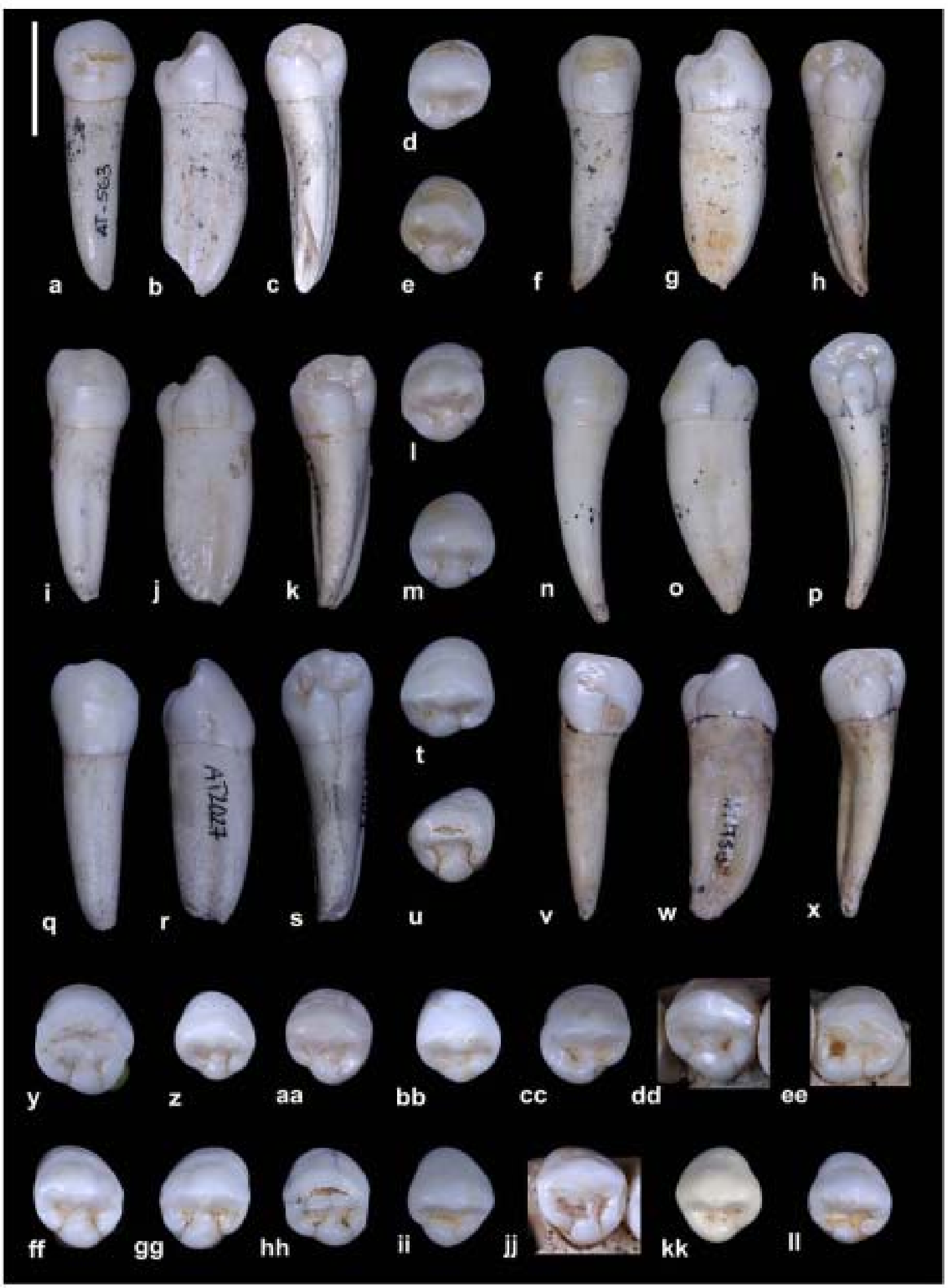

Figure 16. The SH P $\mathrm{P}_{3}$ s. Buccal (a), mesial (b), lingual (c), and occlusal (d) aspect of AT-563 (L); occlusal (e), buccal (f), distal ( $\mathrm{g}$ ), and lingual (h) aspect of AT-1466 (R); buccal (i), mesial (j), lingual (k), and occlusal (I) aspect of AT-1993 (L); occlusal (m), buccal (n), distal (o), and lingual (p) aspect of AT-4100 (L); buccal ( $(\mathrm{q})$, mesial (r), lingual (s), and occlusal ( $\mathrm{t}$ ) aspect of AT-2027 (R); occlusal (u), buccal (v), mesial (W), and lingual (x) aspect of AT-1760 (L); occlusal aspect of y) AT-590 (R); z) AT- 148 (R); aa) AT-3243 (R); bb) AT-3880 (R); cc) AT-2767 (L); dd) AT-300 (R); ee) AT-4147 (L); ff) AT-809 (R); gg) AT-3045 (L); hh) AT-2768 (R); ii) AT-2438 (R); ji) AT-607 (R); kk) AT-807 (L); and II) AT-3941 (R). L: left, R: right. White bar $=1 \mathrm{~cm}$ 

contemporary H. supiens.

\begin{tabular}{|c|c|c|c|c|c|c|}
\hline $\mathrm{P}_{3}$ & Grade & SH & HEI & NEA & FSAP & SAP \\
\hline \multirow[t]{7}{*}{ Lingual cusps } & 0 & 0 & 0 & 0 & $1(5.3 \%)$ & $75(50 \%)$ \\
\hline & 1 & $4(23.5 \%)$ & $2(20 \%)$ & $5(18.5 \%)$ & $7(36.8 \%)$ & $22(147 \%)$ \\
\hline & 2 & $9(52.9 \%)$ & $4(40 \%)$ & $7(25.9 \%)$ & $4(21.1 \%)$ & $34(22.7 \%)$ \\
\hline & 3 & $3(17.6 \%)$ & $3(30 \%)$ & $7(25.9 \%)$ & $4(21.1 \%)$ & $13(8.7 \%)$ \\
\hline & 4 & $1(5.9 \%)$ & $1(10 \%)$ & $8(29.6 \%)$ & $3(15.8 \%)$ & $6(4 \%)$ \\
\hline & 5 & 0 & 0 & 0 & 0 & 0 \\
\hline & Total & 17 & 10 & 27 & 19 & 150 \\
\hline \multirow[t]{4}{*}{ Transverse crest } & 0 & $3(16.7 \%)$ & $3(30 \%)$ & $4(15.4 \%)$ & $3(16.7 \%)$ & $105(66.9 \%)$ \\
\hline & 1 & 0 & $3(30 \%)$ & $8(30.8 \%)$ & $3(16.7 \%)$ & $32(20.4 \%)$ \\
\hline & 2 & $15(83.3 \%)$ & $4(40 \%)$ & $14(53.8 \%)$ & $12(66.7 \%)$ & $20(12.7 \%)$ \\
\hline & Total & 18 & 10 & 26 & 18 & 157 \\
\hline \multirow[t]{3}{*}{ Mesial accessory ridge } & 0 & $14(93.3 \%)$ & $7(77.8 \%)$ & $20(95.2 \%)$ & $11(91.7 \%)$ & $119(88.1 \%)$ \\
\hline & 1 & $1(6.7 \%)$ & $2(22.2 \%)$ & $1(4.8 \%)$ & $1(8.3 \%)$ & $16(11.9 \%)$ \\
\hline & Total & 15 & 9 & 21 & 12 & 13,521 \\
\hline \multirow[t]{3}{*}{ Distal accessory ridge } & 0 & $7(46.7 \%)$ & $6(66.7 \%)$ & $2(10 \%)$ & $8(53.3 \%)$ & $121(90.3 \%)$ \\
\hline & 1 & $8(53.3 \%)$ & $3(33.3 \%)$ & $18(90 \%)$ & $7(46.7 \%)$ & $13(9.7 \%)$ \\
\hline & Total & 15 & 9 & 20 & 15 & 134 \\
\hline
\end{tabular}

contemporary $H$. sapiens sample, the expression of both types is balanced ( $48.9 \%$ vs. $48.9 \%$ ), and, in $2.2 \%$ of cases, we find premolars with three roots by bifurcation of the buccal one.

\section{Upper fourth premolar $\left(P^{4}\right)$}

Regarding the buccal essential crest, a single ridge is the predominant type in the SH hominins and both fossil and contemporary $H$. sapiens (Table 11 ). $H$. heidelbergensis shows identical frequencies for single and bifurcated crests, and, in the $H$. neanderthalensis group, the bifurcated ridge is the prevailing form (66.6\% vs. 33.3\%). With only one exception in the contemporary $H$. sapiens group, flat buccal crests are absent in the studied sample.

The bifurcated lingual crest is the predominant form in $H$. neanderthalensis (Table 11) (e.g., Krapina, Arcy-sur-Cure, and Hortus) and $H$. heidelbergensis (e.g., Mountmarin and Arago vs. single crests in Steinheim and Pontnewydd). In the SH population, the proportion of single and bifurcated forms is balanced. In contrast, in the two $H$. sapiens samples, only nine specimens from the contemporary group (8.3\%) and none from the fossil $H$. sapiens sample present a bifurcated ridge.

In the majority of the studied samples, a transverse crest connecting both main cusps is absent (Table 11), and, when it exists, it is interrupted by the sagittal groove $(21.1 \%$ for $\mathrm{SH}, 6.2 \%$ in $H$. neanderthalensis, and $9.1 \%$ in fossil $H$. sapiens). There are only two exceptions, one from $H$. neanderthalensis (La Quina 5) and one from the early $H$. sapiens group (Q afzeh 6), where we can find a continuous enamel bridge connecting both cusps.

Approximately half of the $\mathrm{SH}$, the $\mathrm{H}$. neanderthalensis, and the fossil $H$. sapiens samples present a distal accessory ridge. This trait is present in all $H$. heidelbergensis specimens but Steinheim, and it is absent in the majority of the contemporary $H$. sapiens specimens (79.5\%). Compared to the distal accessory ridge, the expression of the mesial accessory ridge is lower, although half of the SH population and $H$. heidelbergensis sample express this trait.

The expression of accessory marginal tubercles is highly variable between and within groups. In all groups, there are specimens that present one or more accessory tubercles, and one $H$. heidelbergensis specimen and $19.5 \%$ of the contemporary $H$. sapiens sample express no accessory tubercles. The presence of two and even three accessory tubercles is slightly higher in SH than in Neanderthals. By inspecting the MDS diagram (SOM Fig. 5) we can infer that, regarding $\mathrm{P}^{4}$ morphology, all of the $\mathrm{SH}$ and the $H$. heidelbergensis specimens fall within the $H$. neanderthalensis range of variation.

Although an examination of root morphology was not an aim of this study, we can make a general assessment of its variation. The percentage of double-rooted $\mathrm{P}^{4} \mathrm{~S}$ in the $\mathrm{SH}$ sample (85.7\%) is slightly higher than in modern groups (Scott and Turner, 1997). In a previous study, we ascertained the presence of two roots in only $16 \%$ of the contemporary H. sapiens group (Martinón-Torres, 2006). The well-separated roots of the Sima de los Huesos $\mathrm{P}^{4} \mathrm{~S}$ are similar to those found in Mountmarin (6 B 3 and 14 B 3S) and La Chaise I 8/ 3 (Bermúdez de Castro, 1987).

In lateral view, the buccal surface presents a certain bulging that is less pronounced than in the $\mathrm{P}^{3}$. The cingulum, which is more typical of earlier Homo and Australopithecus groups, is absent in all the studied samples.

\section{Upper first molar $\left(M^{1}\right)$}

In general, all groups predominantly express a large metacone according to the ASUDAS scale (Table 12), with small and variable percentages of cases where this cusp is even very large (grade 5). There is only one exception, from the contemporary $H$. sapiens sample, where the metacone is expressed as a cuspule (grade 2).

The size of the hypocone is more variable among groups (Table 12). SH and $H$. neanderthalensis present the highest proportions of very large hypocones (grade $5 ; 64.7 \%$ and $52.2 \%$, respectively), with isolated single exceptions of small hypocones (grade 3 in individual XIX from SH, Arcy-sur-Cure, and Grotte du Renne 39). In $H$. heidelbergensis, the expression of the hypocone ranges from grades 4 to 5 . The majority of the $H$. sapiens specimens present a hypocone of large size, although, in the contemporary sample, the frequencies of grades 4 and 5 are very balanced (45.7\% and $43.3 \%$, respectively). Only in this species do we find a few cases where the hypocone is absent (grade 0) or weakly expressed as a cuspule (grade 2). However, and as we will discuss below, there are other morphological differences between the $H$. sapiens, $H$. heidelbergensis, and $H$. neanderthalensis groups that are not captured by the hypocone size assessment.

The expression of a C5 is highly variable both within and among the studied groups. It rarely adopts the shape of a cusp and never achieves the highest degree of development (grade 5), a circumstance that is fairly more frequent in early Homo and Australopithecus (Martinón-Torres, 2006). In a high number of cases, it has the aspect of an accessory ridge and sometimes its presence is only 


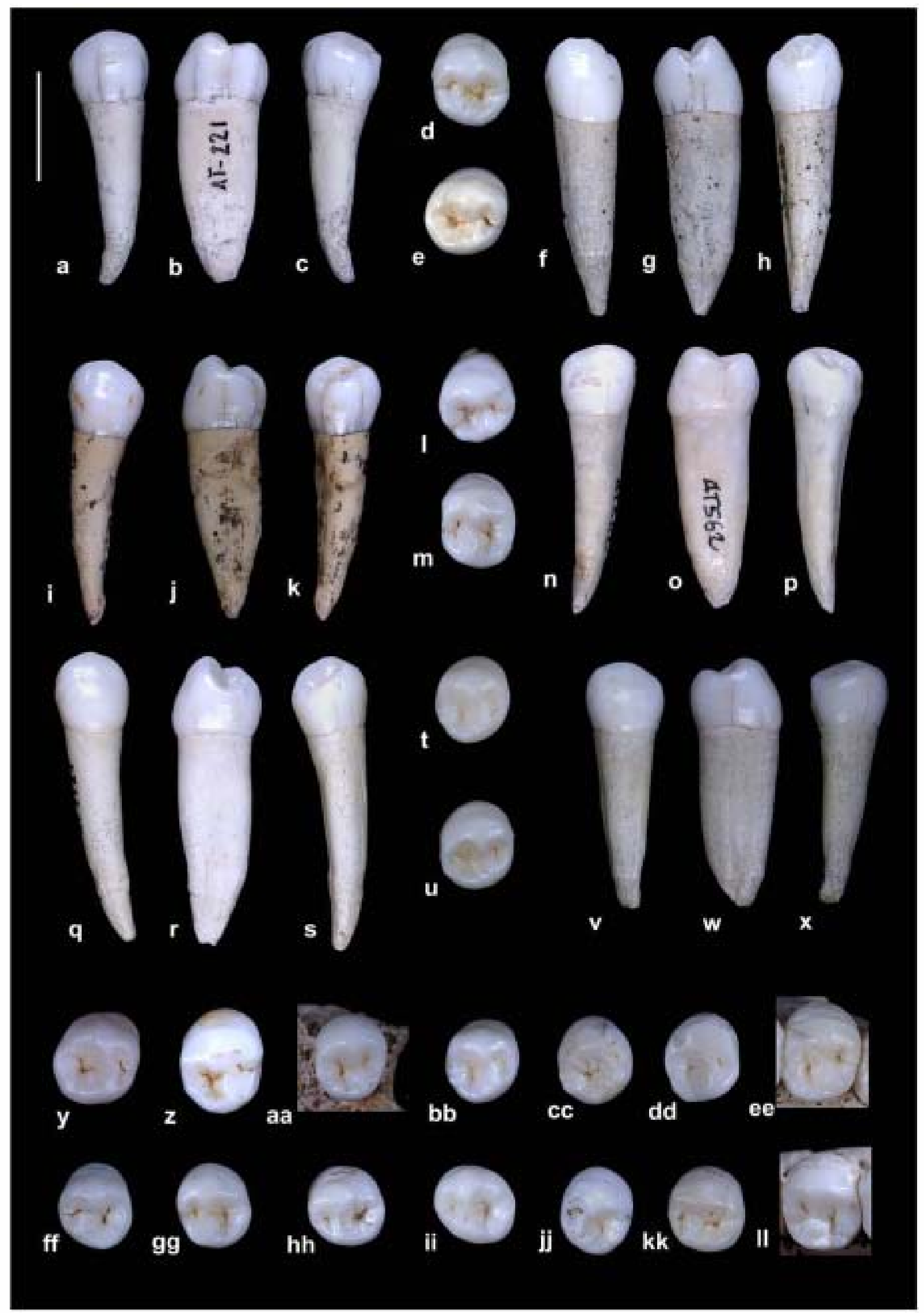

Figure 17. The SH P4s. Buccal (a), mesial (b), lingual (c), and occlusal (d) aspect of AT-221 (R); occlusal (e), buccal (f), mesial ( $\mathrm{g}$ ), and lingual (h) aspect of AT-603 (L); buccal (i), distal (j), lingual (k), and occlusal (I) aspect of AT-1763 (L); occlusal (m), buccal (n), mesial (o), and lingual (p) aspect of AT-562 (R); buccal ( $(\mathbf{q})$, distal (r), lingual ( $\mathrm{s})$, and occlusal (t) aspect of AT-2781 (L); occlusal (u), buccal (v), mesial (w), and lingual (x) aspect of AT-580 (L); occlusal aspect of y) AT-828 (L); z) AT- 147 (L); aa) AT-28 (L); bb) AT-2781 (L); cC) AT-2245 (L); dd) AT-4101 (L); ee) AT-4147 (L); ff) AT-3942 (R); gg) AT-28 (R); hh) AT-277 (R); ii) AT-168 (R); jj) AT-2780 (R); kk) AT-1467 (R); and II) AT-300 (R). L: left, R: right. White bar = 1 cm. 
Table 19

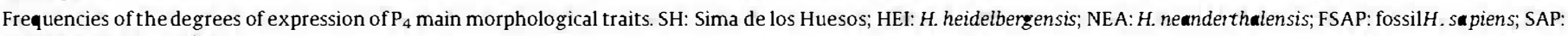
contemporary H. supiens.

\begin{tabular}{|c|c|c|c|c|c|c|}
\hline $\mathrm{P}_{4}$ & Grade & SH & HEI & NEA & FSAP & SAP \\
\hline \multirow[t]{7}{*}{ Lingual cusps } & 0 & 0 & 0 & 0 & $1(5 \%)$ & $20(14.1 \%)$ \\
\hline & 1 & 0 & 0 & 0 & $2(10 \%)$ & $20(141 \%)$ \\
\hline & 2 & 0 & 0 & 0 & $6(30 \%)$ & $69(48.6 \%)$ \\
\hline & 3 & $1(5 \%)$ & 0 & $3(11.1 \%)$ & $7(35 \%)$ & $14(9.9 \%)$ \\
\hline & 4 & $18(90 \%)$ & $3(60 \%)$ & $19(70.4 \%)$ & $4(20 \%)$ & $15(10.6 \%)$ \\
\hline & 5 & $1(5 \%)$ & $2(40 \%)$ & $5(18.5 \%)$ & 0 & $4(2.8 \%)$ \\
\hline & Total & 20 & 5 & 27 & 20 & 142 \\
\hline \multirow[t]{4}{*}{ Transverse crest } & 0 & $1(5 \%)$ & $1(20 \%)$ & $5(19.2 \%)$ & $11(45.8 \%)$ & $116(81.7 \%)$ \\
\hline & 1 & $4(20 \%)$ & $3(60 \%)$ & $3(11.5 \%)$ & $3(12.5 \%)$ & $14(9.9 \%)$ \\
\hline & 2 & $15(75 \%)$ & $1(20 \%)$ & $18(69.2 \%)$ & $10(41.7 \%)$ & $12(8.5 \%)$ \\
\hline & Total & 20 & 5 & 26 & 24 & 142 \\
\hline \multirow[t]{3}{*}{ Mesial accessory ridge } & 0 & $16(100 \%)$ & $5(100 \%)$ & $19(95 \%)$ & $9(64.3 \%)$ & $92(80 \%)$ \\
\hline & 1 & 0 & 0 & $1(5 \%)$ & $5(35.7 \%)$ & $23(20 \%)$ \\
\hline & Total & 16 & 5 & 20 & 14 & 115 \\
\hline \multirow[t]{3}{*}{ Distal accessory ridge } & 0 & $6(33.3 \%)$ & $1(20 \%)$ & $6(30 \%)$ & $5(33.3 \%)$ & $87(77.7 \%)$ \\
\hline & 1 & $12(66.7 \%)$ & $4(80 \%)$ & $14(70 \%)$ & $10(66.7 \%)$ & $25(22.3 \%)$ \\
\hline & Total & 18 & 5 & 20 & 15 & 112 \\
\hline
\end{tabular}

recognized by the " $\mathrm{V}$ " bif urcation of the distal groove. We highlight the high proportion of contemporary $H$. sapiens where this cusp is completely absent (35.2\%) compared to the rest of the groups; however, in the $\mathrm{SH}$ and $\mathrm{H}$. heidelbergensis samples, the proportion of $\mathrm{M}^{1} \mathrm{~s}$ without $\mathrm{C} 5$ is higher than in $H$. neanderthalensis and fossil H. sapiens.

The expression of a crista obliqua in $\mathbf{M}^{1}$ is the norm in later Homo species (Table 12). There are isolated exceptions in H. neanderthalensis (Arcy Renne 39), fossil H. sapiens (one from Le Rois and two from St Germain de la Rivière), and 7 out of 123 specimens (5.7\%) from the contemporary $H$. sapiens group where this crest is discontinuous. In contrast, the expression of a transverse crest connecting the mesial aspect of the protocone and the paracone is fairly more variable among groups (Table 12). However, this trait, together with the accessory marginal tubercles, is easily affected by wear, so their percentages should be interpreted with caution due to the high number of missing values. The percentage of specimens with or without transverse crests are relatively balanced in the SH and contemporary $H$. sapiens groups. Its presence is predominant in the $H$. heidelbergensis sample (except for Arago 31), and less frequent in early $H$. sapiens (30.8\%) and H. neanderthalensis (25\%). Accessory marginal tubercles are expressed in half of the $\mathrm{SH}$ and $H$. heidelbergensis samples; they are slightly more frequent in $H$. neanderthalensis (63.2\%) and early H. sapiens (58.3\%), and less frequent in the contemporary $H$. sapiens group (34.6\%) (Table 12).

Both Carabelli's complex and the parastyle are traits with a high inter-population variability (Scott and Turner, 1997; MartinónTorres, 2006), although it has been observed that its frequency tends to be higher in Australopithecus and early Homo species compared to more recent hominins (e.g., Hlusko, 2003; Irish and Guatelli-Steinberg, 2003) but not exclusive to these groups. In the particular sample studied here, only in exceptional cases can we determine the presence of a Carabelli's trait reaching the shape of a medium-sized or large cusp (grades 6 and 7) (e.g., in H. neanderthalensis [one specimen from Krapina and one from Arcy Renne] and 10 out of 124 specimens from the contemporary $H$. sapiens sample). In the remaining groups, it never exceeds grade 4, and, in $\mathrm{SH}$, it is absent or expressed as a groove in the lingual surface of the protocone and cannot always be certified as a Carabelli's complex. The parastyle is fairly more uncommon than the Carabelli's trait (Table 12), being absent or only dubiously present as a pit between the paracone and the metacone. There are only isolated cases where a small cuspule was identified in the buccal surface of the tooth (one from the contemporary $H$. sapiens group and two from the $H$. neanderthalensis group [one each from Krapina and Hortus]).

Although this study focuses on the morphology of the crowns of the permanent dentition, some remarks can be made regarding the root morphology. The relatively high percentages of taurodontism found in Sima de los Huesos falls within the range of expression of this trait for H. neanderthalensis (e.g., Trinkaus, 1983; Kricum et al., 1999; Kupczik and Hublin, 2010).

Moreover, it is important to highlight that, despite the clear $\mathbf{M}^{1}$ shape differences between $H$. sapiens and $H$. neanderthalensis (Martinón-Torres, 2006; Gómez-Robles et al., 2007; MartinónTorres et al., 2007a,b; Bailey et al., 2009; Quam et al., 2009; Gómez-Robles, 2010), the ASUDAS and modified ASUDAS employed in this study (Martinón-Torres et al., 2007a) do not capture this diagnostic morphology. These differences refer to the general shape of the $\mathrm{M}^{1} \mathrm{~s}$ and the spatial configuration of the cusps, traits that are ideally assessed with geometric morphometric methods. Our own assessment of this tooth class (Martinón-Torres, 2006; Gómez-Robles et al., 2007; Gómez-Robles, 2010) confirmed that $H$. neanderthalensis presents a unique morphology characterized by a rhomboidal contour, a relatively distal displacement of the lingual cusps, and protrusion in the external outline of a large and bulging hypocone (Bailey, 2004). However, this conformation is not exclusive to this group, but already present in the European Early and Middle Pleistocene such as the Gran Dolina-TD6, Steinheim, SH, and Pontnewydd groups (with a slightly less pronounced degree in some of them) (Gómez-Robles et al., 2007; Martinón-Torres et al., 2007a,b). Particularly, the Arago group presents a more primitive configuration (Gómez-Robles et al., 2007) in accordance with previous observations of this sample (Bermúdez de Castro et al., 2003a). In contrast, contemporary and early $\mathrm{H}$. sapiens retain the primitive shape, with an approximately squared occlusal polygon and a regular contour in which no cusp protrudes in the external outline.

The MDS plot for the $\mathrm{M}^{1}$ (SOM Fig. 6) shows that the SH and $H$. heidelbergensis distributions fall within the $H$. neanderthalensis range of variation.

\section{Upper second molar $\left(M^{2}\right)$}

With the ASUDAS scales, we do not ascertain great variations in metacone size among groups (Table 13). There is a general trend toward its reduction from $\mathbf{M}^{1}$ to $\mathbf{M}^{2}$, although its size commonly ranges from medium to large (grades 3 and 4 ). None of the 


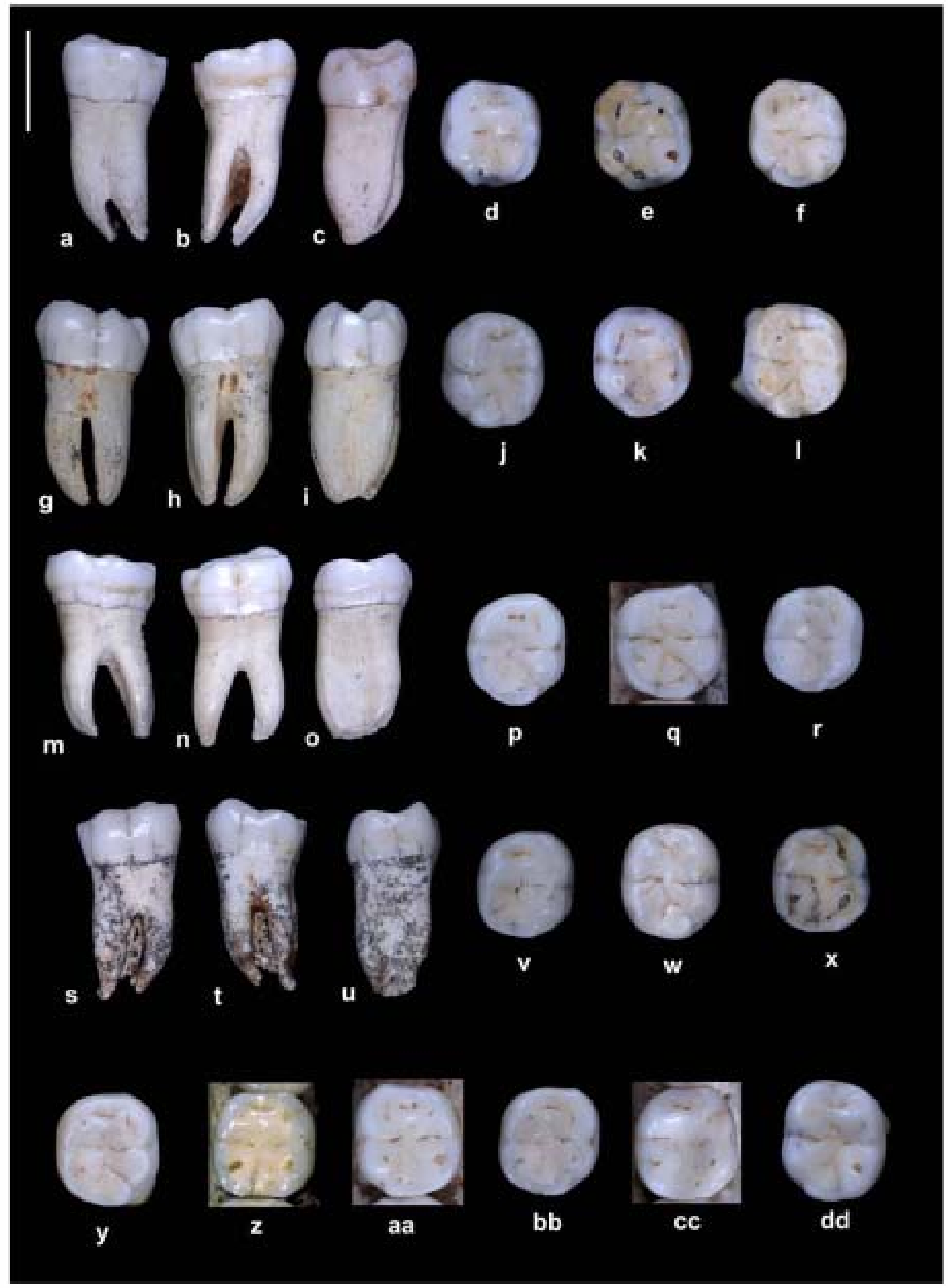

Figure 18. The SH M $\mathrm{M}_{1}$ s. Buccal (a), lingual (b), mesial (c), and occlusal (d) aspect of AT-22 (L); occlusal aspect of (e) AT-1759 (L) and (f) AT-286 (L); buccal ( $\mathrm{g}$ ), lingual (h), distal (i), and occlusal (j) aspect of AT-829 (L); occlusal aspect of $(\mathrm{k})$ AT- $556(\mathrm{~L})$ and $(\mathrm{I})$ AT- $1459(\mathrm{~L})$; buccal $(\mathrm{m})$, distal $(\mathrm{n})$, lingual $(\mathrm{o})$, and occlusal (p) aspect of AT-141 (R); occlusal aspect of $(\mathbf{q})$ AT$1(\mathrm{R})$ and $(\mathrm{r}) \mathrm{AT}-272(\mathrm{R})$; buccal $(\mathrm{s})$, lingual ( $\mathrm{t})$, distal $(\mathrm{u})$, and occlusal $(\mathrm{v})$ aspect of AT-2438 (R); occlusal aspect of $(\mathrm{w}) \mathrm{AT}-\mathrm{943}(\mathrm{R})$ and $(\mathrm{x}) \mathrm{AT}-561(\mathrm{R})$; occlusal aspect of $\mathrm{y}) \mathrm{AT}-2276(\mathrm{R})$; 2) AT-792 (R); aa) AT-1 (L); bb) AT-101 (R); cc) AT-300 (R); and dd) AT-4318 (L). L: left, R: right. White bar $=1 \mathrm{~cm}$. 
Table 20

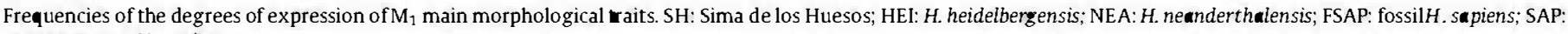
contemporary H. sapiens.

\begin{tabular}{|c|c|c|c|c|c|c|}
\hline $\mathrm{M}_{1}$ & Grade & $\mathrm{SH}$ & HEI & NEA & FSAP & SAP \\
\hline \multirow[t]{4}{*}{ Anterior fovea } & 0 & 0 & $1(14.3 \%)$ & $1(3.1 \%)$ & $11(61.1 \%)$ & $90(78.9 \%)$ \\
\hline & 1 & $3(15 \%)$ & $2(28.6 \%)$ & $5(15.6 \%)$ & $6(33.3 \%)$ & $22(19.3 \%)$ \\
\hline & 2 & $27(85 \%)$ & $4(57.1 \%)$ & $26(81.3 \%)$ & $1(5.6 \%)$ & $2(1.8 \%)$ \\
\hline & Total & 20 & 7 & 32 & 18 & 114 \\
\hline \multirow[t]{4}{*}{ Middle rrigonid crest } & 0 & $1(5 \%)$ & $2(28.6 \%)$ & $3(10 \%)$ & $19(95 \%)$ & $88(86.3 \%)$ \\
\hline & 1 & 0 & $1(143 \%)$ & $11(36.7 \%)$ & 0 & $13(12.7 \%)$ \\
\hline & 2 & $19(95 \%)$ & $4(57.1 \%)$ & $16(53.3 \%)$ & $1(5 \%)$ & $1(1 \%)$ \\
\hline & Total & 20 & 7 & 30 & 20 & 102 \\
\hline \multirow[t]{4}{*}{ Distal trigonid crest } & 0 & $14(70 \%)$ & $3(50 \%)$ & $16(57.1 \%)$ & $13(65 \%)$ & $89(87.3 \%)$ \\
\hline & 1 & $1(5 \%)$ & $3(50 \%)$ & $8(28.5 \%)$ & $5(25 \%)$ & $9(8.8 \%)$ \\
\hline & 2 & $5(25 \%)$ & 0 & $4(143 \%)$ & $2(10 \%)$ & $4(3.9 \%)$ \\
\hline & Total & 20 & 6 & 28 & 20 & 102 \\
\hline \multirow[t]{4}{*}{ Deflecting wrinkle } & 0 & $13(68.4 \%)$ & $3(50 \%)$ & $24(82.8 \%)$ & $16(84.2 \%)$ & $72(75.8 \%)$ \\
\hline & 1 & $4(21.1 \%)$ & $2(33.3 \%)$ & $4(13.8 \%)$ & $2(10.5 \%)$ & $5(5.3 \%)$ \\
\hline & 2 & $2(10.5 \%)$ & $1(16.7 \%)$ & $1(3.4 \%)$ & $1(5.3 \%)$ & $18(18.9 \%)$ \\
\hline & Total & 19 & 6 & 29 & 19 & 95 \\
\hline \multirow[t]{7}{*}{ Hypoconulid size } & 0 & $2(9.5 \%)$ & 0 & 0 & $1(3.6 \%)$ & $6(5 \%)$ \\
\hline & 1 & 0 & 0 & 0 & 0 & $1(0.8 \%)$ \\
\hline & 2 & 0 & 0 & $3(8.8 \%)$ & $2(7.1 \%)$ & $10(8.4 \%)$ \\
\hline & 3 & $2(9.5 \%)$ & 0 & $4(11.8 \%)$ & $7(25 \%)$ & $35(29.4 \%)$ \\
\hline & 4 & 9 (42.9\%) & $6(85.7 \%)$ & $16(47.1 \%)$ & $13(46.4 \%)$ & $52(43.7 \%)$ \\
\hline & 5 & $8(38.1 \%)$ & $1(143 \%)$ & $11(32.4 \%)$ & $5(17.9 \%)$ & $15(12.6 \%)$ \\
\hline & Total & 21 & 7 & 34 & 28 & 119 \\
\hline \multirow[t]{5}{*}{ C6 size } & 0 & $20(100 \%)$ & $4(66.7 \%)$ & $15(51.7 \%)$ & $17(65.4 \%)$ & $113(96.6 \%)$ \\
\hline & $t$ & 0 & $1(16.7 \%)$ & $4(13.8 \%)$ & $3(11.5 \%)$ & $1(0.9 \%)$ \\
\hline & 2 & 0 & 0 & $4(13.8 \%)$ & $4(15.4 \%)$ & $3(2.6 \%)$ \\
\hline & 3 & 0 & $1(16.7 \%)$ & $6(20.7 \%)$ & $2(7.7 \%)$ & 0 \\
\hline & Total & 20 & 6 & 29 & 26 & 117 \\
\hline \multirow[t]{6}{*}{ C7 size } & 0 & $10(50 \%)$ & $2(33.3 \%)$ & $9(31 \%)$ & $15(60 \%)$ & $96(82.8 \%)$ \\
\hline & 1 & $3(15 \%)$ & $2(33.3 \%)$ & $4(13.8 \%)$ & $5(20 \%)$ & $7(6 \%)$ \\
\hline & 2 & $3(15 \%)$ & $1(16.7 \%)$ & $8(27.6 \%)$ & $1(4 \%)$ & $5(43 \%)$ \\
\hline & 3 & $3(15 \%)$ & 0 & $6(20.7 \%)$ & $3(12 \%)$ & $6(5.2 \%)$ \\
\hline & 4 & $1(5 \%)$ & $1(16.7 \%)$ & $2(6.9 \%)$ & $1(4 \%)$ & $2(1.7 \%)$ \\
\hline & Total & 20 & 6 & 29 & 25 & 116 \\
\hline \multirow[t]{3}{*}{ Groove pattern } & 1 & $10(55.6 \%)$ & $4(57.1 \%)$ & $23(82.1 \%)$ & $17(77.3 \%)$ & $63(72.4 \%)$ \\
\hline & 2 & $8(44.4 \%)$ & $3(42.9 \%)$ & $5(17.9 \%)$ & $5(22.7 \%)$ & $24(27.6 \%)$ \\
\hline & Total & 18 & 7 & 28 & 22 & 87 \\
\hline \multirow[t]{9}{*}{ Protostylid } & 0 & $14(66.7 \%)$ & $6(100 \%)$ & $30(96.8 \%)$ & $23(88.5 \%)$ & $107(90.7 \%)$ \\
\hline & $\mathbf{T}$ & $7(33.3 \%)$ & 0 & $1(3.2 \%)$ & $3(11.5 \%)$ & $11(9.3 \%)$ \\
\hline & 2 & 0 & 0 & 0 & 0 & 0 \\
\hline & 3 & 0 & 0 & 0 & 0 & 0 \\
\hline & 4 & 0 & 0 & 0 & 0 & 0 \\
\hline & 5 & 0 & 0 & 0 & 0 & 0 \\
\hline & 6 & 0 & 0 & 0 & 0 & 0 \\
\hline & 7 & 0 & 0 & 0 & 0 & 0 \\
\hline & Total & 21 & 6 & 31 & 26 & 118 \\
\hline
\end{tabular}

$H$. heidelbergensis specimens present a metacone below grade 2 , and, in $\mathrm{SH}$, the percentage of this grade is $16.7 \%$.

There is a noticeable reduction of the size of the hypocone compared to the $\mathbf{M}^{1} \mathrm{~s}$ (Tables 12 and 13), achieving grades 0 and 1 in some specimens from the $\mathrm{SH}, H$. neanderthalensis, and both $H$. sapiens samples. $\mathrm{SH}$ is the group displaying the highest percentages of hypocone reduction.

The C5 is generally small, it occupies a central location along the distal margin, and its presence is generally ascertained by the distal groove bif urcation (without attaining volume most of the time). It is present in less than the half of the contemporary $\mathrm{H}$. sapiens specimens (45\%), in $66.6 \%$ of the fossil H. sapiens group, and in $72.2 \%$ of the SH population.

The reduction of the hypocone affects the occlusal outline of the $\mathbf{M}^{2}$, which tends to adopt a triangular or heart shape with the vertex oriented lingually, rather than the squared or rhomboidal contour typical of $\mathrm{M}^{1} \mathrm{~s}$. Within the H. heidelbergensis sample, Arago 14 displays a more primitive appearance with a rectangular outline, a large $\mathrm{C5}$, and an intricate pattern of crenulations and secondary fissures, in contrast to more derived specimens such as Pontnewydd and Steinheim.
The expression of a crista obliqua in the $\mathrm{M}^{2} \mathrm{~s}$ is more variable than in the $\mathbf{M}^{1} \mathrm{~s}$, but it is also affected by the more variable expression of the hypocone (Table 13). Still, the majority of the specimens (with the exception of the contemporary H. sapiens group) present this trait, as it is typical of Homo (Martinón-Torres, 2006). The transverse crest connecting the mesial aspect of the protocone and the paracone is less frequent but also easily affected by wear, so its percentages should be read with caution since there were many specimens we could not score. The contemporary $H$. sapiens group presents the highest frequencies for this trait $(57.5 \%)$; in the remaining groups, the crest is predominantly absent (Table 13). With regard to the accessory marginal tubercles, their presence-absence is relatively balanced in contemporary $\mathrm{H}$. sapiens (49.5\% vs. 50.5\%) and H. heidelbergensis (absent in Steinheim and present in Arago 14), while in the remaining groups, these tubercles are predominantly expressed. The frequencies are particularly high in $H$. neanderthalensis and early $H$. sapiens (Table 13 ).

Regarding the Carabelli's complex and the parastyle, only Arago 14 and two specimens from the contemporary $H$. sapiens group express a Carabelli's cusp with a free apex (Table 13). In the remaining groups, it rarely exceeds the aspect of a pit or double 


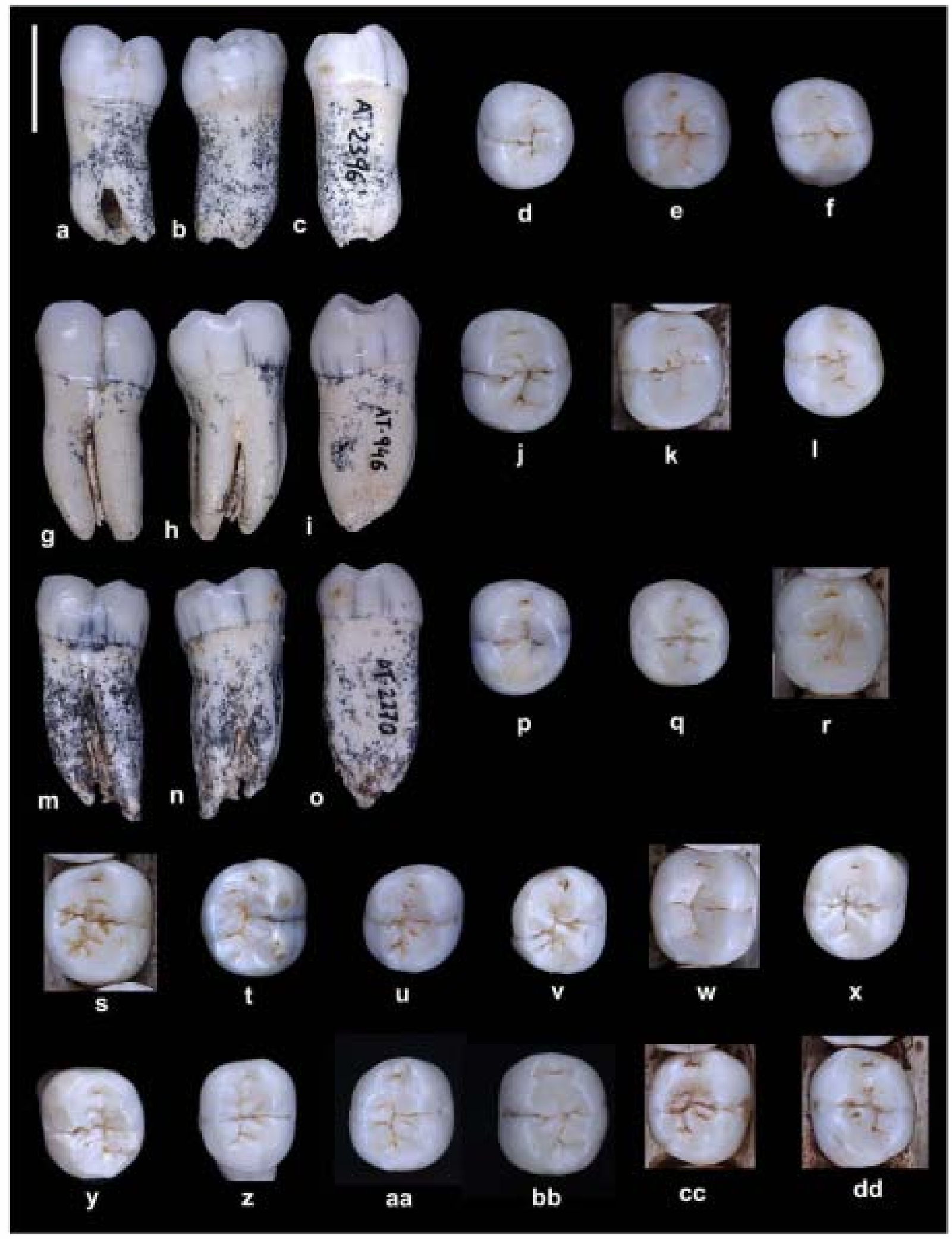

Figure 19. The SH M2s. Buccal (a), lingual (b), mesial (c), and occlusal (d) aspect of AT-2396 (L); occlusal aspect of (e) AT-3179 (L) and (f) AT-273 (L); buccal (g), lingual (h), distal (i), and occlusal (j) aspect of AT-946 (L); occlusal aspect of (k) AT-1 (L) and (I) AT-169 (L); buccal (m), lingual (n), mesial (o), and occlusal (p) aspect of AT-2270 (L); occlusal aspect of ( 1 ) AT-557 (L) and (r) AT-4147 (L); occlusal aspect of s) AT-1 (R); t) AT-142 (R); u) AT-271 (R); v) AT-1761 (R); w) AT-300 (R); x) AT-284 (R); y) AT-2272 (L); z) AT-2193 (R); aa) AT-2763 $(\mathrm{R})$; bb) AT-941 (L); cc) AT-607 (R); and dd) AT-607 (L). Each tooth is positioned so its mesial aspect faces the top of the figure. L: left, R: right. White bar $=1 \mathrm{~cm}$ 
Table 21

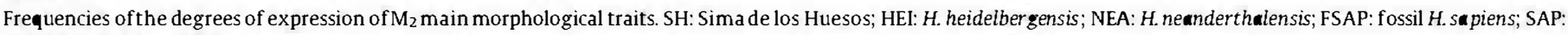
contemporary H. sapiens.

\begin{tabular}{|c|c|c|c|c|c|c|}
\hline $\mathrm{M}_{2}$ & Grade & $\mathrm{SH}$ & HEI & NEA & FSAP & SAP \\
\hline \multirow[t]{4}{*}{ Anterior fovea } & 0 & 0 & $1(12.5 \%)$ & $1(4.5 \%)$ & $12(54.5 \%)$ & $100(64.1 \%)$ \\
\hline & 1 & $5(23.8 \%)$ & 0 & $5(22.7 \%)$ & $5(22.7 \%)$ & $47(30.1 \%)$ \\
\hline & 2 & $16(76.2 \%)$ & $7(87.5 \%)$ & $16(72.7 \%)$ & $5(22.7 \%)$ & $9(5.8 \%)$ \\
\hline & Total & 21 & 8 & 22 & 22 & 156 \\
\hline \multirow[t]{4}{*}{ Middle rigonid crest } & 0 & 0 & $1(12.5 \%)$ & 0 & $17(73.9 \%)$ & $126(82.4 \%)$ \\
\hline & 1 & $2(9.5 \%)$ & $4(50 \%)$ & $6(26.1 \%)$ & $6(26.1 \%)$ & $26(17)$ \\
\hline & 2 & $19(90.5 \%)$ & $3(37.5 \%)$ & $17(73.9 \%)$ & 0 & $1(0.7 \%)$ \\
\hline & Total & 21 & 8 & 23 & 23 & 153 \\
\hline \multirow[t]{4}{*}{ Distal trigonid crest } & 0 & $16(76.2 \%)$ & $5(55.5 \%)$ & $9(40.9 \%)$ & $18(75 \%)$ & $134(88.7 \%)$ \\
\hline & 1 & $5(23.8 \%)$ & $3(33.3 \%)$ & $11(50 \%)$ & $5(20.8 \%)$ & $8(5.3 \%)$ \\
\hline & 2 & 0 & $1(11.1 \%)$ & $2(9.1 \%)$ & $1(42 \%)$ & $9(6 \%)$ \\
\hline & Total & 21 & 9 & 22 & 24 & 151 \\
\hline \multirow[t]{4}{*}{ Deflecting wrinkle } & 0 & $19(90.5 \%)$ & $7(77.7 \%)$ & $17(73.9 \%)$ & $18(78.3 \%)$ & $121(84 \%)$ \\
\hline & 1 & $2(9.5 \%)$ & 0 & $3(13 \%)$ & $4(17.4 \%)$ & $11(7.6 \%)$ \\
\hline & 2 & 0 & $2(22.2 \%)$ & $3(13 \%)$ & $1(4.3 \%)$ & $12(8.3 \%)$ \\
\hline & Total & 21 & 9 & 23 & 23 & 144 \\
\hline \multirow[t]{7}{*}{ Hypoconulid size } & 0 & $6(28.6 \%)$ & 0 & $2(14.2 \%)$ & $16(64 \%)$ & $117(74.5 \%)$ \\
\hline & 1 & $1(48 \%)$ & $1(11.1 \%)$ & 0 & $1(4 \%)$ & $3(1.9 \%)$ \\
\hline & 2 & $7(33.3 \%)$ & 0 & $3(12.5 \%)$ & $1(4 \%)$ & $13(8.3 \%)$ \\
\hline & 3 & $6(28.6 \%)$ & $2(22.2 \%)$ & $6(25 \%)$ & $5(20 \%)$ & $5(3.2 \%)$ \\
\hline & 4 & $1(4.8 \%)$ & $1(11.1 \%)$ & $9(37.5 \%)$ & $1(4 \%)$ & $13(8.3 \%)$ \\
\hline & 5 & 0 & $5(55.5 \%)$ & $4(16.6 \%)$ & $1(4 \%)$ & $6(3.8 \%)$ \\
\hline & Total & 21 & 9 & 24 & 25 & 157 \\
\hline \multirow[t]{5}{*}{ C6 size } & 0 & $12(57.1 \%)$ & $3(33.3 \%)$ & $11(45.8 \%)$ & $19(79.2 \%)$ & $143(94.7 \%)$ \\
\hline & $\mathbf{t}$ & 0 & $2(22.2 \%)$ & $4(16.7 \%)$ & $1(4.2 \%)$ & $4(2.6 \%)$ \\
\hline & 2 & $5(23.8 \%)$ & $3(33.3 \%)$ & $4(16.7 \%)$ & $1(4.2 \%)$ & $3(2 \%)$ \\
\hline & 3 & $4(19.1 \%)$ & $1(11.1 \%)$ & $5(20.8 \%)$ & $3(12.5 \%)$ & $1(0.7 \%)$ \\
\hline & Total & 21 & 9 & 24 & 24 & 151 \\
\hline \multirow[t]{6}{*}{ C7 size } & 0 & $8(4 \%)$ & $4(50 \%)$ & $7(30.4 \%)$ & $19(76 \%)$ & $139(89.7 \%)$ \\
\hline & 1 & $1(5 \%)$ & 0 & $3(13 \%)$ & $1(4 \%)$ & $5(3.2 \%)$ \\
\hline & 2 & $6(30 \%)$ & $1(12.5 \%)$ & $7(30.4 \%)$ & $4(16 \%)$ & $6(3.9 \%)$ \\
\hline & 3 & $3(15 \%)$ & $2(25 \%)$ & $6(26.1 \%)$ & $1(4 \%)$ & $4(2.6 \%)$ \\
\hline & 4 & $2(10 \%)$ & $1(12.5 \%)$ & 0 & 0 & $1(0.6 \%)$ \\
\hline & Total & 20 & 8 & 23 & 25 & 155 \\
\hline \multirow[t]{3}{*}{ Groove pattern } & 1 & $9(40.9 \%)$ & $4(44.4 \%)$ & $17(70.8 \%)$ & $9(34.6 \%)$ & $41(26.5 \%)$ \\
\hline & 2 & $13(59.1 \%)$ & $5(55.5 \%)$ & $7(29.2 \%)$ & $17(65.4 \%)$ & $114(73.5 \%)$ \\
\hline & Total & 22 & 9 & 24 & 26 & 155 \\
\hline \multirow[t]{9}{*}{ Protostylid } & 0 & $11(52.4 \%)$ & $9(100 \%)$ & $17(77.3 \%)$ & $19(86.4 \%)$ & $136(88.3 \%)$ \\
\hline & I & $10(47.6 \%)$ & 0 & $5(22.7 \%)$ & $2(9.1 \%)$ & $17(11 \%)$ \\
\hline & 2 & 0 & 0 & 0 & $1(4.5 \%)$ & 0 \\
\hline & 3 & 0 & 0 & 0 & 0 & 0 \\
\hline & 4 & 0 & 0 & 0 & 0 & $1(0.6 \%)$ \\
\hline & 5 & 0 & 0 & 0 & 0 & 0 \\
\hline & 6 & 0 & 0 & 0 & 0 & 0 \\
\hline & 7 & 0 & 0 & 0 & 0 & 0 \\
\hline & Total & 21 & 9 & 22 & 22 & 154 \\
\hline
\end{tabular}

furrow (grade 2 ), with only two contemporary $H$. sapiens and one Neanderthal from Krapina reaching a Carabelli's complex $\geq 6$. The parastyle is even less frequent (Table 13). With the exception of a grade 5 in $\mathrm{H}$. sapiens, we do not find any case of clear parastyle expression.

In sum, we could confirm that $\mathrm{M}^{2}$ morphology is more variable than $\mathrm{M}^{1}$ and directly influenced by the dental reduction process that affects later Homo species (Wolpoff, 1971; Bermúdez de Castro, 1993; Bermúdez de Castro and Nicolás, 1995), being particularly pronounced in the SH-Atapuerca sample. The MDS plot for the $\mathbf{M}^{2}$ (SOM Fig. 7) reveals that the distributions of $H$. heidelbergensis and SH are generally coincident with that of $H$. neanderthalensis, with the exception of 1 out of $3 \mathrm{H}$. heidelbergensis specimens (Arago 14) and 1 out of the $18 \mathrm{SH}$ specimens included in this analysis.

Upper third molars $\left(M^{3}\right)$

In all of the studied groups, the $\mathbf{M}^{3}$ metacone has undergone significant reduction with regard to the $\mathrm{M}^{1}$ and $\mathrm{M}^{2}$ (Table 14). This fact is particularly evident in the SH sample, where this cusp never exceeds a grade 3 , and, in a high proportion (71.4\%), it is present only as a small cuspule (grade 2). In $H$. neanderthalensis and contemporary $H$. sapiens, we find a few exceptional cases where the metacone is large, but grade 5 is absent in the entire studied sample. In two contemporary $H$. sapiens, one SH individual, and one Neanderthal from Krapina, we find unusual cases where this cusp is only expressed as a faint ridge. The hypocone is also significantly reduced compared to the mesial elements of the molar series (Table 14). The number of cases where this cusp is absent or expressed as a faint ridge (grades 0 and 1 ) ranges from $100 \%$ in H. heidelbergensis (in both Steinheim and Pontnewydd) and 95.2\% in $\mathrm{SH}$, to $30 \%, 35.5 \%$, and $35.2 \%$ in fossil $\mathrm{H}$. sapiens, contemporary $H$. sapiens, and $H$. neanderthalensis, respectively. As mentioned above, the pattern of dental reduction associated with the loss of the main cusps is in line with the pattern of reduction that characterizes late Homo species, and is particularly pronounced in the SH population (Bermúdez de Castro and Nicolás, 1995).

The C5 is absent or only expressed as a faint ridge in the totality of the $\mathrm{SH}$ and $\mathrm{H}$. heidelbergensis samples and in about half of the $H$. neanderthalensis and $H$. sapiens groups (Table 14). In the latter, the range of expression of this trait is wider, and we can also find large C5s (grade 4) in variable frequencies (29.4\% and 20\% in 


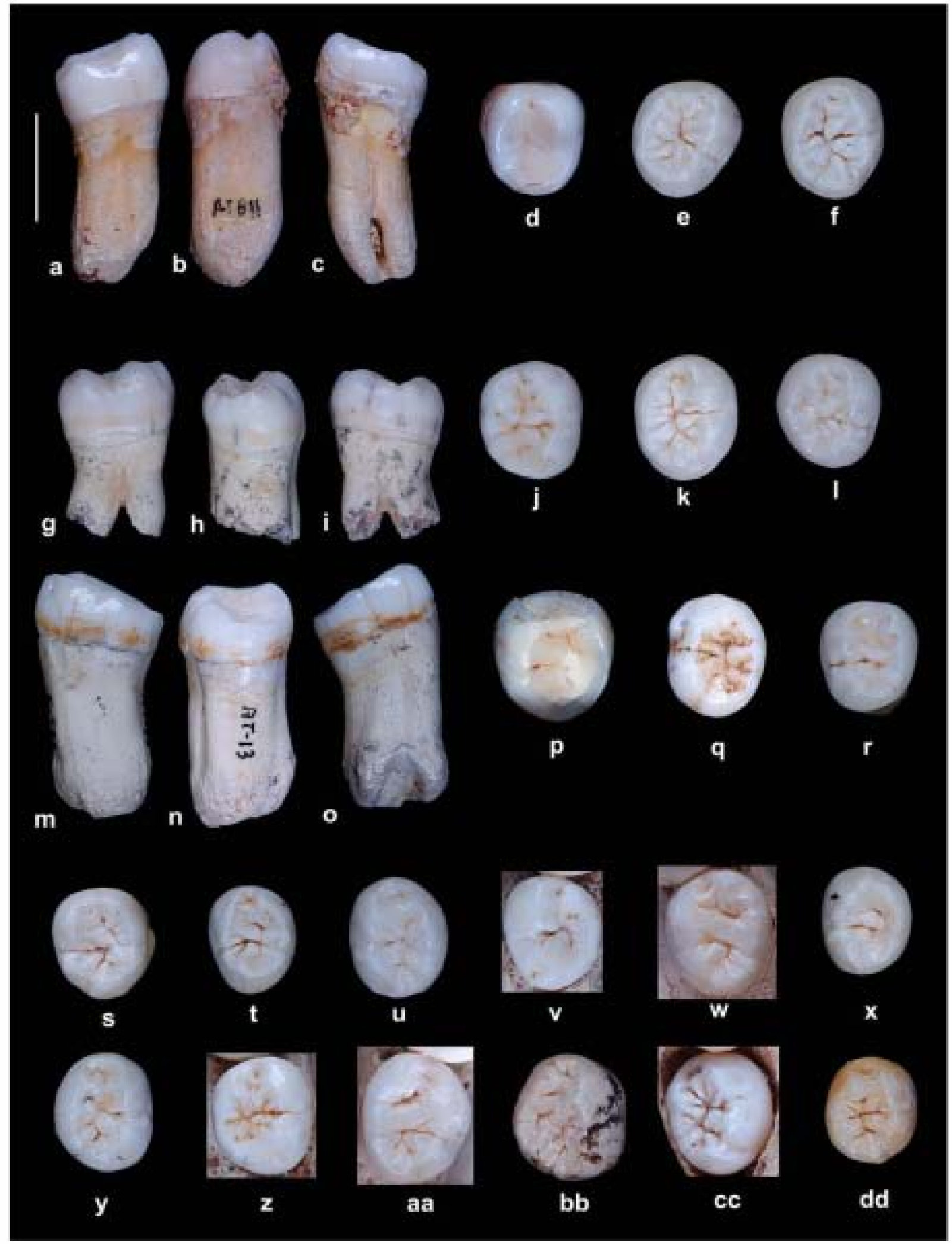

Figure 20. The $\mathrm{SH} \mathrm{M}_{3}$ s. Buccal (a), mesial (b), lingual (c), and occlusal (d) aspect of AT-811 (R); occlusal aspect of (e) AT-222 (R) and (f) AT- 143 (R); buccal (g), distal (h), lingual (i), and occlusal $(\mathrm{j})$ aspect of AT-30 (R); occlusal aspect of (k) AT-1468 (R) and (I) AT-1959 (R); buccal (m), mesial (n), lingual (o), and occlusal (p) aspect of AT-13 (L); occlusal aspect of 9$)$ AT-1473 (L); r) AT-1945 (L); s) AT-2273 (L); t) AT-2760 (L); u) AT-2777 (L); v) AT-1 (L);w) AT-4147 (L); x) AT-2385 (L); v) AT-3182 (R); z) AT-1 (R); aa) AT-300 (R); bb) AT-607 (R); cC) AT-2438 (R); and dd) AT-3943 (R). Each occlusal view is positioned so its mesial aspect faces the top of the figure. L: left, R: right. White bar $=1 \mathrm{~cm}$. 
Table 22

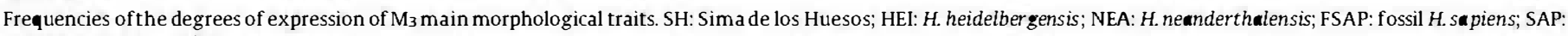
contemporary H. sapiens.

\begin{tabular}{|c|c|c|c|c|c|c|}
\hline $\mathrm{M}_{3}$ & Grade & $\mathrm{SH}$ & HEI & NEA & FSAP & SAP \\
\hline \multirow[t]{4}{*}{ Anterior fovea } & 0 & $1(4.8 \%)$ & $1(20 \%)$ & $2(11.1 \%)$ & $8(47.1 \%)$ & $68(55.7 \%)$ \\
\hline & 1 & $5(23.8 \%)$ & $2(40 \%)$ & $1(5.6 \%)$ & $4(23.5 \%)$ & $36(29.5 \%)$ \\
\hline & 2 & $15(71.4 \%)$ & $2(40 \%)$ & $15(83.3 \%)$ & $5(29.4 \%)$ & $18(148 \%)$ \\
\hline & Total & 21 & 5 & 18 & 17 & 122 \\
\hline \multirow[t]{4}{*}{ Middle rigonid crest } & 0 & $4(18.2 \%)$ & $5(71.4 \%)$ & $1(5.9 \%)$ & $14(93.3 \%)$ & $107(89.9 \%)$ \\
\hline & 1 & $2(9.1 \%)$ & 0 & $4(23.5 \%)$ & $1(6.7 \%)$ & $12(10.1 \%)$ \\
\hline & 2 & $16(72.7 \%)$ & $2(28.6 \%)$ & $12(70.6 \%)$ & 0 & 0 \\
\hline & Total & 22 & 7 & 17 & 15 & 119 \\
\hline \multirow[t]{4}{*}{ Distal trigonid crest } & 0 & $7(30.4 \%)$ & $2(28.6 \%)$ & $10(62.5 \%)$ & $10(62.5 \%)$ & $90(75 \%)$ \\
\hline & $\mathbf{1}$ & $3(13 \%)$ & $4(57.1 \%)$ & $3(18.8 \%)$ & $4(25 \%)$ & $21(17.5 \%)$ \\
\hline & 2 & $13(56.5 \%)$ & $1(143 \%)$ & $3(18.8 \%)$ & $2(12.5 \%)$ & $9(7.5 \%)$ \\
\hline & Total & 23 & 7 & 16 & 16 & 120 \\
\hline \multirow[t]{4}{*}{ Deflecting wrinkle } & 0 & $21(95.5 \%)$ & $5(83.3 \%)$ & $14(93.3 \%)$ & $13(86.7 \%)$ & $101(86.3 \%)$ \\
\hline & 1 & $1(4.5 \%)$ & $1(16.7 \%)$ & $1(6.7 \%)$ & $1(6.7 \%)$ & $7(6 \%)$ \\
\hline & 2 & 0 & 0 & 0 & $1(6.7 \%)$ & $9(7.7 \%)$ \\
\hline & Total & 22 & 6 & 15 & 15 & 117 \\
\hline \multirow[t]{7}{*}{ Hypoconulid size } & 0 & $6(26.1 \%)$ & 0 & $2(12.5 \%)$ & $6(33.3 \%)$ & $63(50 \%)$ \\
\hline & 1 & $2(8.7 \%)$ & 0 & 0 & $1(5.6 \%)$ & $3(2.4 \%)$ \\
\hline & 2 & $4(17.4 \%)$ & 0 & $1(6.3 \%)$ & $2(11.1 \%)$ & $14(11.1 \%)$ \\
\hline & 3 & $6(26.1 \%)$ & $4(57.1 \%)$ & $7(43.8 \%)$ & $6(33.3 \%)$ & $17(13.5 \%)$ \\
\hline & 4 & $4(17.4 \%)$ & $2(28.6 \%)$ & $5(31.3 \%)$ & $2(11.1 \%)$ & $17(13.5 \%)$ \\
\hline & 5 & $1(4.3 \%)$ & $1(143 \%)$ & $1(6.3 \%)$ & $1(5.6 \%)$ & $12(9.5 \%)$ \\
\hline & Total & 23 & 7 & 16 & 18 & 126 \\
\hline \multirow[t]{5}{*}{ C6 size } & 0 & $14(63.6 \%)$ & $3(42.9 \%)$ & $5(33.3 \%)$ & $12(66.7 \%)$ & $94(77.7 \%)$ \\
\hline & $t$ & $1(4.5 \%)$ & $2(28.6 \%)$ & $1(6.7 \%)$ & 0 & $9(7.4 \%)$ \\
\hline & 2 & $6(27.3 \%)$ & $1(14.3 \%)$ & $4(26.7 \%)$ & $4(22.2 \%)$ & $11(9.1 \%)$ \\
\hline & 3 & $1(4.5 \%)$ & $1(143 \%)$ & $5(33.3 \%)$ & $2(11.1 \%)$ & $7(5.8 \%)$ \\
\hline & Total & 22 & 7 & 15 & 18 & 121 \\
\hline \multirow[t]{6}{*}{ C7 size } & 0 & $4(19 \%)$ & $4(66.7 \%)$ & $8(53.5 \%)$ & $13(76.5 \%)$ & $98(79 \%)$ \\
\hline & 1 & $1(4.8 \%)$ & $2(33.3 \%)$ & $1(6.7 \%)$ & $1(5.9 \%)$ & $10(8.1 \%)$ \\
\hline & 2 & $7(33.3 \%)$ & 0 & $3(20 \%)$ & $3(17.6 \%)$ & $6(48 \%)$ \\
\hline & 3 & $7(33.3 \%)$ & 0 & $1(6.7 \%)$ & 0 & $7(5.6 \%)$ \\
\hline & 4 & $2(9.5 \%)$ & 0 & $2(13.3 \%)$ & 0 & $3(2.4 \%)$ \\
\hline & Total & 21 & 6 & 15 & 17 & 124 \\
\hline \multirow[t]{3}{*}{ Groove pattern } & 1 & $4(23.5 \%)$ & $1(143 \%)$ & $9(60 \%)$ & $2(143 \%)$ & $21(19.1 \%)$ \\
\hline & 2 & $13(76.5 \%)$ & $6(85.7 \%)$ & $6(40 \%)$ & $12(85.7 \%)$ & 89 (80.9\%) \\
\hline & Total & 17 & 7 & 15 & 14 & 110 \\
\hline \multirow[t]{9}{*}{ Protostylid } & 0 & $10(47.6 \%)$ & $6(85.7 \%)$ & $8(61.5 \%)$ & $15(88.2 \%)$ & $105(88.2 \%)$ \\
\hline & $\mathrm{T}$ & $4(19 \%)$ & $1(143 \%)$ & $3(23.1 \%)$ & $1(5.9 \%)$ & $9(7.6 \%)$ \\
\hline & 2 & $1(4.8 \%)$ & 0 & $2(15.4 \%)$ & $1(5.9 \%)$ & 0 \\
\hline & 3 & $4(19 \%)$ & 0 & 0 & 0 & $2(1.7 \%)$ \\
\hline & 4 & $1(4.8 \%)$ & 0 & 0 & 0 & $1(0.8 \%)$ \\
\hline & 5 & 0 & 0 & 0 & 0 & $1(0.8 \%)$ \\
\hline & 6 & $1(4.8 \%)$ & 0 & 0 & 0 & $1(0.8 \%)$ \\
\hline & 7 & 0 & 0 & 0 & 0 & 0 \\
\hline & Total & 21 & 7 & 13 & 17 & 119 \\
\hline
\end{tabular}

Neanderthals and fossil $H$. sapiens, respectively). In later Homo species, the $\mathbf{M}^{3}$ tends to show a single accessory cusp in the distal margin, in contrast to Australopithecus and early Homo species where multiple distoconules can be observed (e.g., Moggi-Cecchi et al., 2006, 2010; Martinón-Torres et al., 2008).

While in the $\mathrm{M}^{1} \mathrm{~s}$ the expression of a continuous crista obliqua was the norm, in the $\mathrm{M}^{3} \mathrm{~s}$ we see the opposite. A large majority of the specimens studied here do not express this enamel bridge; it is only present in $5 \%$ of the $H$. neanderthalensis sample (e.g., Krapina M and Saint-Césaire), 20\% of the early $H$. sapiens group, and $25.8 \%$ of the contemporary assemblage (Table 14). The absence of crista obliqua is related to the frequent atypical arrangement of the cusps in the reduced $\mathrm{M}^{3} \mathrm{~s}$ that prevents proper contact between the protocone and the metacone. The transverse crest is also rare (Table 14 ), being only present in $5 \%, 7 \%, 22.2 \%$, and $22.6 \%$ of the $\mathrm{SH}$, $H$. neanderthalensis, fossil $H$. sapiens, and contemporary $H$. sapiens samples, respectively.

Carabelli's trait is uncommon in the $\mathrm{M}^{3}$ s included in this study (Table 14). It is mostly absent in all the groups (particularly in $\mathrm{SH}$, $H$. heidelbergensis, and $H$. neanderthalensis with percentages $\geq 80 \%$, and only in a few cases is expressed as a cusp from small to large. The parastyle is also rare in our sample (Table 14). Contemporary $\mathrm{H}$. sapiens present a wider range of expression for this trait but it is absent in $92 \%$ of the sample. However, the highly crenulated surface of some of the specimens precludes its identification. Finally, the marginal accessory tubercles of the mesial ridge are present in a large majority of the groups, especially in $\mathrm{SH}$, $H$. heidelbergensis, and $H$. neanderthalensis (Table 14), although the crenulations of the enamel surface tend to mask the identification of these tubercles.

In general, we could affirm that the dental reduction processes that generally affect late Homo groups (Wolpoff, 1971; Wood and Abbot, 1983; Bermúdez de Castro, 1993; Bermúdez de Castro and Nicolás, 1995) are evident in our sample, where it is common to find atrophied crowns with atypical arrangements and notable reduction of the main cusps.

The MDS plot for the $\mathrm{M}^{3}$ morphology (SOM Fig. 8) shows that the SH hominins and the two H. heidelbergensis specimens (Steinheim and Pontnewydd) fall within $H$. neanderthalensis variation. As seen in the 95\% confidence intervals, $H$. neanderthalensis presents a wider morphological variation for this tooth class than does the SH group. 
Table 23

MMD distances between each hominin group based on the 31 dental trait MMD analysis.

\begin{tabular}{llllll}
\hline & \multicolumn{1}{c}{ SH } & \multicolumn{1}{c}{ HEI } & NEA & FSAP & SAP \\
\hline SH & 0 & & & & \\
HEI & $0.173322^{\mathrm{a}}$ & 0 & & & \\
NEA & $0.137352^{\mathrm{a}}$ & $0.473684^{\mathrm{a}}$ & 0 & & \\
FSAP & $\mathbf{0 . 4 4 3 9 4 7 ^ { \mathrm { a } }}$ & 0.22426 & $0.474276^{\mathrm{a}}$ & 0 & \\
SAP & $1.008724^{\mathrm{a}}$ & $0.429023^{\mathrm{a}}$ & $\mathbf{1 . 0 8 0 1 9 9}^{\mathrm{a}}$ & $\mathbf{0 . 1 4 2 3 8 4 ^ { \mathrm { a } }}$ & $\mathbf{0}$ \\
\hline
\end{tabular}

${ }^{\text {a }}$ Denotes statistical significance $(\alpha=\mathbf{0 . 0 2 5})$.

\section{Lower central incisor $\left(I_{1}\right)$}

The SH group exhibits more pronounced curvatures, with the totality of the sample ranging from weak to moderate convexities (Table 15). The convexity is also moderate in two out of three Arago specimens, whereas in Mauer and Arago 30 this trait does not exceed a grade 1 . In $H$. neanderthalensis, only $33.3 \%$ of the sample presents more than traces of convexity (grade 2 ). This proportion is identical to that found in the fossil $H$. sapiens sample, where the Dolní Věstonice specimens and one of the Pavlov $\mathrm{I}_{1} s$ display weak to moderate curvatures. Only both $H$. sapiens samples contain specimens with completely flat labial surfaces.

The expression of the tuberculum dentale presents little variation (Table 15). With only two exceptions from the fossil $H$. sapiens group, the lingual surface of this tooth is always smooth or presents a somewhat inflated basal eminence that is continuous with the essential ridge.

Shovel shape is also an uncommon trait in this tooth class (Table 15), and with only one exception from the contemporary $H$. sa piens group, it never exceeds grade 2 . In the vast majority of the studied groups, shoveling is completely absent or expressed as faint ridges only noticeable on palpation.

In general, $\mathrm{I}_{1} \mathrm{~S}$ are teeth with low intra- and inter-population variability. The $\mathrm{SH} \mathrm{I}_{1} \mathrm{~S}$ are characterized as relatively massive, due to their large buccolingual diameters (Bermúdez de Castro, 1986; Table 6). They also display a moderate labial convexity that may contribute to a false appearance of shovel shape. The "particularity" of the Middle Pleistocene hominin and Neanderthal $\mathrm{I}_{1} \mathrm{~s}$ may rely

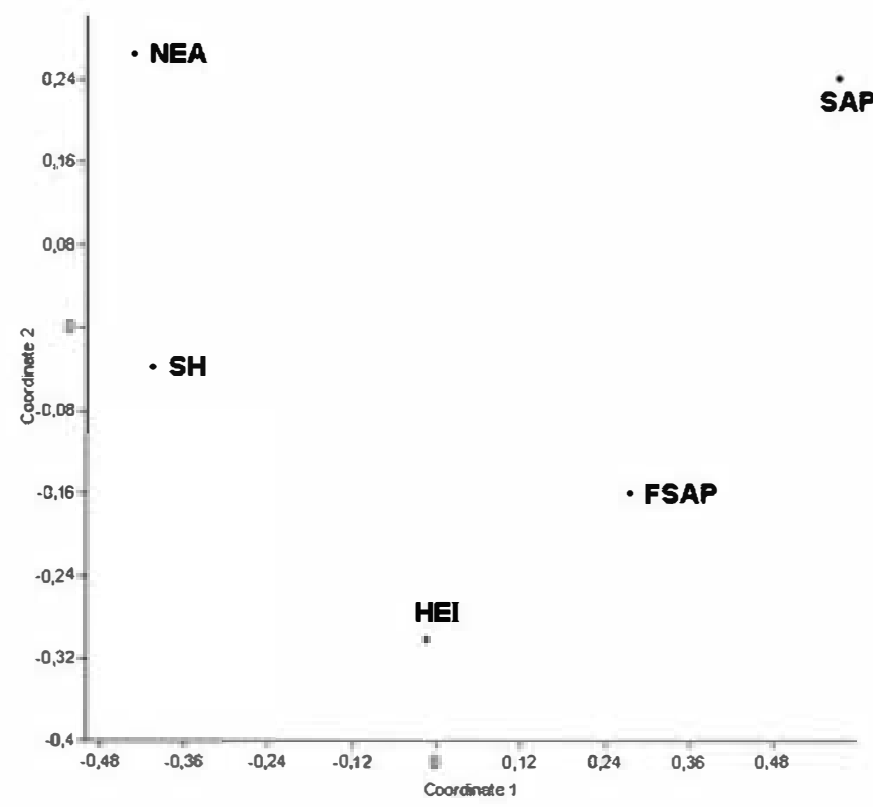

Figure 21. MDS plot obtained from the phenetic analysis of the dental evidence. SH: Sima de los Huesos; HEI: $H$. heidelbergensis; NEA: $H$. neanderthalensis; FSAP: fossil H. supiens; SAP: contemporary H. supiens. more on metric, rather than morphological, features related to anterior teeth expansion, which characterizes these populations until 100 ka (e.g., Wolpoff, 1971; Bermúdez de Castro, 1986; Rosas and Bermúdez de Castro, 1998).

The MDS plot regarding $I_{1}$ morphology illustrates the low intraand inter-population variability of this tooth, with a clear overlap of specimens and distributions (SOM Fig. 9).

\section{Lower lateral incisors $\left(I_{2}\right)$}

The $I_{2} S$ are, in general, more convex than the $I_{1} s$, and flat labial surfaces are exclusive to $H$. sapiens $(62.5 \%$ in fossil $H$. sapiens and $60.3 \%$ in contemporary specimens) (Table 16). With only one exception from Arago, the maximum convexity in our samples never exceeds a grade 3 , and the highest frequencies of this grade are the ones displayed by $\mathrm{SH}(47.4 \%)$.

The tuberculum dentale is uncommon in our sample (Table 16), and most of the $\mathrm{I}_{2} \mathrm{~S}$ present a smooth lingual surface or a basal eminence that is continuous with the essential ridge.

We have not found specimens with pronounced shovel shapes in our sample (Table 16). The SH population presents the highest degrees of light shoveling $(63.2 \%$ presents grade $\geq 2)$, while contemporary $H$. sapiens show the highest number of non-shoveled I2s. Within the $H$. heidelbergensis sample, the trait is absent in Mauer but present in Arago. In Neanderthals and early $\mathrm{H}$. sapiens, the ridges are only present in half of the specimens.

The MDS plot (SOM Fig. 10) shows the higher morphological variability of the $I_{2} S$ compared to the $I_{1} s$. With only two exceptions, all of the $\mathrm{SH}_{2} \mathrm{~S}$ fall within the morphological variability of $H$. neanderthalensis. One of the $H$. heidelbergensis specimens (Mauer) also falls within both $\mathrm{SH}$ and $H$. neanderthalensis 95\% confidence intervals, whereas Arago 97 falls outside of the variability of both groups.

\section{Lower canines $(C$,}

The tuberculum dentale is present in approximately half of the $\mathrm{SH}$, Neanderthal, and fossil $\mathrm{H}$. sapiens samples. We rarely find more than ridge traces or grade $3(5.9 \%$ and $0.6 \%$ in fossil and contemporary $H$. sapiens, respectively). None of the five $H$. heidelbergensis specimens analyzed express this trait (Table 17).

The expression of shovel shape in this tooth class ranges from slight to pronounced (Table 17). $\mathrm{H}$. sapiens is the only group containing specimens with a grade 0 . More than $60 \%$ of the $\mathrm{SH}$, $H$. heidelbergensis, and $H$. neanderthalensis groups present moderate to conspicuous marginal ridges.

With five exceptions from the Neanderthal group (two from Cabezo Gordo and one each from Saint-Césaire, Arcy Renne, and Monsempron) and one from the contemporary H. sapiens group, where the mesial marginal ridge is more prominent than the distal one, the mesial canine ridge is absent (Table 17).

The distal accessory ridge is expressed in variable percentages among the studied groups (Table 17). H. neanderthalensis is the group with higher frequencies and more pronounced forms. Within the $H$. heidelbergensis sample, it is only present in Mauer and Arago 34. In the fossil and contemporary $H$. sapiens groups, it is expressed in $41.6 \%$ and $23.3 \%$, respectively, and in the SH group, only $25 \%$ of the specimens present this trait and always in its mild form (grade 1).

Apart from these traits, we also notice that the essential ridge of the lower canines tends to be flat and smooth. In some specimens like Mauer or Arago 24, it is relatively more conspicuous and swollen, although it never reaches the degree of development that is typical of Australopithecus and early Homo (Martinón-Torres et aI., 2007b, 2008). None of them develop a buccal cingulum, despite the expression of certain buccal bulging typical of the Arago and some 
SH specimens. The $\mathrm{C}$, morphology of the late Homo groups is very incisor-like, and aside from certain variation in the frequencies and degree of shoveling, it does not provide significant taxonomicdiscriminative information.

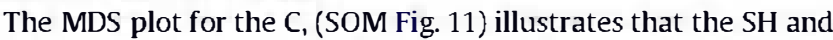
$H$. heidelbergensis morphology is contained in the $H$. neanderthalensis distribution, with the latter showing higher morphological variation.

\section{Lower third premolar $\left(P_{3}\right)$}

Homo species can express a variable number of accessory lingual cusps adjacent to the metaconid. These cusps tend to develop as ridges or tubercles rather than as independent cusps with a free apex (e.g., Tobias, 1991; Martinón-Torres, 2006). The development of extralingual cusps, usually located in the distolingual talonid, may contribute to the asymmetry of the occlusal outline of the tooth (Wood and Uytterschaut, 1987; Gómez-Robles et al., 2008). These cusps are delimited by secondary fissures that stem out from the posterior fovea. In the extreme of this variation, we can find $H$. sapiens where only $35.3 \%$ and $57.9 \%$ of the contemporary and fossil groups, respectively, express at least one accessory lingual cusp (Table 18). Half of the contemporary H. sapiens group do not express a distinct metaconid. A single lingual cusp is expressed in approximately $20 \%$ of each group and this percentage is slightly higher in the Neanderthal sample. In the SH hominins and $H$. heidelbergensis, the most common morphology is a $P_{3}$ with only one lingual cusp and a round distal marginal where no accessory cusps are developed ( $52.9 \%$ and $40 \%$, respectively). In these groups, specimens with more than one accessory cusp are rare. H. neanderthalensis is the group with higher frequencies of two accessory lingual cusps apart from the metaconid (e.g., some of the Arcy Renne specimens, Monsempron, or La Quina 5), but, as mentioned above, they are rarely as pronounced as in early hominins. In the SH hominins, H. heidelbergensis, and H. neanderthalensis, it is common to find a transverse crest connecting the protoconid and the metaconid (Table 18). In contrast, this crest is rare in the contemporary $H$. sapiens sample, where only $12.7 \%$ of specimens show a continuous ridge.

The expression of a distal accessory ridge is relatively more frequent than the expression of a mesial one (Table 18). Mesial accessory ridges are uncommon in all of the studied groups, with $H$. heidelbergensis being the only group with a proportion of more than $20 \%$. The distal accessory ridge is more frequent than the mesial, particularly in $\mathrm{H}$. neanderthalensis (90\%). In the $\mathrm{SH}$ and fossil $H$. sapiens samples, this crest is present in about half of the specimens, while its proportion in the contemporary $H$. sapiens group is only $9.7 \%$. The absence of mesial and distal accessory ridges in contemporary $H$. sapiens contributes to the simplified occlusal surface of this tooth class.

The MDS plot (SOM Fig. 12) shows that all of the SH hominins (with only one exception) fall within the H. neanderthalensis distribution, whereas half of the $H$. heidelbergensis sample fall outside of the $H$. neanderthalensis distribution (but within the 95\% confidence interval for the SH sample).

Single-rooted $\mathrm{LP}_{3} S$ are the rule for late Homo populations, following the trend toward root simplification in the genus Homo (Wood et al., 1988). As exceptions to the norm, double-rooted specimens occur only in Krapina (Kallay, 1963), Arago 13 (Bermúdez de Castro et al., 2003a), and in one specimen from $\mathrm{SH}$ (Martinón-Torres, 2006).

Apart from the differences in the above mentioned traits, there are some shape and morphological features that are important from a taxonomic point of view. Some of these traits are ideally assessed by geometric morphometric analysis (Gómez-Robles et al., 2008).
Late Homo species such as H. sapiens, H. neanderthalensis, and H. heidelbergensis tend to differ from early African and Asian hominins in their substantially symmetrical contours. Among the former, we can also distinguish two slightly different morphotypes, one corresponding to $\mathrm{H}$. sapiens and one corresponding to $H$. heidelbergensis and $H$. neanderthalensis. H. sapiens is characterized by symmetrical and circular outlines with weak or absent talonids. The occlusal polygon (as defined by Martinón-Torres et al. [2006] and Gómez-Robles et al. [2008]) is large and centrally located due to the buccally-displaced protoconid tip. $H$. heidelbergensis and $H$. neanderthalensis are characterized by small occlusal polygons, closely positioned near the lingual margin of the tooth due to the lingual displacement of the protoconid. Their occlusal contour is symmetrical or slightly asymmetrical, frequently with the distal side of the lingual outline being convex and the mesial side being concave. Interestingly, the Arago specimens have been described as somewhat more primitive than other $H$. heidelbergensis and $H$. neanderthalensis specimens, with wider occlusal polygons and more asymmetrical contours (Gómez-Robles et al., 2008; GómezRobles, 2010). This conformation would be in line with the "intermediate" morphologies (between Early and Upper Pleistocene hominins) previously described for the Arago hominins (Bermúdez de Castro et al., 2003a). The SH hominins would fall within H. neanderthalensis, although their morphology is even more pronounced and/or derived than that of the classic Neanderthals, as they fall in the extreme of the $H$. neanderthalensis spectrum of variation. This fact is relevant for the discussion about the neanderthalization process (Dennell et al., 2010), since Middle Pleistocene fossils have been traditionally described as generally "less Neanderthal" than $H$. neanderthalensis (Bermúdez de Castro, 1987, 1988, 1993; Arsuaga et al., 1997c). In addition, the SH $P_{3}$ S exhibit a canine-like aspect with a very reduced and bulbous lingual cusp, as most of $H$. heidelbergensis and $H$. neanderthalensis $P_{3}$ s.

\section{Lower fourth premolar $\left(P_{4}\right)$}

Except for a few H. sapiens specimens, all of the studied species range from one to two or more lingual cusps, including the metaconid. The SH hominins, $H$. heidelbergensis, and $H$. neanderthalensis display the highest frequencies of more than two cusps $(95 \%, 100 \%$, and $88.9 \%$, respectively) (Table 19 ). Similar to what we find in the $P_{3} S$, the morphology of the accessory cusps rarely exceeds that of thickened ridges demarcated by feeble grooves. In fossil H. sapiens, most of the specimens present one or two accessory lingual cusps (55\%) and, in our contemporary $H$. sapiens, the predominant type is the expression of a metaconid plus a distolingual platform (48.6\%). In these groups, the location of the accessory cusps is restricted to the distolingual quarter of the tooth, in contrast to earlier hominins where the talonid also comprises a buccodistal portion (MartinónTorres et al., 2006), commonly demarcated in the external contour of the tooth by a furrow.

A continuous transverse crest connecting both main cusps is the common condition in the $\mathrm{SH}$ hominins and $H$. neanderthalensis (75\% and 69.2\%, respectively). This trait has been previously described as typical of Neanderthals (Bailey, 2002a,b) and less common in other hominin species such as H. sapiens (Table 19). In our sample, contemporary $H$. sapiens only expresses this feature in $8.5 \%$ of the population, although this percentage is higher in the fossil $H$. sapiens sample (41.7\%). In $H$. heidelbergensis, we only find one case where this crest is clearly continuous (Arago 89); in the remaining specimens, the crest is commonly interrupted by the sagittal groove (e.g., Mauer or Pontnewydd 5).

Mesial accessory ridges of the protoconid are uncommon in the whole sample (Table 19). Conversely, distal accessory ridges are relatively common in all of the studied species except for the 
contemporary $H$. sapiens groups (22.3\%). The MDS plot (SOM Fig. 13) shows a complete overlap of $H$. heidelbergensis and $\mathrm{SH}$ distributions within the $95 \%$ confidence interval of $H$. neanderthalensis.

Finally, $\mathrm{P}_{4}$ shape has been previously assessed by means of geometric morphometrics (Martinón-Torres et al., 2006), revealing that $H$. heidelbergensis (including $\mathrm{SH}$ ) and $H$. neanderthalensis have fixed plesiomorphic traits in high percentages, such as an asymmetrical contour, mesial metaconid, and a transverse crest (see also Bailey and Lynch, 2005), which are also frequent in Asian H. erectus and H. antecessor (Martinón-Torres et al., 2007a,b). The main difference compared to earlier hominins is in the loss of the buccodistal portion of the talonid and a reduction of the lingual half of the tooth, which is in turn related to the dental reduction processes that affect late Homo species (Bermúdez de Castro and Nicolás, 1995). This process is particularly observed in the $\mathrm{P}_{4} \mathrm{~S}$ of $H$. antecessor, $H$. heidelbergensis, and $H$. neanderthalensis. $H$. sapiens evolved a derived form, with a symmetrical outline, a centered metaconid, and a significant reduction of the talonid (MartinónTorres et al., 2006).

\section{Lower first molar $\left(M_{1}\right)$}

As mentioned above, the SH hominins are characterized by the almost constant presence of a short and deep anterior fovea (Table 20). In only $15 \%$ of the sample, this fovea is slight or linear and open toward the mesial marginal ridge in the shape of a triangular depression, matching the description of the classic anterior fovea defined by Hrdlička (1924) and Scott and Turner (1997). The type of pit-like and closed fovea identified in the SH hominins has been described as typical of $H$. neanderthalensis (Bailey, 2002a,b; Martinón-Torres et al., 2007a,b), presenting similarly high percentages as the SH sample in our study (81.3\%). In $H$. heidelbergensis, the frequencies of a grade 2 anterior fovea are lower (57.1\%), being absent in the holotype of the species, Mauer, and slight or linear in Saint-Césaire, Arago 89, and Pontnewydd 11. The anterior fovea is only present in $38.9 \%$ and $21.1 \%$ of the fossil and contemporary $H$. sapiens samples, respectively, with a few exceptions ( 2 out of 114 specimens in the contemporary group and Qafzeh $\mathrm{H} 4$ from the fossil $H$. sapiens group) where it adopts a pitlike aspect.

This type of fovea is commonly combined with the expression of a continuous middle trigonid crest that borders the fovea distally (Table 20). The concomitant expression of both features has been considered typical of Neanderthals (Zubov, 1992a,b; Bailey, 2002a,b; Bailey et al., 2011) and European Middle Pleistocene populations (Martinón-Torres et al., 2007a,b), although it can also be found in Asian $H$. erectus from Java in lower percentages (Martinón-Torres, 2006; Martinón-Torres et al., 2007a,b). In our sample, $H$. neanderthalensis expresses a continuous crest in $53.3 \%$ of cases and it is concomitant with a pit-like anterior fovea in $46.4 \%$ of the specimens. Mountmaurin and 3 out of 4 Arago individuals present this Neanderthal-like combination, whereas in Mauer, despite being the holotype, it is absent (Martinón-Torres, 2006; Bailey et al., 2011). In the SH sample, the middle trigonid crest is continuous in 95\% of the specimens, and its expression is concomitant with a grade 2 anterior fovea in $85 \%$ of the sample. Only one specimen from the contemporary $H$. sapiens group and one from the fossil $\mathrm{H}$. sapiens sample express a continuous middle trigonid crest.

The distal trigonid crest is an uncommon trait in all the studied groups, being only present in $30 \%, 35 \%$, and $12.7 \%$ of $\mathrm{SH}$, and fossil and contemporary $H$. sapiens samples, respectively (Table 20). In $H$. heidelbergensis, half of the sample expresses it in a grade 1 , in the shape of a prolongation of the accessory distal ridge of the protoconid, delimited by a short marginal groove (e.g., Mountmaurin and Arago 13). More than half of the H. neanderthalensis sample do not express it, but in $14.3 \%$ of this species it reaches a grade 2 (e.g., Petit-Puymoyen 211 or Arcy Renne A6), ascertained as a pronounced ridge that either obliterates the central groove or is delimited by a marginal groove that also includes the metaconid (but see Bailey et al., 2011, for other estimations).

The deviation of the metaconid essential ridge, also known as a deflecting wrinkle, is absent in most of the studied specimens (Table 20), although variable percentages of grade 1 and 2 forms can be found in all of the groups. Cases where this crest is clearly Lshaped and connects the metaconid and the hypoconid are rare (below 20\% in the SH hominins, $H$. heidelbergensis, and contemporary $\mathrm{H}$. sapiens, and below $10 \%$ in the rest of the groups).

Invariably, all of the groups present large or very large (grades 4 and 5) hypoconulids (Table 20). Interestingly, SH presents two exceptional cases of four-cusped $M_{1} s$, a circumstance that only happens in small percentages in the $H$. sapiens samples analyzed here. We are certain that these teeth are $\mathrm{M}_{1} \mathrm{~s}$ because we also preserve the $\mathrm{M}_{2} \mathrm{~s}$ of the individuals they belong to.

The frequencies of $C 6$ expression in the $M_{1} s$ are low in all of the studied groups (Table 20), and completely absent in the $\mathrm{SH}$ population. With $20.7 \%, H$. neanderthalensis is the group with the highest percentage of large C6s (e.g., Hortus II or PetitPuymoyen 2).

In our sample, C7s are more frequent than C6s, although they tend to be small (Table 20) and displaced as a bulge on the lingual surface of the metaconid (similar to grade 1A of the ASUDAS classification). Frequencies of large and well-delimited $C 7 \mathrm{~s}$ are usually below $10 \%$ (except for $H$. heidelbergensis, with $16.7 \%$ of specimens with a C7 of grade 4), although, in some exceptional cases, they can displace the metaconid and entoconid (e.g., Dolní Věstonice 15).

Regarding the groove pattern, the majority of the groups present the dryopithecine form, followed by the " $X$ " and the "+ " patterns in that order (Table 20). The proportion of the Y-pattern is higher in both $H$. sapiens samples and the $H$. neanderthalensis sample. In the SH sample, $Y$ and non- $Y$ types are relatively balanced (55.6\% vs. $44.4 \%$, possibly related to the marked posterior dental reduction in this group. Similarly, $42.9 \%$ of the $H$. heidelbergensis sample (Pontnewydd 11, Arago 13, and Arago 89) does not show a dryopithecine type.

In our sample, the protostylid is uncommon (Table 20). Almost all of the specimens range from absence to grade 1 , a degree that is still questioned as a minimum expression of this trait (Scott and Turner, 1997). This feature may be a better populational, rather than taxonomical marker in late Homo species, since it can be present in moderate percentages in some H. sapiens groups (Irish, 1993) but is almost absent in all of the $H$. sapiens specimens analyzed here.

The MDS plot for the $M_{1}$ (SOM Fig. 14) shows that $H$. neanderthalensis covers the morphological variability of the SH sample, whereas 2 out of $6 \mathrm{H}$. heidelbergensis specimens (Mauer and Arago 89), fall outside of the H. neanderthalensis and SH distributions.

\section{Lower second molars $\left(M_{2}\right)$}

Similar to the morphology of the $\mathbf{M}_{1}$ s, deep and short anterior foveae are highly frequent (Table 21 ) in the $\mathrm{SH}, \mathrm{H}$. neanderthalensis, and $H$. heidelbergensis $M_{2} s$ (76.2\%, 72.7\%, and $87.5 \%$, respectively). Around half of the early and contemporary $H$. sapiens samples do not express an anterior fovea and only in small percentages can we find a grade $2(22.7 \%$ and $5.8 \%$, respectively). The expression of an uninterrupted middle trigonid crest is predominant in $\mathrm{SH}(90.5 \%)$ and $H$. neanderthalensis (73.9\%), but is only present in this form in $37.5 \%$ of the $H$. heidelbergensis specimens (Mountmaurin, Arago 6, 
and Arago 10). As with the $\mathrm{M}_{1}$ s, the middle trigonid crest is widely absent in $\mathrm{H}$. sapiens, and, when it is present, it is interrupted by the central groove (Table 21). The concomitant expression of a continuous middle trigonid crest and a grade 2 anterior fovea is frequent in $H$. neanderthalensis (66.6\%) and Sima de los Huesos (71.4\%). It is less common in $H$. heidelbergensis (33.3\%) and H. sapiens (1 out of 173 specimens). This trait combination has been described as typical of $H$. neanderthalensis and European Middle Pleistocene groups (Bailey, 2002a,b; Martinón-Torres et al., 2007a,b), but it is absent in Mauer. The distal trigonid crest is less frequent than its mesial counterpart, being mostly absent in all groups except for the H. neanderthalensis sample, where grades 1 and 2 are slightly more frequent than grade 0 (Table 21). This feature in its maximum degree of expression is absent in the $\mathrm{SH} \mathrm{M}_{2}$ s. The deflecting wrinkle is also rare in our sample (Table 21). The hypoconulid undergoes a significant reduction with regard to the $\mathbf{M}_{1}$ (Tables 20 and 21). Its expression ranges from absent to large, but in the $H$. sapiens samples it is expressed as a moderately large cusp (grade $\geq 3$ ) in only $28 \%$ and $15.3 \%$ of the fossil and contemporary samples, respectively. Four-cusped $\mathrm{M}_{2} \mathrm{~s}$ are rare in the remaining groups (see also Gómez-Robles et al., 2011a) although the hypoconulid is absent or expressed as a vestige (grades 0 and 1 ) in $33.4 \%, 11.1 \%$, and $14.2 \%$ of $\mathrm{SH}, H$. heidelbergensis, and $H$. neanderthalensis samples, respectively.

The position of the C6 within the occlusal outline is lingual, and, in some cases, it is expressed despite the absence of a hypoconulid (e.g., individuals III and XI from SH and Hortus V). In our sample, this cusp is generally small in all the studied groups (Table 21). The C7 rarely exceeds a grade 2 in our $H$. sapiens sample. In contrast, the $\mathrm{SH}, H$. heidelbergensis, and $H$. neanderthalensis samples display moderate frequencies of independent and well-demarcated $\mathrm{C7} \mathrm{s}$ (grades $\geq 3$ in $25 \%, 37.5 \%$, and $26.1 \%$, respectively) (Table 21 ).

Regarding the groove pattern, "+" patterns are more common than " $X$ " patterns. The latter can be found in the Middle Pleistocene in the SH hominins and some Krapina individuals. In the studied sample, $H$. neanderthalensis is the only group where the Y-pattern prevails over the other two (70.8\%).

The expression of a protostylid is rare in our sample (Table 21), and with only one exception from the contemporary $H$. sapiens group, it never exceeds a grade 2 .

From the MDS plot (SOM Fig. 15) we infer that the SH $\mathrm{M}_{2} \mathrm{~S}$ morphology falls within the $H$. neanderthalensis $95 \%$ confidence interval. In contrast, the $H$. heidelbergensis sample appears more variable than the SH sample, and 2 out of 8 specimens (Mauer and Arago 89) fall outside of both $\mathrm{SH}$ and $H$. neanderthalensis distributions.

Although a consideration of root morphology was not one of the goals of the present study, it was ascertained that more than half of the SH population presents hypotaurodontism, and there are some isolated cases where bif urcations do not occur until the apical third. This morphology would be similar to that of $H$. neanderthalensis roots, which are assumed to present high frequencies of taurodontism (e.g., Kricum et al., 1999; Lebel and Trinkaus, 2002; Kupczik and Hublin, 2010).

\section{Lower third molars $\left(M_{3}\right)$}

The expression of an anterior fovea is considerably less frequent in $H$. sapiens $(44.3 \%$ in the contemporary sample and $53 \%$ in the fossil sample) than in $H$. neanderthalensis (89\%), $H$. heidelbergensis (80\%), and the SH hominins (95.2\%) (Table 22). In H. sapiens as a species, this fovea is usually long and shallow, whereas in the remaining groups it tends to be short and deep. The expression of a middle trigonid crest is considerably more frequent in the $\mathrm{SH}$ and H. neanderthalensis groups, especially in its continuous form (72.7\% and 70.6\%, respectively), while this form is totally absent in the $H$. sapiens samples studied here. As in the $\mathrm{M}_{1} \mathrm{~S}$ and $\mathrm{M}_{2} \mathrm{~S}$, the concomitant expression of a continuous middle trigonid crest and a deep short anterior fovea is also typical of SH and $H$. neanderthalensis ( $57.14 \%$ and $64.7 \%$, respectively). This pattern occurs in 1 out of $5 \mathrm{H}$. heidelbergensis specimens in our study (Mountmaurin) and is absent in our $H$. sapiens sample. This combination can be traced back to Asian $H$. erectus (particularly those from Sangiran locality) (Martinón-Torres, 2006).

The expression of a distal trigonid crest (Table 22) is a common trait within the SH hominins (69.5\%), including its continuous form. It is also relatively frequent in $H$. heidelbergensis but is largely absent in $H$. neanderthalensis and both $H$. sapiens samples.

The deflecting wrinkle is a rare trait in our sample (Table 22). However, its identification may be obscured by the atypical cusp arrangement of this dental class in late Homo species, as a consequence of a strong dental reduction processes. A deflecting wrinkle of grade 2 is only found in one early H. sapiens (Dolní Věstonice 13) and $7.7 \%$ of the contemporary group.

The hypoconulid reduction in the $\mathrm{SH} \mathrm{M}_{3} \mathrm{~S}$ is even more pronounced than in the $\mathrm{M}_{2} \mathrm{~s}$. This cusp is absent or barely expressed as a bif urcation of the distal groove in $39 \%$ of this population, and in $38.9 \%$ and $52.4 \%$ of the fossil and contemporary $H$. sapiens samples, respectively (Table 22). In $H$. neanderthalensis, this percentage is lower (12.5\%). The size of this cusp ranges from medium to small in the studied groups, and in only less than 10\% (except for $H$. heidelbergensis) it reaches the maximum degree of the qualitative scale of expression. Within $H$. heidelbergensis, some specimens like Mauer develop a large hypoconulid, whereas in Arago 13 this cusp is small.

Moderate-sized C6 (grades 2 and 3) are present in more than half of the $H$. neanderthalensis sample, in less than the $35 \%$ of $\mathrm{SH}$ hominins, $H$. heidelbergensis, and early $H$. sapiens, and only in $15 \%$ of the contemporary $H$. sapiens group. There are a few cases where the C6 is expressed in the absence of a hypoconulid (e.g., Krapina G, SH individual XXVI, and two specimens from the contemporary H. sapiens San Nicolás sample).

The $\mathrm{C} 7$ is also highly variable in its expression, ranging from absent to large in all of the groups but $H$. heidelbergensis or early H. sapiens (where it never exceeds a grade 2) (Table 22). SH is the group with the highest frequencies of large $C 7 \mathrm{~s}$, and in many cases this cusp contributes to atypical cusp arrangements that obscure the differentiation and identification of main and accessory cusps. It also affects the identification of the groove pattern (Table 22), which is predominantly non-dryopithecine, with the exception of $H$. neanderthalensis, where a " $\mathrm{Y}$ " arrangement is present in more than half of the sample (60\%). In $H$. sapiens, the predominant pattern is " $X$," whereas in Middle Pleistocene groups the prevailing from is "+."

The protostylid is rare in all of the studied groups, although its expression is more frequent in $M_{3}$ than in $M_{1}$ and $M_{2}$ (Tables 20-22), particularly in the SH population and the contemporary $H$. sapiens group.

From the MDS plot (SOM Fig. 16) we can infer that all the $H$. heidelbergensis and $\mathrm{SH} \mathrm{M}_{3}$ s (with one exception out of 21 specimens) fall within the $H$. neanderthalensis range of variability.

\section{Discussion}

We are aware that dental evidence is only one piece of a complex jigsaw puzzle, and that other types of data should be considered in order to explore the origins of the Neanderthals. Far from intending to answer all the questions about the evolutionary story of Neanderthals, we aim to contribute new evidence to the 
development and testing of hypotheses about a possible "neanderthalization" process.

Several studies have stated that Neanderthals display morphological traits and proportions that are distinctive of this species (e.g., Smith, 1989; Bermúdez de Castro, 1993; Crummett, 1995; Bermúdez de Castro and Nicolás, 1995; Stefan and Trinkaus, 1998; Tattersall and Schwartz, 1999; Bailey, 2002a,b; Gómez-Robles et al., 2007, 2008, 2011). In particular, their dental morphology has been widely analyzed (e.g., Boule, 1923; Keith, 1924, 1925; Krogman, 1927; Weidenreich, 1937; Boule and Vallois, 1957; Patte, 1959), and, beyond the high frequencies of taurodontism (e.g., Keith, 1924; Hillson, 1986; Klein, 1999) or pronounced shovel shape (e.g., Mizoguchi, 1985; Crummett, 1995; Bailey, 2002a), other dental traits have been identified as "unique" to this species (e.g., Zubov, 1992a,b; Bailey, 2002a,b; Martinón-Torres et al., 2007a,b; Gómez-Robles et al., 2007, 2008, 2011; Bailey et al., 2011). However, a true assessment of Neanderthal "uniqueness" and its evolutionary significance requires comparison to European Middle Pleistocene populations, considered by many researchers as the direct ancestors of the classic Neanderthals (e.g., Hublin, 1982, 1984, 1996, 2009; Stringer, 1985, 1993; Arsuaga et al., 1993, 1997c). In this context, the Sima de los Huesos dental sample has enriched the European Pleistocene fossil record in an extraordinary manner, representing more than the $90 \%$ of the Middle Pleistocene worldwide human fossil record and becoming a crucial sample for understanding a possible neanderthalization process.

Such a large sample as Sima de los Huesos allows us to define the dental morphological pattern of a Middle Pleistocene population. In sum, we can state that the $\mathrm{SH} \mathrm{I}^{1}$ s are characterized by a pronounced labial convexity, moderate shovel shape, and prominent basal eminence over which a light tubercle may develop. The $\mathrm{SH} \mathrm{I}^{2} \mathrm{~S}$ also display conspicuous labial convexity and a type of shovel shape we have coined "triangular shovel shape" that is exclusive of European Middle Pleistocene and Neanderthal populations (although incipient forms of it can be traced back to $H$. antecessor and Asian H. erectus species) (Martinón-Torres et al., 2007a,b; Gómez-Robles, 2010). With the only exception of Qafzeh, which present pronounced incisor convexity and incipient triangular shovel shape, this morphology is absent in $H$. sapiens. Upper canines display moderate to pronounced degrees of shovel shape, prominent lingual tubercles, and high frequencies of mesial ridges, overlapping with Neanderthal groups in their distributions. SH sample coincides with $H$. neanderthalensis in the expression of "mass additive" traits (Irish, 1998) in their anterior dentitions. Their upper premolars present more simplified occlusal surfaces than $H$. heidelbergensis and $H$. neanderthalensis, and they also present a characteristic bulging on their buccal aspects, which is more pronounced in the $\mathrm{P}^{3} \mathrm{~s}$, and a trait they have in common with Middle Pleistocene groups and $H$. neanderthalensis (Gómez-Robles et al., 2011). The $\mathbf{M}^{1}$ s present a characteristic conformation, with a bulging hypocone protruding in a rhomboidal contour (Bailey, 2004; Gómez-Robles et al., 2007). This conformation is typical of Neanderthals and can be traced back to the Early Pleistocene specimens from Europe (Gómez-Robles et al., 2007, 2011b). While the hypocone is large in the $\mathbf{M}^{1} \mathrm{~s}$, this cusp is absent or only expressed as a vestige in $33.4 \%$ and $95.2 \%$ of the $\mathrm{SH} \mathrm{M}^{2} \mathrm{~s}$ and $\mathrm{M}^{3} \mathrm{~s}$, respectively. This degree of reduction falls within the range of variation of modern populations (see also Bermúdez de Castro, 1987). Lower incisors present moderate labial convexity and lower canines present an incisor-like aspect, with variable degrees of shovel shape and a smooth lingual surface. The $\mathrm{P}_{3} \mathrm{~S}$ present a symmetrical contour where the talonid is extremely reduced or even absent, and the occlusal polygon is very small and located near the lingual border of the crown. In addition, the metaconid is small, centered, and globular, resembling a canine in its conformation.
This shape is typical of the Neanderthal lineage but more pronounced in the SH sample than in other Middle Pleistocene groups such as Arago or even some classic Neanderthals such as some from Krapina and Le Moustier (Gómez-Robles et al., 2008; Gómez-Robles, 2010). The $\mathrm{P}_{4}$ s present an asymmetrical contour, a mesially displaced metaconid, and accessory lingual cusps in association with a continuous transverse ridge. This morphology is typical of $H$. neanderthalensis (Patte, 1959; Genet-Varcin, 1962; Bailey, 2002a,b; Bailey and Lynch, 2005; Martinón-Torres et al., 2006), and, together with an exocentric and reduced occlusal polygon, we can find it in European Early Pleistocene groups (Martinón-Torres et al., 2006). The hypoconulid is reduced (grade $\leq 3)$ in $9.5 \%, 33.4 \%$, and $34.8 \%$ of the $\mathrm{SH} \mathrm{M}_{1} \mathrm{~s}, \mathrm{M}_{2} \mathrm{~s}$, and $\mathrm{M}_{3} \mathrm{~S}$, respectively. This is an exceptional circumstance in a Middle Pleistocene European population (Bermúdez de Castro, 1987; Bermúdez de Castro and Nicolás, 1995; Martinón-Torres, 2006) and could reflect a particularity of this population. In $44.4 \%, 59.1 \%$, and $76.5 \%$ of the SH $M_{1} s, M_{2} s$, and $M_{3} s$, respectively, we see a type of groove pattern different from the dryopithecine-like pattern, a feature related to the dentognathic reduction ascertained in Pleistocene Eurasian populations (Martinón-Torres et al., 2007a), although more pronounced in the $\mathrm{SH}$ group than in $H$. neanderthalensis or $H$. heidelbergensis. The concomitant expression of a deep pit-like anterior fovea and a continuous middle trigonid crest occurs in $85 \%$ of $\mathrm{M}_{1} \mathrm{~S}, 71.4 \%$ of $\mathrm{M}_{2} \mathrm{~S}$, and $57 \%$ of $\mathrm{M}_{3} \mathrm{~S}$, which is similar to the $H$. neanderthalensis condition (Zubov, 1992a,b; Bailey, 2002a,b; Martinón-Torres et al., 2007a,b; Bailey et al., 2011).

With the exception of milder forms of $\mathrm{I}^{1}$ shovel shape and tuberculum dentale, all the traits and conformations described for the SH dentitions are considered typical of $H$. neanderthalensis (Bermúdez de Castro, 1988, 1993; Martinón-Torres, 2006; Martinón-Torres et al., 2007a,b; Gómez-Robles et al., 2007, 2008, 2011; Gómez-Robles, 2010). Although the highest degrees of expression of those particular traits are absent in the SH sample, $H$. neanderthalensis variability also covers $\mathrm{I}^{1} \mathrm{~s}$ with less pronounced shoveling and tuberculum dentale. In general, SH hominins present dentitions that are as "Neanderthal" as those recovered from Krapina or Hortus, and whose larger sample size allow us to consider population variability. With the exceptions mentioned above, the SH hominins show similar or more pronounced degrees and frequencies than $H$. heidelbergensis and even $H$. neanderthalensis for some of the dental traits (this study; see also Gómez-Robles et al., 2008, 2011; Gómez-Robles, 2010). The highly derived state of the Sima de los Huesos dentition cannot be compared to the degree of "neanderthalization" of other penecontemporaneous populations (Tattersall, 2007, 2009; Bermúdez de Castro et al., 2009; Stringer, 2009; Dennell et al., 2010) such as Arago (ca. 350-400 ka; Falguères et al., 2004) or Mauer (ca. 600 ka; Wagner et al., 2010). Although the last age estimate obtained for the Atapuerca-SH site (Bischoff et al., 2007) seems to be controversial for some researchers as it is difficult to accommodate in their "neanderthalization models" (see Stringer, 2009; Endicott et al., 2010), this date has not yet been formally contested anywhere. Even with more conservative estimates for the age of the $\mathrm{SH}$ hominins (>350 ka; Bischoff et al., 2003), they would still be morphologically "more Neanderthal" than the Arago teeth (Bermúdez de Castro et al., 2003a; Gómez-Robles et al., 2007, 2008), and even more than some Neanderthal populations such as Krapina and Hortus, as the SH premolars and upper molars fall in the extreme of the gradient of the Neanderthal variation (Gómez-Robles et al., 2007, 2008, 2011; Gómez-Robles, 2010).

According to Arsuaga et al. (1997c), Middle Pleistocene hominins from Europe and Neanderthals represent the same evolutionary species and could be understood as a chronospecies. With the 
caution of not having a reliable and precise chronological framework for each pre-Neanderthal, it seems that the combination of primitive and derived traits of Middle Pleistocene populations cannot be ordered into a chronological sequence (Bermúdez de Castro et al., 2009; this study). This circumstance is difficult to conciliate with the "accretion model" formulated to explain the emergence of the Neanderthal lineage (Hublin, 1996). According to this model, Neanderthal distinctions would accumulate gradually in populations that became isolated due to glacial conditions, so that earlier populations should be "less Neanderthal" than later populations (Hublin, 1996; Harvati et al., 2010). However, this model was proposed without considering the dental evidence that, until recently, was very scarce for the Middle Pleistocene. Other late Middle Pleistocene specimens from Europe such as the Pontnewydd teeth (ca. $200 \mathrm{ka}$ ) are characterized as being similar to those of the Upper Pleistocene Neanderthals (Green, 1983, 1985; Green et al., 1989) and the much earlier SH teeth (Martinón-Torres, 2006; Gómez-Robles et al., 2007), and, in some tooth classes such as molars and premolars, SH hominins show even more derived conformations than classic Neanderthals such as Krapina, Hortus, and Le Moustier (see Gómez-Robles et al., 2007, 2008, 2011; GómezRobles, 2010). However, it is also important to remember that not all the skeletal parts show the same degree of neanderthalization of the dentition, and these differences were the reason behind excluding the SH fossils from $H$. neanderthalensis (Arsuaga et al., 1991, 1993, 1997c). In contrast, the unclear boundary between the preNeanderthal and Neanderthal populations has led some researchers to suggest that all the Middle and Upper Pleistocene populations showing Neanderthal traits should be included in the H. neanderthalensis lineage (Hublin, 2009).

Unfortunately, the hominin fossil record for the European Middle Pleistocene, aside from the SH sample, is scarce, and we do not really know the true morphological variability of H. heidelbergensis dentitions. Although this species generally overlaps with Neanderthals in its morphology, there are more specimens from the $H$. heidelbergensis sample than from the SH group that fall outside of Neanderthal range of variability (see MDS analyses in SOM), particularly those from Mauer and Arago. In contrast, we do know the variability of the SH group and we can state that the SH hominins are similar to Neanderthals not only "on average" (based on the frequencies of trait expressions) but also individually, as almost all of the specimens, with isolated exceptions, fall within the H. neanderthalensis range of variation (see MDS analyses in SOM).

Hopefully, future hominin discoveries from the Middle Pleistocene of Europe will contribute the necessary evidence to allow a balanced comparison of the degree of neanderthalization between the SH populations and the remaining Middle Pleistocene groups. Until then, we believe that the available evidence compels us to at least explore alternative models to the "accretion model" in order to better understand the emergence of the Neanderthal lineage. Complex demographic patterns driven by the climatic conditions and implying extinctions, recolonizations (Hublin and Roebroeks, 2009), and mixing between demes with different degrees of "neanderthalization" (for a more detailed explanation, see Dennell et al., 2010) could explain the variability of the Middle Pleistocene groups and the apparently non-lineal evolution toward H. neanderthalensis.

Alternatively, the highly derived and Neanderthal character of the Sima de los Huesos dentitions, if we accept the early Middle Pleistocene date attributed to the site, might suggest that SH has played a major role as a core or source population for the Neanderthal gene pool, particularly in the frame of an evolutionary scenario with common bottlenecks, as has been suggested for this lineage (see Hublin, 1996, 2009; Hublin and Roebroeks, 2009). In this scenario, Neanderthals would be a terminal species belonging to a wider European hominin radiation (Schwartz and Tattersall, 2005), and SH (but not other Middle Pleistocene European populations) would be at the root of the classic Neanderthal group.

Otherwise, it could be that the SH hominins represent a different lineage that has evolved in isolation due to hard climatic conditions (see Dennell et al., 2010). Thus, some of their derived morphologies, and the marked reduction of the size of their posterior teeth, could be a particularity of this population (Bermúdez de Castro and Nicolás, 1995). In this framework, and according to some authors (Tattersall and Schwartz, 2006, 2009; Tattersall, 2007, 2009, 2011; Arsuaga, 2009), the allocation of SH in the $H$. heidelbergensis taxon might be reconsidered. The SH hypodigm presents more clear Neanderthal affinities, not only in the dentition, but also in postcranial (Carretero et al., 1997, 2009; Gómez-Olivencia et al., 2007), mandibular (Rosas, 2001) and upper facial morphology (Arsuaga et al., 1997c; Bermúdez de Castro et al., 2009; Tattersall, 2011), than the specimens that are usually included in the $H$. heidelbergensis clade (e.g., Mauer and Arago). However, SH hominins also hold enough differences to prevent their allocation in $H$. neanderthalensis (Arsuaga et al., 1997c; Tattersall, 2011). In this line of reasoning, SH might represent a distinct hominin lineage that is related to Neanderthals, but also different from them and from other European Middle Pleistocene populations. If this were the case, we might be observing the coexistence of two or more hominin lineages during the Middle Pleistocene that are represented by the Sima de los Huesos hominins on one hand and more primitive fossils such as Arago or Mauer on the other. Recent findings, such as the retrieval of the DNA mitochondrial sequence from Denisova (Krause et al., 2010), the new Middle Pleistocene data for the Ceprano calvaria (Manzi et al., 2010), or the finding of an Upper Pleistocene mandible from Mala Balanica (Serbia) without clear Neanderthal traits (Roksandic et al., 2011) might support the possibility of complex evolutionary scenarios with persistence and temporal coexistence of different hominin lineages.

Paradoxically, the Mauer mandible has Neanderthal dental proportions (Rosas and Bermúdez de Castro, 1998) but it lacks some distinctive morphological traits of the European Middle Pleistocene population, such as the typical combination of the middle trigonid crest and a deep, pit-like anterior fovea (Martinón-Torres, 2006; Bailey et al., 2011), or the typical Neanderthal lower second premolars with a reduced exocentric occlusal polygon (MartinónTorres et al., 2006). In this context, we suggest that a revision of the $H$. heidelbergensis taxon would be necessary, due to the lack of consensus among scholars about its taxonomic distinctiveness and its phylogenetic position (see Stringer, 1985; Arsuaga et al., 1997c; Tattersall and Schwartz, 1999; Hublin, 2009). In addition, the Mauer mandible appears as a "problematic" holotype, as it is difficult to link it with other European Middle Pleistocene specimens, mainly characterized by their Neanderthal apomorphies. In other words, Mauer would not be "representative" of the specimens usually assigned to $H$. heidelbergensis.

Finally, it seems clear that $H$. neanderthalensis and $H$. sapiens lineages present different and distinguishable dental patterns. Although our $H$. sapiens sample is biased toward Europe, key differences remain when considering the dental patterns of other modern populations beyond the ones studied here (e.g., Scott and Turner, 1997; Bailey, 2002b; Irish and Guatelli-Steinberg, 2003). However, these differences appear less pronounced between our $H$. heidelbergensis and early $H$. sapiens samples, as stressed by the results of the MMD analysis. Admittedly, both of our $H$. heidelbergensis and fossil $H$. sapiens samples are small, and cover a wider and more heterogeneous geographic and chronological range, and this circumstance obliges us to read this result with 
caution. In addition, it may mean that both populations, at the root of the $H$. neanderthalensis and $H$. sapiens lineages, present less defined (specialized) forms. Indeed, the early $H$. sapiens hominins from Qafzeh display some morphologies considered to be typical of Neanderthals, such as an incipient triangular shovel shape and pronounced incisor labial convexity. Interestingly, Qafzeh fossils also have a perikymata distribution pattern more like that of $H$. erectus and $H$. neanderthalensis (Guatelli-Steinberg and Reid, 2010) than like modern $H$. sapiens. There are two alternative interpretations for the expression of "Neanderthal" traits in this $H$. sapiens population. It could indicate that those traits are indeed synapomorphies inherited from a hypothetical last common ancestor, and that those traits would eventually be lost in $\mathrm{H}$. sapiens while they became typical of $H$. neanderthalensis. In this context, many of the "typical Neanderthal" traits would not be Neanderthal apomorphies but distinctive combinations of primitive features (see also Patte, 1962; Franciscus and Trinkaus, 1995; Bailey, 2002a,b; Martinón-Torres et al., 2006) present in the hominin record prior to the Neanderthal and $H$. sapiens divergence. Alternatively, the $Q$ af zeh hominins could be the result of a hybridization process between early $H$. sapiens and $H$. neanderthalensis in the Middle East, as it has been already posited by the archaeological and molecular data (Bar-Yosef, 1994; Green et al., 2010). However, to address this question it would be desirable to conduct a cladistic rather than a phenetic approach that includes an enlarged comparative sample incorporating earlier hominin species.

\section{Acknowledgments}

We thank all members of the Atapuerca research team, in particular those who excavate the Sima de los Huesos site, for their arduous and dedicated work. We also acknowledge several people for providing access to the studied material and their helpful assistance when examining it: C. Bernis and J. Rascón (Universidad Autónoma de Madrid, Madrid, Spain), A. Pérez-Pérez, J. Galbany, F. Estebaranz, and L. M. Martínez (Universitat de Barcelona, Barcelona, Spain), J. Svoboda (Institute of Archaeology, Paleolithic and Paleoethnology Research Center, Dolní Věstonice, Czech Republic), E. Baquedano (Museo Arqueológico Regional de la Comunidad, Madrid, Spain), J.E. Egocheaga (Universidad de Oviedo, Oviedo, Spain), I. Tattersall, K. Mowbray, and G. Sawyer (American Museum of Natural History, New York), E. Cunha (Universidad de Coimbra, Coimbra, Portugal), H. de Lumley, M.-A. de Lumley, and A. Vialet (Institut de Paléontologie Humaine, Paris, France), C. Stringer (Museum of Natural History, London), and P. Tassy (Muséum National d'Histoire Naturelle, Paris, France). We are also grateful to A. Muela and S. Sarmiento for technical support and photographing part of the sample; P. Fernández Colón and E. Lacasa Marquina from the Restoration and Conservation Laboratory of the CENIEH, and M.C. Ortega from Centro de Evolución y Comportamiento Humanos (ISCIII, Madrid) for the restoration and conservation of the $\mathrm{SH}$ collection; A. Gracia Téllez from Alcalá de Henares University for everything M.M.-T. has learned from her exemplary dedication to these fossils; L. Martín-Francés, M.J. Sier, and M. Sier-Martinón for their valuable assistance in many aspects of this manuscript preparation; J.A. Martín, E. Bruner, J.M. de la Cuétara from the CENIEH and, specially, C. Orton from the University College of London, for their priceless assistance and suggestions in the statistical analysis. Any error is solely the responsibility of the authors. We thank S. Bailey for providing us with the MMD software and instructions. We also acknowledge the anonymous reviewers, the Editor S. Leigh, the Associate Editor D. Guatelli-Steinberg, and G.R. Scott, for valuable revisions and enlightening comments that have definitely helped to improve the manuscript. We also thank J. Blumenf eld and $\mathrm{N}$. Uhl for their careful work in editing the manuscript. This research was supported with funding from the Dirección General de Investigación of the Spanish Ministerio de Educación y Ciencia (MEC), Project $\mathbf{N}^{\circ}$ CGI.2009-12703-C03-01, 02, and 03 and Acción Integrada España Francia HF2007-0115, and Grupo de Excelencia GR-249 from the Conse jería de Universidades e Investigación of the Junta de Castilla y León. Fieldwork at Atapuerca is supported by the Consejería de Cultura y Turismo of the Junta de Castilla y León and the Fundación Atapuerca. L.P.-S. has the benefit of a predoctoral grant of the Fundación Atapuerca and A.G.R. had the support of a predoctoral FPU grant of the Spanish Ministerio de Ciencia e Innovación.

\section{Appendix. Supplementary material}

The supplementary data associated with this article can be found in the on-line version at doi:10.1016/j.jhevol.2011.08.007.

\section{References}

Arsuaga, J.L., 2009. The origin of the Neanderthals. In: Finlayson, C. (Ed.), Calpe Conference 2009, Human Evolution: 150 Years After Darwin. The Gibraltar Museum, Gibraltar, p. 56

Arsuaga, J.L., Carretero, J.M., Lorenzo, C., Gracia, A., Martínez, I., Bermûdez de Castro, J.M., Carbonell, E., 1997a. Size variation in Middle Pleistocene humans. Science 277, 1086-1088.

Arsuaga, J.L., Carretero, J.M., Martínez, I., Gracia, A., 1991. Cranial remains and long bones from Atapuerca/Ibeas (Spain). J. Hum. Evol. 20, 191-230.

Arsuaga, J.L., Martínez, I., Gracia, A., Carretero, J.M., Carbonell, E., 1993. Three new human skulls from the Sima de los Huesos Middle Pleistocene site in Sierra de Atapuerca, Spain. Nature 362, 534-537.

Arsuaga, J.L., Martinez, I., Gracia, A., Carretero, J.M., Lorenzo, C., García, N. Ortega, A.I., 1997b. Sima de los Huesos (Sierra de Atapuerca, Spain): the site. J. Hum. Evol. 33, 109-127.

Arsuaga, J.L., Martínez, I., Gracia, A., Lorenzo, C., 1997c. The Sima de los Huesos crania (Sierra de Atapuerca, Spain): a comparative study. J. Hum. Evol. 33, 219-281.

Bailey, S.E., 2002a. A closer look at Neanderthal postcanine dental morphology: the mandibular dentition. Anat. Rec. 269, 148-156.

Bailey, S.E., 2002b. Neandertal dental morphology: implications for modern human origins. Ph.D. Dissertation, Arizona State University.

Bailey, S.E., 2004. A morphometric analysis of maxillary molar crowns of MiddleLate Pleistocene hominins. J. Hum. Evol. 47, 183-198.

Bailey, S.E., Lynch, J.M., 2005. Diagnostic differences in mandibular P4 shape between Neandertals and anatomically modern humans. Am. J. Phys. Anthropol. 126, 268-277.

Bailey, S.E., Weaver, T.D., Hublin, J.-J., 2009. Who made the Aurignacian and other early Upper Paleolithic industries? J. Hum. Evol. 57, 11-26.

Bailey, S.E., Skinner, M.M., Hublin, J.-J., 2011. What lies beneath? An evaluation of the mid-trigonid crest dental trait based on both dentine and enamel expression. Am. J. Phys. Anthropol. doi:10.1002/ajpa.21468.

Bar-Yosef, -. 1994. The contributions of Southwest Asia to the study of the origin of modern humans. In: Nitecki, M.H., Nitecki, D.V. (Eds.), Origins of Anatomically Modern Humans. Plenum Press, New York, pp. 24-66.

Bath-Balogh, M., Selma, K., 1997. Illustrated Dental Embriology, Histology and Anatomy. Elsevier Health Sciences.

Bermîdez de Castro, J.M., 1986. Dental remains from Atpauerca (Spain) I. Metrics. J. Hum. Evol. 15, 265-287.

Bermîdez de Castro, J.M., 1987. Quantitative analysis of the molar-size sequence in human prehistoric populations of the Canary Isles. Arch. Oral Biol. 32, 81-86.

Bermídez de Castro, J.M., 1988. Dental remains from Atapuerca/Ibeas (Spain) II. Morphology. J. Hum. Evol. 17, 279-304.

Bermîdez de Castro, J.M., 1993. The Atapuerca dental remains: new evidence (1987-1991 excavations) and interpretations. J. Hum. Evol. 24, 339-371.

Bermî́dez de Castro, J.M., Díez, C., 1995. Middle Pleistocene mortality pattern and fertility: the case of the Atapuerca hominids (Sima de los Huesos site, Burgos, Spain). Revista Española de Paleontología 10, 259-272.

Bermîdez de Castro, J.M. Nicolás, M.E., 1995. Posterior dental size reduction in hominids: the Atapuerca evidence. Am. J. Phys. Anthropol. 96, 335-356.

Bermîdez de Castro, J.M., Nicolás, M.E., 1997. Paleodemography of the AtapuercaSH Middle Pleistocene hominind sample. J. Hum. Evol. 33, 333-335.

Bermîdez de Castro, J.M., Pérez, P.J., 1995. Enamel hypoplasia in the Middle Pleistocene hominids from Atapuerca (Spain). Am. J. Phys. Anthropol. 96, 301-314.

Bermîdez de Castro, J.M., Rosas, A., 2001. Pattern of dental development in Hominid X:VIII from the Middle Pleistocene Atapuerca-Sima de los Huesos site (Spain). Am. J. Phys. Anthropol. 114, 325-330.

Bermîdez de Castro, J., Bromage, T.G., Jalvo, Y.F., 1988. Buccal striations on fossil human anterior teeth: evidence of handedness in the middle and early Upper Pleistocene. J. Hum. Evol. 17, 403-412. 
Bermîdez de Castro, J.M., Durand, A.I., Ipiña, S.L, 1993. Sexual dimorphism in the human dental sample from the SH site (Sierra de Atapuerca, Spain): a statistical approach. J. Hum. Evol. 24, 43-56.

Bermı̂dez de Castro, J.M., Martinón-Torres, M., Bermı́dez de Castro, A., Muela, A. Sarmiento, S., Lozano, M., 2004a. Paleodemografia del yacimiento del Pleistoceno medio de la Sima de los Huesos (Sierra de Atapuerca, Burgos). In Baquedano, E., Rubio, S. (Eds.), Miscelánea en homenaje a Emiliano Aguirre Museo Arqueológico Regional, Alcalá de Henares, pp. 10-23.

Bermî́dez de Castro, J.M., Martinón-Torres, M., Carbonell, E., Sarmiento, S. Rosas, A., Van der Made, J., Lozano, M., 2004b. The Atapuerca sites and their contribution to the knowledge of human evolution in Europe. Evol. Anthropol. 13, 25-41

Bermîdez de Castro, J.M., Martinón-Torres, M., Gómez-Robles, A. Margvelashvili, A. Sarmiento, S, 2009. Gli omini di Atapuerca-Sima de los Huesos e le origini dei Neandertaliani. In: Facchini, F., Belcastro, M.G. (Eds.), La Lunga Storia di Neandertal. Editoriale Jaca Book Spa, Milan, pp. 61-75.

Bermûdez de Castro, J.M., Martinón-Torres, M., Lozano, M., Sarmiento, S., Muela, A 2004c. Paleodemography of the Atapuerca-Sima de los Huesos hominin sample: a revision and new approaches to the paleodemography of the Euro pean Middle Pleistocene population. J. Anthropol. Res. 60, 5-26.

Bermûdez de Castro, J.M., Martinón-Torres, M., Sarmiento, S., Lozano, M., 2003a. Gran Dolina-TD6 versus Sima de los Huesos dental samples from Atapuerca: evidence of discontinuity in the European Pleistocene population? J. Archaeol Sci. 30, 1421-1428.

Bermûdez de Castro, J.M., Martinón-Torres, M., Sarmiento, S., Lozano, M. Arsuaga, J.L, Carbonell, E., 2003b. Rates of anterior tooth wear in Middle Pleistocene hominins from Sima de los Huesos (Sierra de Atapuerca, Spain) Proc. Natl. Acad. Sci. USA 100, 11992-11996.

Bermîdez de Castro, J.M., Ramírez, F., Martinón-Torres, M., Sarmiento, S., Rosas, A. 2003c. Patterns of dental development in lower and middle Pleistocene hominids from Atapuerca. In: Thompson, J.L, Krovitz, G.E., Nelson, A.J. (Eds.), Patterns of Growth and Development in the Genus Homo. Cambridge University Press, Cambridge, pp. 246-270.

Bermûdez de Castro, J.M., Sarmiento, S., Cunha, E., Rosas, A., Bastir, M., 2001. Denta size variation in the Atapuerca-SH Middle Pleistocene hominids. J. Hum. Evol 41, 195-209.

Berry, A., Berry, R, 1967. The antrhopological value of minor variants of the dental crown. Am. J. Phys. Anthropol. 45, 257-268.

Bischoff, J.L., Fitzparick, J.A., León, L., Arusaga, J.L., Falguères, C., Bahain, J.J. Bullen, T., 1997. Geology and preliminary dating of the Sima de los Huesos Chamber, Cueva Mayor of the Sierra de Atapuerca, Burgos. Spain. J. Hum Evol 33, 109-127.

Bischoff, J.L., Shamp, D.D., Aramburu, A., Arsuaga, J.L., Carbonell, E., Bermudez de Castro, J.M., 2003. The Sima de los Huesos hominids date to beyond U/Th equilibrium ( $>350 \mathrm{kyr}$ ) and perhaps to $400-500 \mathrm{kyr}$ : new radiometric dates. J. Arch. Sci. 30, 275-280

Bischoff, J.L., Williams, RW., Rosenbauer, R.J., Aramburu, A., Arsuaga, J.L., García, N. Cuenca-Bescós, G., 2007. High-resolution U-series dates from the Sima de los Huesos hominids yields 600 kyrs: implications for the evolution of the early Neanderthal lineage. J. Archaeol. Sci. 34, 763-770.

Boule, M., 1923. Fossil Man. Oliver and Boyd, London.

Boule, M., Vallois, H., 1957. Fossil Men. Dryden Press, New York.

Carbonell, E., Mosquera, M., Ollé, A., Rodríguez, X.P., Sala, R, Vergès, J.M. Arsuaga, J.L., Bermîdez de Castro, J.M., 2003. Les premiers comportement funéraires auraient-ils pris place à Atapuerca, il y a 350000 ans? L'Anthropologie 107, 1-14.

Carlsen, ๑., 1987. Dental Morphology. Munksgaard, Copenhagen.

Carretero, J.M., Arsuaga, J.L., Lorenzo, C., 1997. Clavicles, scapulae and humeri from the Sima de los Huesos site (Sierra de Atapuerca, Spain). J. Hum Evol 33, 357-408.

Carretero, J.M., Haile-Selassie, Y., Rodríguez, L., Arsuaga, J.L., 2009. A partial distal humerus from middle Pleistocene deposits at Bodo, middle Awash, Ethiopia. Anthropol. Sci. 117, 19-31.

Crummett, T., 1995. The three dimensions of shovel-shaping. In: Moggi-Cecchi, J (Ed.), Aspects of Dental Biology: Palaeontology, Anthropology and Evolution International Institute for the Study of Man, Florence, pp. 305-313.

Cunha, E., Ramirez Rozzi, F., Bermîdez de Castro, J.M., Martinón-Torres, M Wasterlain, S.N., Sarmiento, S., 2004. Enamel hypoplasias and physiological stress in the Sima de los Huesos Middle Pleistocene hominins. Am. J. Phys Anthropol. 125, 220-231.

Dahlberg, A.A., 1945. The changing dentition of man. J. Am. Dent Assoc. 34 $676-690$.

Dean, D., Hublin, J.-J., Holloway, R, Ziegler, R, 1998. On the phylogenetic position of the pre-Neandertal specimen from Reilingen, Germany. J. Hum. Evol. 34 485-508.

Dennell, RW., Martinón-Torres, M., Bermûdez de Castro, J.M., 2010. Hominin variability, climatic instability and population demography in Middle Pleistocene Europe. Quat Sci. Rev.. doi: 10.1016/j.quascirev.2009.11.027

Endicott, P., Ho, S.Y.W., Stringer, C.B., 2010. Using genetic evidence to evaluate four palaeoanthropological hypotheses for the timing of Neanderthal and modern human origins. J. Hum Evol. 59, 87-95.

Falguères, C., Yokoyama, Y., Shen, G., Bischoff, J.L., Ku, T.-L, de Lumley, H., 2004 New U-series dates at the Caune de I'Arago, France. J. Archaeol. Sci. 31 941-952.

Franciscus, R.,. Trinkaus, E., 1995. Determinants of retromolar space presence in Pleistocene Homo mandibles. J. Hum. Evol. 28, 577-595. arcía, N., Arsuaga, J.L, 2010. The Sima de los Huesos (Burgos, northern Spain): palaeoenvironment and habitats of Homo heidelbergensis during the Middle Pleistocene. Quat. Sci. Rev. doi:10.1016/j.quascirev.2010.11.008.

Genet-Varcin, E., 1962. Évolution de la couronne de la seconde prémolaire inférieure chez les hominidés. Ann. Paléontol. (Vert.) XLVIII, 59-81.

Gómez-livencia, A., 2009. Estudios paleobiológicos sobre la columna vertebral y la caja torácica de los humanos fósiles del pleistoceno, con especial referencia a los fósiles de la Sierra de Atapuerca. Ph.D. Dissertation, University of Burgos.

Gómez-Olivencia, A., Carretero, J.M., Arsuaga, J.L., Rodríguez-García, L., GarcíaGonzález, R. Martínez, I., 2007. Metric and morphological study of the upper cervical spine from the Sima de los Huesos site (Sierra de Atapuerca, Burgos, Spain). J. Hum. Evol. 53, 6-25.

Gómez-Robles, A, 2010. Análisis de la forma dental en la filogenia humana. Tendencias y modelos evolutivos basados en métodos de morfomería geométrica. Ph.D. Dissertation, University of Granada.

Gómez-Robles, A., Bermîdez de Castro, J.M., Martinón-Torres, M., Prado-Simón, L, 2011b. Crown size and cusp proportions in $H$. antecessor upper first molars. A comment on Quam et al. 2009. J. Anat. 218, 258-262.

Gómez-Robles, A., Martinón-Torres, M., Bermûdez de Castro, J.M., Margvelashvili, A., Bastir, M., Arsuaga, J.L, Pérez-Pérez, A., Estebaranz, F., Martinez, L.M., 2007. A geomeric morphometric analysis of hominin upper first molar shape. J. Hum. Evol. 53, 272-285.

Gómez-Robles, A., Martinón-Torres, M., Bermûdez de Castro, J.M., Prado, L. Sarmiento, S., Arsuaga, J.L., 2008. Geomerric morphometric analysis of the crown morphology of the lower first premolar of hominins, with special attention to Pleistocene Homo. J. Hum. Evol. 55, 627-638.

Gómez-Robles, A., Martinón-Torres, M., Bermı̂dez de Castro, J.M., Prado-Simón, L. Arsuaga, J.L., 2011. A geometric morphometric analysis of hominin upper premolars. Shape variation and morphological integration. J. Hum. Evol. 61 (6), $688-702$.

Gómez-Robles, A., Olejniczak, A.J., Martinón-Torres, M., Prado-Simón, L., Bermûdez de Castro, J.M., 2011a. Evolutionary novelties and losses in geometric morphometrics: a practical approach through hominin molar morphology. Evolution. doi: 10.1111/j.1558-5646.2011.01244.x

Green, H.S., 1983. La Grotte de Pontnewydd, Pays de Galles (Grande-Bretagne): un site du Paléolithique inférieur avec des restes humains probablement Néandertaliens archaïques. L'Anthropologie 97, 417-419.

Green, H.S., 1985. Ponmewydd Cave: a Lower Palaeolithic Hominid Site in Wales. The First Report National Museum of Wales, Quaternary Studies Monographs 1 , Cardiff.

Green, H.S., Bevins, R.E., Bull, P.A., Currant, A.P., Debenham, N.C., Embleton, C. Ivanovich, M., Livingston, H., Rae, A.M., Schwarcz, H.P., Sringer, C.B., 1989. Le site Acheuléen de la Grotte de Pontnewydd, Pays de Galles: géomorphologie, stratigraphie, chronologie, faune, hominidés fóssiles, géologie et industrie lithique dans le context Paléoécologique. L'Anthropologie 93, 15-52.

Green, RE., Krause, J., Briggs, A.W., Maricic, T., Stenzel, U., Kircher, M., Patterson, N., Li, H., Zhai, W., Fritz, M.H.-Y., Hansen, N.F., Durand, E.Y., Malaspinas, A.-S., Jensen, J.D., Marques-Bonet, T., Alkan, C., Prüfer, K., Meyer, M., Burbano, H.A., Good, J.M., Schultz, R, Aximu-Petri, A., Butthof, A., Höber, B., Höffner, B., Siegemund, M., Weihmann, A., Nusbaum, C., Lander, E.S., Russ, C., Novod, N., Affourtit, J., Egholm, M., Verna, C., Rudan, P., Brajkovic, D., Kucan, Z., Gušic, I., Doronichev, V.B., Golovanova, L.V., Lalueza-Fox, C., de la Rasilla, M., Fortea, J., Rosas, A., Schmitz, RW., Johnson, P.L.F., Eichler, E.E., Falush, D., Birney, E., Mullikin, J.C., Slatkin, M., Nielsen, R, Kelso, J., Lachmann, M., Reich, D., Pããbo, S., 2010. A draft sequence of the Neandertal genome. Science 328, 710-722.

Guatelli-Steinberg, D., Reid, D.J., 2010. The distribution of perikymata on Qafzeh anterior teeth. Am. J. Phys. Anthropol. 141, 152-157.

Hammer, ๑., Harper, D.A.T., Ryan, P.D., 2001. PAST: Paleontological Statistics Software Package for Education and Data Analysis. Palaeontol. Electronica 4, 9.

Harvati, K., Hublin, J.-J., Gunz, P., 2010. Evolution of middle-late Pleistocene human cranio-facial form: a 3-D approach. J. Hum. Evol. 59, 445-464

Hillson, S., 1986. Teeth. Cambridge University Press, Cambridge.

Hrdlička, A., 1924. New data on the teeth of early man and certain fossil European apes. Am. J. Phys. Anthropol. 7, 109-132.

Hlusko, L. J., 2003. Protostylid variationin Australo pithecus. J. Hum. Evol. 46, 579-594.

Hublin, J.-J., 1982. Les ante néandertaliens: présapiens ou prénéandertaliens? Géobios. Mem. Spec. 6, 345-357.

Hublin, J.-J., 1984. The fossil man from Salzgitter-Lebenstedt (FRG) and its place in the human evolution during the Pleistocene in Europe. Z. Morph. Anthropol. 75, $45-56$.

Hublin, J-J., 1996. The first Europeans. Arch. 49, 36-44.

Hublin, J.-J., 1998. Climate change, paleogeography and the evolution of the Neandertals. In: Akazawa, T., Aoki, K., Bar-Yosef, - (Eds.), Neandertals and Modern Humans in Western Asia. Plenum Publishing. New York, pp. 295-310.

Hublin, J.- J., 2009. The origin of Neanderthals. Proc. NatI. Acad. Sci. USA 106, $16022-16027$

Hublin, J.-J., Roebroeks, W., 2009. Ebb and flow or regional extinctions? on the character of Neandertal occupation of northern environments. C. R. Palevol. 8, 503-509.

Irish, J.D. 1993. Biological affinities of late Pleistocene through modern African Aboriginal populations: the dental evidence. Ph.D. Dissertation, Arizona State University.

Irish, J.D., 1998. Diachronic and synchronic dental trait affinities of Late and PostPleistocene peoples from North Africa. Homo 49, 138-155. 
Irish, J.D. 2010. The mean measure of divergence: its utility in model-free and model-bound analyses relative to Mahalanobis $\mathrm{D}^{2}$ distance for nonmetric traits. Am J. Hum. Biol. 22, 378-395.

Irish, J.D., Guatelli-Steinberg, D., 2003. Ancient teeth and modern human origins: an expanded comparison of African Plio-Pleistocene and recent world dental samples. J. Hum. Evol. 45, 113-144.

Kallay,J., 1963. A radiographic study of the Neanderthal teeth from Krapina Croatia. In: Brothwell, D.R (Ed.), Dental Anhropology. Pergamon Press, exford, pp. 75-86.

Keith, A., 1924. Neanderthal man in Malta. J. R. Anthropol. Inst. 54, 251-274.

Keith, A., 1925. The Antiquity of Man. William and Norgate, Ltd., London.

Klein, RG., 1999. The Human Career. University of Chicago Press, Chicago.

Krause, J., Fu, Q., Good, J.M., Viola, B., Shunkov, M.V., Derevianko, A.P., Pããbo, S., 2010. The complete mitochondrial DNA genome of an unknown hominin from southern Siberia. Nature 464, 894-897.

Kricum, M., Monge, J., Mann, A., FinkeI, G., Lampl, M., Radovcic, J., 1999. The Krapina Hominids: a Radiographic Atlas of the Skeletal Collection. Croatian Natural History Museum, Zagreb.

Krogman, W., 1927. Anthropological aspects of the human teeth and dentition. J. Dent. Res. 7, 1-108.

KruskaI, J.B., Wish, M., 1978. Mulidimensional Scaling. Sage Publications, Beverly Hills, CA.

Kupczik, K., Hublin, J.-J., 2010. Mandibular molar roots morphology in Neanderthals and Late Pleistocene and recent Homo sapiens. J. Hum. Evol. 59, 525-541.

Lebel, S., Trinkaus, E., 2002. Middle Pleistocene human remains from the Bua de I'Aubesier. J. Hum. Evol. 43, 659-685.

Lefêvre, J., 1973. Étude odontologique des hommes de Muge. Bull. Mém. Soc. Anthropol. Paris 12, 301-333.

Lorenzo, C., Carretero, J.M., Arsuaga, J.L., Gracia, A., Martínez, I., 1998. Intrapopulational body size variation and cranial capacity variation in Middle Pleistocene humans: the Sima de los Huesos sample (Sierra de Atapuerca, Spain). Am. J. Phys. Anthropol. 106, 19-33.

Lozano, M., Bermîdez de Castro, J.M., Carbonell, E., Arsuaga, J.L., 2008. Nonmasticatory uses of anterior teeth of Sima de los Huesos individuals (Sierra de Atapuerca, Spain). J. Hum. Evol. 55, 713-728.

Lozano-Ruiz, M., Bermîdez de Castro, J.M., Martinón-Torres, M., Sarmiento, S., 2004 Cutmarks on fossil human anterior teeth of the Sima de los Huesos site (Atapuerca, Spain). J. Archaeol. Sci. 31, 1127-1135.

de Lumley, M.-A., 1973. Anténéandertaliens et Néandertaliens du bassin Méditerranéen Occidental Européen. Études quaternaires 2. Université de Provence.

Manzi, G., Magri, D., Milli, S., Palombo, M.R, Vasiliki Margari, V., Celiberti, V., Barbieri, M., Barbieri, M., Melis, RT., Rubini, M., Ruffo, M., Saracino, B., Tzedakis, P.C., Zaratini, A., Biddittu, I., 2010. The new chronology of the Ceprano calvarium (Italy). J. Hum. EvoI. 59, 580-585.

Martinón-Torres, M., 2006. Evolución del aparato dental en homínidos: estudio de los dientes humanos del Pleistoceno de la Sierra de Atapuerca (Burgos). Ph.D. Dissertation, Universidad de Santiago de Compostela.

Martinón-Torres, M., Bastir, M., Bermûdez de Casłro, J.M., Gómez, A., Sarmiento, S. Muela, A., Arsuaga, J.L, 2006. Hominin lower second premolar morphology: evolutionary inferences through geomeric morphometric analysis. J. Hum Evol. 50, 523-533.

Martinón-Torres, M., Bermîdez de Castro, J.M., Gómez-Robles, A., Arsuaga, J.L, Carbonell, E., Lordkipanidze, D., Manzi, G., Margvelashvili, A., 2007b. Dental evidence on the hominin dispersals during the Pleistocene. Proc. Natl. Acad. Sci. USA 104, 13279-13282.

Martinón-Torres, M., Bermî̀dez de Castro, J.M., Gómez-Robles, A., Margvelashvili, A., Prado, L., Lordkipanidze, D., Vekua, A., 2008. Dental remains from Dmanisi (Republic of Georgia): morphological analysis and comparative study. J. Hum. Evol. 55, 249-273.

Martinón-Torres, M., Bermîdez de Castro, J., Gómez-Robles, A., Sarmiento, S., Muela, A., Arusaga, J.L., 2007a. Gran Dolina-TD6 and Sima de los Huesos dental samples: preliminary approach to some dental characters of interest for phylogenetic studies. In: Bailey, S.E., Hublin, J.-J. (Eds.), Dental Perspectives on Human Evolution. Springer-Verlag, Berlin, pp. 65-79.

Mizoguchi, Y., 1985. Shovelling: A Statistical Analysis of Its Morphology. University Museum, Tokyo, Bulletin, pp. 26.

Moggi-Cecchi, J., Grine, F.E., Tobias, P.V., 2006. Early hominid dental remains from Members 4 and 5 of the Sterkfontein Formation (1966-1996 excavations): catalogue, individual associations, morphological descriptions and initial metric analysis. J. Hum Evol. 50, 239-328.

Moggi-Cecchi, J., Menter, C., Boccone, S., Keyser, A., 2010. Early hominin dental remains from the Plio-Pleistocene site of Drimolen, South Africa. J. Hum. Evol. $58,374-405$.

Molnar, S., 1971. Human tooth wear, tooth function and cultural variability. Am. J. Phys. Anthropol. 34, 175-190.

Patte, É, 1959. La dentition des Néandertaliens. Ann. Paléontol. (Vert) 45, 221-305

Patte, É., 1962. La dentition des Néanderthaliens. Masson et Cie, Éditerus, Paris, pp. 237-242.

Pérez-Pérez, A., Bermûdez De Castro, J.M., Arsuaga, J.L, 1999. Nonocclusal dental microwear analysis of 300,000-year-old Homo heidelber gensis teeth from Sima de los Huesos (Sierra de Atapuerca, Spain). Am. J. Phys. Anthropol. 108, 433-457.

Pérez-Pérez, A., Espurz, V., Bermûdez de Castro,J.M., de Lumley, M.-A., Turbón, D., 2003. Non-occlusal dental microwear variability in a sample of Middle and Late Pleistocene humanpopulations from Europe and the Near East J. Hum. Evol 44,497-513. am, R Bailey, S.E, Wood, B.A 2009. Evolution of M1 crown size and cusp proportions in the genus Homo. J. Anat. 214, 655-670.

Roksandic, M., Mihailović, D., Mercier, N., Dimitrijević, V., Morley, M.V. Rakočević, Z., Mihailović, B., Guibert, P., Babb, J., 2011. A human mandible (BH-1) from the Pleistocene deposits of Mala Balanica cave (Sicevo Gorge, Niš, Serbia). J. Hum. Evol. 61, 186-196.

Rosas, A., 2001. -ccurrence of Neanderthal features in mandibles from the Atapuerca-SH site. Am. J. Phys. Anthropol. 114, 74-91.

Rosas, A., Bermî̀dez de Castro, J.M., 1998. The Mauer mandible and the evolutionary significance of Homo heidelbergensis. Geobios 31, 687-697.

Rosas, A., Bastir, M., Martinez-Maza, C., Bermûdez de Castro, J.M., 2002. Sexual dimorphism in the Atapuerca-SH hominids: the evidence from the mandibles. J. Hum. Evol. 42, 451-474.

Scott, G.R., Turner II, C.G., 1997. The Anthropology of Modern Human Teeth: Dental Morphology and Its Variation in Recent Human Populations. Cambridge University Press, Cambridge.

Schwartz, J.H., Tattersall, I., 2005. The Human Fossil Record, Volume 4: Craniodental Morphology of Early Hominids (Genera Australopithecus, Paranthropus, Orrorin) and Overview. Wiley-Liss, New York

Sjovold, T., 1973. The occurrence of minor non-metrical variants in the skeleton and their quantitative reament for population comparison. Homo 24, 204-233.

Smith, P., 1989. Dental evidence for phylogenetic relationships of Middle Paleolithic hominids. In: Vandermeersch, B. (Ed.), L'Homme de Nèanderthal. L'extinction, vol. 7. I'Université de Liège, Liège, pp. 111-120.

Stefan, V.H., Trinkaus, E., 1998. Discrete rait and dental morphometric affinities of the Tabun 2 mandible. J. Hum. Evol. 34, 443-468.

Stringer, C.B., 1985. Middle Pleistocene hominid variability and the origin of Late Pleistocene humans. In: Delson, E. (Ed.), Ancestors: The Hard Evidence. Alan R Liss, New York, pp. 289-296.

Stringer, C.B., 1993. Secrets of the pit of the bones. Nature 362, 501-502.

Stringer, C.B., 2009. The origins and evolution of the Neanderthal and modern human clades. In: Finlayson, C. (Ed.), Calpe Conference 2009, Human Evolution: 150 Years After Darwin. The Gibraltar Museum, Gibraltar, pp. 57-58.

Tattersall, I., 2007. Neanderthals, Homo supiens, and the question of species in paleoanthropology. J. Anthropol. Sci. 85, 139-146.

Tattersall, I., 2009. How many lineages of Homo in the middle and late Pleistocene of Europe? In: Finlayson, C. (Ed.), Calpe Conference 2009, Human Evolution: 150 Years After Darwin. The Gibraltar Museum, Gibraltar, pp. 53-54.

Tattersall, I., 2011. Before the Neanderthals: hominid evolution in middle Pleistocene Europe. In: Condemi, S., Weniger, G.-C. (Eds.), Continuity and Discontinuity in the Peopling of Europe. Springer-Verlag, Berlin, pp. 47-53.

Tattersall, I., Schwartz, J.H., 1999. Hominids and hybrids: the place of Neanderthals in human evolution. Proc. NatI. Acad. Sci. USA 96, 7117-7119.

Tattersall, I., Schwartz, J.H., 2006. The distinctiveness and systematic context of Homo neanderthalensis. In: Harvati, K., Harrison, T. (Eds.), Neanderthals Revisited: New Approaches and Perspectives. Springer, Dordrecht, pp. 9-22.

Tattersall, I., Schwartz, J.H., 2009. Evolution of the genus Homo. Ann. Rev. Earth Planet Sci. 37, 67-92.

Tobias, P.V., 1991. Olduvai Gorge. In: The Skulls, Endocasts and Teeth of Homo habilis, vol. 4. Cambridge University Press, Cambridge.

Trinkaus, E., 1983. The Shanidar Neanderthals. Academic Press, New York.

Trinkaus, E., Bailey, S., Zilhão, J., 2001. Upper Paleolithic human remains from the Gruta do Caldeirão, Tomar, Portugal. Revista Portuguesa de Arqueologia 4, 5-17.

Turner II, C.G., 1969. Microevolutionary interpretations from the dentition. Am. J Phys. Anthropol. 30, 421-426.

Turner II, C.G., 1987. Late Pleistocene and Holocene population history of East Asia based on dental variation. Am. J. Phys. Anthropol. 73, 305-321.

Turner II, C.G., NichoI, C.R, Scott, G.R, 1991. Scoring procedures for key morphological traits of the permanent dentition: the Arizona State University dental anthropology system. In: Kelley, M., Larsen, C. (Eds.), Advances in Dental Anthropology. Wiley-Liss, New York, pp. 13-31.

Wagner, G.A., Krbetscheck, M., Degering, D., Bahain, J.-J., Shao, e. Falguères, C. Voinchet, P., Dolo, J.-J., Garcia, T., Rightmire, P., 2010. Radiometric dating of the type-site for Homo heidelbergensis at Mauer. Germany. Proc. Natl. Acad. Sci. USA 107, 19726-19730.

Weidenreich, F., 1937. The Dentition of Sinanthropus Pekinensis: A Comparative -dontography of the Hominids. Palaeontologica Sinica N.S. D1. The Geological Survey of China.

Wood, B.A., Abbot, S.A., 1983. Analysis of the dental morphology of Plio-Pleistocene hominids. I. Mandibular molars: crown area measurements and morphological traits. J. Anat. 136, 197-219.

Wood, B.A., Uytterschaut, $\mathrm{H}, 1987$ Analysis of the dental morphology of PlioPleistocene hominins. Ill. Mandibular premolar crowns. J. Anat. 154, 121-156.

Wood, B., Abbott, S.A., Uytterschaut, H.T., 1988. Analysis of the dental morphology of Plio-Pleistocene hominids IV. Mandibular postcanine root morphology. J. Anat. $156,107-139$.

Wolpoff, M.H., 1971. Metric Trends in Hominid Dental Evolution. Case Western Reserve University. Studies in Anthropology 2

Zilhão, J., 1997. Paleolítico Superior da Estremadura portuguesa. Colibri, Lisboa.

Zubov, A.A., 1992a. The epicristid or middle trigonid crest defined. Dent Anthropol NewsI. 6, 9-10

Zubov, A.A., 1992b. Some dental traits in different evolutionary lines leading to modern man. Dent. Anthropol. Newsl. 6,4-8. 